\title{
Improving Electrical Impedance Tomography Imaging of the Brain
}

\author{
by \\ Mark A. Campbell, BSc, University of Ottawa \\ A thesis submitted to the Faculty of Graduate and Postdoctoral Affairs \\ in partial fulfillment of the requirements for the degree of
}

\author{
Master of Applied Science
}

in

Biomedical Engineering

\author{
Carleton University \\ Ottawa, Ontario
}

(C) 2020

Mark A. Campbell 


\section{Abstract}

Hemorrhagic transformation (HT) is an ischemic stroke complication occurring in 10\% of ischemic stroke patients with infarcts. HT is detected by symptom and CT monitoring, though exposes patients to repeated radiation doses. Symptom monitoring for detecting perioperative ischemia is ineffective in anesthetized patients. A non-ionizing modality for continuous monitoring of cerebral blood volume (CBV) would therefore be valuable. Electrical impedance tomography (EIT) is an imaging technique that reconstructs the internal conductivity distribution of a body non-invasively. EIT can detect changes in CBV, but only hemorrhage, not ischemia, has been detected with EIT. A novel EIT processing method was developed that depicted arterial blood pressure related CBV changes and successfully detected ischemia in swine, as verified by MRI. Software tools and EIT electrode hardware were developed to apply this method to functional imaging studies in humans. Finally, an automated electrode quality and data rejection tool, which simplifies EIT preprocessing, was developed. 


\section{Acknowledgements}

I would like to thank my supervisors Dr. Andy Adler and Dr. Rebecca Thornhill for their support and guidance throughout the making of this work. The feedback from Aaron Hill on the MIT Open-Courseware Python courses taken during the summer before this degree was of tremendous value. I would like to extend my gratitude towards my past supervisor Dr. Georg Northoff and his group, especially Dr. Shankar Tumati and Mehrshad Golesorkhi, for the opportunity to work with their lab and attend their meetings.

I extend my gratitude to Dr. Constantin Trepte's group at UKE Hamburg, for the data used in chapter 3 was collected by his team. I would especially like to thank Dr. Christoph Behem for his continued support and help with accessing the EIT, CT, and MRI files, as well as his contributions to the methods section of the manuscript. The weighted restraint manuscript would not have been possible without Symon Stowe, who designed the protocol. Thanks to Malitela Mapani for her help with the weighted restraint data collection, and to Dr. Jeff Dawson and Dr. Andy Adler for their help with the manuscript. The help of Tarek Harake with the additive manufacturing of electrode prototypes was greatly appreciated.

I would like to thank the members of Dr. Adler's lab: Aaron Hill, Alistair Boyle, Dave Dawson, and Symon Stowe, for the technical discussions, suggestions, 
and friendship. Finally, I would like to send out a special thank you to my fiancée, friends, and family for their continued love and support, and their understanding of my apparent de-materialization during the final writing of this work. 
"If our brains were simple enough for us to understand them, we'd be so simple that we couldn't."

- G.E. Pugh, in The Biological Origin of Human Values, 1978. 


\section{Contents}

1 Introduction 1

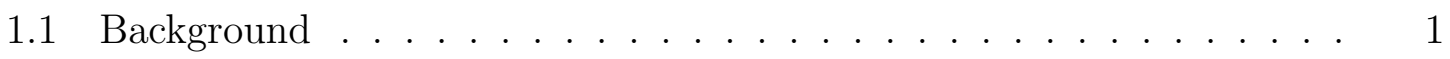

1.2 Goals . . . . . . . . . . . . . . . . . . . . . . 2

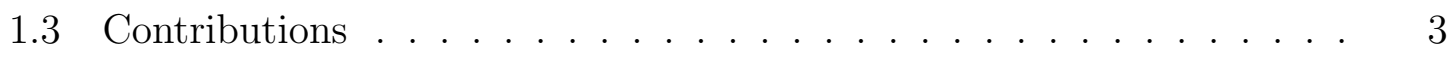

2 Literature Review $\quad 6$

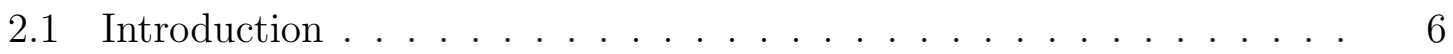

2.2 Electrical Impedance Tomography . . . . . . . . . . . . . . . . 7

2.2 .1 How EIT Works . . . . . . . . . . . . . . . 9

$2.2 .2 \quad$ EIT Image Reconstruction . . . . . . . . . . . . . . . 11

2.3 Sources of EIT Brain Conductivity Changes $\ldots \ldots \ldots \ldots$

2.3 .1 Cerebral Edema . . . . . . . . . . . . . . . . . 16

2.4 Stroke Imaging $\ldots \ldots \ldots \ldots \ldots$

2.4 .1 Stroke Imaging with EIT . . . . . . . . . . . . . . . . . 19

2.5 Functional Neuroimaging . . . . . . . . . . . . . . . . . . 21

2.5.1 The Hemodynamic Response . . . . . . . . . . . . . . . . . 21

2.5.2 Principles of Brain Imaging and Major Modalities . . . . . . 22

2.5.3 Functional Neuroimaging with EIT . . . . . . . . . . . . 24

2.6 Advanced EIT Electrode Arrays for Brain Imaging $\ldots \ldots \ldots$ 
3 Perfusion-Based Methods for Detecting Cerebral Ischemia with Electrical Impedance Tomography $\quad 29$

3.1 Introduction . . . . . . . . . . . . . . . . . . . . 30

3.2 Methods . . . . . . . . . . . . . . . . . . . . . 33

3.2 .1 Ethics . . . . . . . . . . . . . . . . . . . 33

3.2 .2 Study Design . . . . . . . . . . . . . . . . . . . . . 33

3.2.3 Animal Care, Anaesthesia and Surgical Procedures . . . . . 34

3.2 .4 Data Collection . . . . . . . . . . . . . . . . . 35

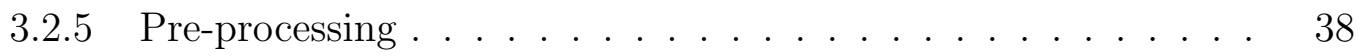

3.3 Results . . . . . . . . . . . . . . . . . . . . . . . . . . 40

3.3 .1 Ensemble Average . . . . . . . . . . . . . . . . 40

3.3 .2 Saline Bolus . . . . . . . . . . . . . . . . . . . . . . 50

3.4 Discussion . . . . . . . . . . . . . . . . . . . . . . 52

4 Towards Measuring Brain Function with Perfusion EIT 56

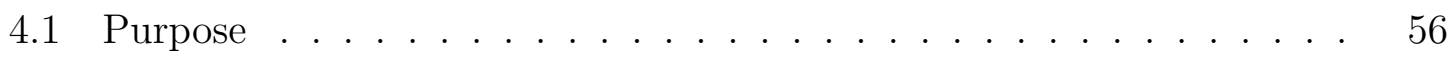

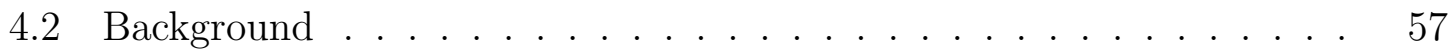

$4.2 .1 \quad$ EIT Electrodes $\ldots \ldots \ldots \ldots \ldots \ldots$

$4.2 .2 \quad$ Existing Headset Prototype . . . . . . . . . . . . . . 59

4.3 Methods . . . . . . . . . . . . . . . . . . . 60

4.3.1 Design and Fabrication of Electrodes for EIT Measurements on Skin with a Thick Hair Covering $\ldots \ldots$. . . . . . 60

4.3.2 Creating a Realistic Head Model from Paired CT-MRI Images 62

4.3.3 Reconstructions From Simulated Data . . . . . . . . . . 65

4.3.4 Protocol Design . . . . . . . . . . . . . . . 66

4.3.5 N-Back Graphical User Interface (GUI) Software . . . . . 67 
4.4 Results . . . . . . . . . . . . . . . . . . . 68

4.4.1 Electrode Tests . . . . . . . . . . . . . . . . 68

4.4 .2 Simulations . . . . . . . . . . . . . . 70

4.5 Discussion . . . . . . . . . . . . . . . . . . 73

4.5.1 Improvements to the electrode array . . . . . . . . . . . . 73

4.5.2 Simulations . . . . . . . . . . . . . . 76

\section{Automated Electrode Quality Assessment and Data Rejection} $\begin{array}{ll}\text { (EQADR) Algorithm } & 78\end{array}$

5.1 Introduction . . . . . . . . . . . . . . . . . . . 78

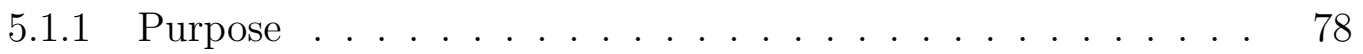

5.1.2 Defining High-Quality EIT Data . . . . . . . . . . . . . 79

5.1.3 Methods of Detecting Faulty Electrodes and "Bad" Measurements 79

5.2 Methods ............................. 82

5.2.1 Identification of Erroneous Measurements . . . . . . . . . . . 82

5.3 Results \& Discussion . . . . . . . . . . . . . . . . . . . . 86

6 Discussion \& Future Work $\quad 90$

6.1 Summary . . . . . . . . . . . . . . . . . . . . 90 90

6.2 Discussion . . . . . . . . . . . . . . . . . . . . . . . . 91

6.2.1 EIT Stroke Methods . . . . . . . . . . . . . . . . . . . . 91

6.2.2 EIT Electrode Design . . . . . . . . . . . . . . . . 91

6.2.3 Realistic Human Head Reconstruction Model and Simulations 92

6.2.4 N-Back Graphical User Interface (GUI) Software . . . . . . . 92

6.2.5 Weighted Restraint . . . . . . . . . . . . . . 93

6.3 Future Work . . . . . . . . . . . . . . . . . . 93

6.4 Conclusion . . . . . . . . . . . . . . . . . . . . . . . 94 
A Using Electrical Impedance Tomography in an Experimental Model of Weighted Restraint

A.1 Abstract . . . . . . . . . . . . . . 96

A.2 Introduction . . . . . . . . . . . . . . . . . 96

A.3 Methods . . . . . . . . . . . . . . . . . . . . . 99

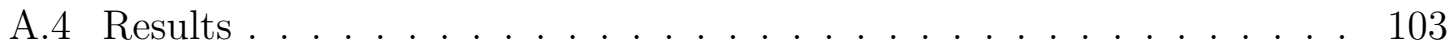

A.5 Discussion . . . . . . . . . . . . . . . . . . . . . . . . 109

B Functional Neuroimaging of the N-back Working Memory Task with 3D Electrical Impedance Tomography REB Protocol Form 


\section{Table of Acronyms}

\begin{tabular}{ll}
\multicolumn{1}{c}{ Acronym } & Details \\
\hline 3 Rs & Replacement, Reduction, and Refinement \\
\hline${ }^{18}$ FDG & 18-FluoroDeoxyGlucose \\
\hline${ }^{18}$ FDG-PET & 18-FluoroDeoxyGlucose Positron-Emission Tomography \\
\hline ABP & Arterial Blood Pressure \\
\hline ADC & Analog to Digital Converter / Apparent Diffusion Coefficient \\
\hline ADP & Adenosine-Diphosphate \\
\hline AM & Additive Manufacturing \\
\hline ARRIVE & Animal Research: Reporting of In Vivo Experiments \\
\hline ASCII & American Standard Code for Information Interchange \\
\hline ATP & Adenosine-Triphosphate \\
\hline BBB & Blood-Brain Barrier \\
\hline BOLD & Blood-Oxygen Level Dependent \\
\hline CBF & Cerebral Blood Flow \\
\hline CBV & Cerebral Blood Volume \\
\hline CSF & Cerebrospinal Fluid \\
\hline $\mathrm{CT}$ & Computed Tomography \\
\hline $\mathrm{C}_{Z}$ & Contact Impedance \\
\hline
\end{tabular}




\begin{tabular}{ll}
\hline $\mathrm{dHb}$ & Deoxy-Hemoglobin \\
\hline DW-MRI & Diffusion-Weighted Magnetic Resonance Imaging \\
\hline ECG & Electrocardiogram \\
\hline EEG & Electroencephalogram \\
\hline EIT & Electrical Impedance Tomography \\
\hline EIT-BI & Electrical Impedance Tomography Brain Imaging \\
\hline EQADR & Electrode Quality Assessment and Data Rejection algorithm \\
\hline fdEIT & Frequency-Difference Electrical Impedance Tomography \\
\hline FELASA & Federation for Laboratory Animal Science Associations \\
\hline FEM & Finite Element Method / Finite Element Model \\
\hline fMRI & Functional Magnetic Resonance Imaging \\
\hline GUI & Graphical User Interface \\
\hline Hb & Hemoglobin \\
\hline HDR & Hemodynamic Response \\
\hline HMPAO & 99m Tc-Hexamethyl Propylene Amine Oxime \\
\hline HT & Hemorrhagic Transformation \\
\hline Iz & Inion \\
\hline mfEIT & Multi-Frequency Electrical Impedance Tomography \\
\hline MRI & Magnetic Resonance Imaging \\
\hline NP & National Institutes of Health \\
\hline PAL & Nanoparticles \\
\hline
\end{tabular}




\begin{tabular}{ll}
\hline PW-MRI & Perfusion-Weighted Magnetic Resonance Imaging \\
\hline rCBV & Relative Cerebral Blood Volume \\
\hline SEM & Scanning Electron Microscopy \\
\hline SNR & Signal-to-Noise Ratio \\
\hline STEM & SenTec EIT Monitor \\
\hline SPECT & Single-Photon Emission Computed Tomography \\
\hline tdEIT & Time-Difference Electrical Impedance Tomography \\
\hline
\end{tabular}




\section{Chapter 1}

\section{Introduction}

\section{$1.1 \quad$ Background}

Stroke is the second largest cause of death worldwide and causes morbidities such as permanent disability in up to $50 \%$ of survivors [1]. Diagnosis and classification of stroke type as either ischemic or hemorrhagic is performed by perfusion CT and CT angiography [2]. MRI may be used to determine the age of the stroke when the time of onset is unknown [3]. The timely and accurate classification of stroke type and age is critical for informing the correct medical intervention and justifies the radiation dose and cost of imaging. Settings in which repeated CT imaging is used to monitor the new occurrence of hemorrhagic transformation (HT) in severe cases of ischemia, or ischemia intraoperatively, can benefit from a non-ionizing continuous monitoring device that increases overall temporal resolution, reduces the cost of imaging, and minimizes the radiation dose to the patient.

Electrical impedance tomography (EIT) is an imaging technique that satisfies the criteria above, being safe, inexpensive, portable, and well-suited to continuous and long-term medical imaging applications. EIT images the distribution of conductivity 
within a body non-invasively from surface measurements and has the potential to image changes in cerebral blood volume (CBV) that occur in hemorrhage and ischemia. The distinct electrical conductivities of various biological tissues, especially blood, can be exploited by EIT to detect changes in CBV. EIT will never replace CT and MR imaging for initial stroke diagnosis and classification but could provide a means of warning medical personnel of stroke onset from intraoperative complications, or from HT at the bedside. If successful, EIT in this context could reduce hospital imaging costs and radiation doses to patients, while improving patient outcomes by decreasing the time to detection of intraoperative stroke and HT.

\subsection{Goals}

The primary goal of this work was to develop perfusion-based methods for detecting changes in CBV that occur in stroke, with a focus on detecting cerebral ischemia in an animal model. Next, this work sought to explore the feasibility of using these perfusion-based methods and EIT hardware to detect the small-scale CBV changes that occur in functional brain activity in a human study. The subsequent goal of this work was then to develop and test novel EIT electrodes capable of attaining adequate contact quality in the presence of thick hair, for use in human EIT brain imaging (EIT-BI). Lastly, a goal of this thesis was to develop an automated EIT electrode quality assessment and data rejection tool, motivated by a need for clean and consistent measurement rejection in data from the stroke study. Given the sensitivity of EIT to noise, it is beneficial to remove noisy measurements to ensure quality images and results, especially for EIT-BI. Data rejection a tedious process that requires significant technical experience. An automated tool for EIT data cleaning would therefore expedite analysis and increase the overall accessibility of EIT. 


\subsection{Contributions}

Two novel methods for detecting changes in relative CBV (rCBV) in an animal model of ischemic stroke were developed. The first method used a saline bolus injected into the circulation to act as an EIT contrast agent. Saline injections have been used previously to accurately depict perfusion changes in the lung but were not successful in the current study. Though the method itself was not novel, this was the first use of a saline bolus injection for EIT-BI using surface, rather than cortical electrodes. The second method developed used the direct relationship between arterial blood pressure and volume, which is a maximum in the systolic phase, to produce a sequence of EIT images averaged over multiple cardiac cycles to depict arterial pressure-related rCBV changes, while improving the SNR. This method accurately localized ischemic regions in $4 / 5$ animals, as evidenced by MRI.

The success of the ensemble method was important not only because it was the first study in which ischemia was reliably detected with EIT, but because it showed that injection of saline, which is an invasive procedure, is not required to depict perfusion with EIT. Instead, non-invasive cardiac measures such as ECG or PPG could be used with this method to depict perfusion with EIT.

To apply the ensemble technique to human functional imaging studies, an elec-

trode headset and realistic reconstruction model were developed. Normal ECG-type EIT electrodes cannot be used over the thick hair covering of the head and EIT-BI is in its infancy, meaning that EIT-specific electrode headsets are not commercially available. Therefore, a novel electrode, incorporating an array of 19 P75-D spring contact probes in a rotating housing, that permitted scalp abrasion, was designed, manufactured, and tested. Though the full electrode headset was not completed at the time of writing, a description of its intended design was provided. Secondly, a 
realistic human head reconstruction model with 3D electrode configuration matching that of the headset design was produced from paired CT and MRI images. The quantity and locations of electrodes are customizable, allowing this model to be compatible with multiple headset designs. Simulations using inclusions of different sizes and positions were conducted on this model using perturbations of $\pm 10 \%$ and $\pm 1 \%$ to demonstrate the model's sensitivity to changes observed in stroke and functional activity, respectively. Finally, a software tool for delivering a working memory task with features for analysis of evoked responses and EIT/ECG data synchronization was developed for the human EIT-BI study. A protocol for imaging functional activity in humans was written and approved by Carleton's Research Ethics Board (REB). Unfortunately, due to the COVID-19 pandemic that occurred in the latter portion of this thesis, the human functional EIT-BI study could not be completed. The protocol, presented in appendix B, is planned to be executed once data collection can safely be resumed.

An automated electrode quality and data rejection tool (EQADR) was developed to facilitate clean and consistent measurement rejection for the stroke study, but was designed to be used with any dataset collected by SwissTom hardware. The goal of EQADR was to increase the accessibility of EIT to new researchers and to medical professionals, who may have minimal technical EIT experience but desire robust and reliable EIT images for downstream use.

Existing faulty electrode detection methods use contact impedance thresholds, though many low-quality measurements can be taken while contact impedance is within the normal range. The first major contribution of the automated electrode quality and data rejection tool (EQADR) was the robust, effective method used to detect faulty EIT electrodes and measurements. The pairing of EIT electrodes in measurements and stimulations made disentangling noisy from clean electrodes chal- 
lenging. A second major contribution was a method for determining which electrodes were truly noisy, and which electrodes were clean but appeared noisy by pairing with a noisy electrode. The second function of EQADR is an application of a compensation method for eliminating the effects of noisy electrodes or measurements on EIT images and was an implementation of existing work.

Lastly, appendix A presents a submitted article in which the effects of weighted restraint on the distribution of ventilation, and its putative role in arrest-related deaths, were tested. A shorter version of this article was published and presented in the $42^{\text {nd }}$ annual international conference of the IEEE Engineering in Medicine and Biology Society (2020). 


\section{Chapter 2}

\section{Literature Review}

\section{$2.1 \quad$ Introduction}

The human brain is an incredibly complex, fragile structure composed of billions of interconnected neurons and support cells, suspended within the skull by cerebrospinal fluid (CSF) [4]. Many brain imaging technologies have been developed to improve our basic understanding of its structure and function, and how these aspects are altered in disease. Emerging in this group is EIT, whose non-invasiveness, high temporal resolution, safety, low cost, and portability make it an attractive imaging modality. Unlike the ionizing radiation used to generate X-ray CT images, the high frequency, low amplitude alternating currents used in EIT are harmless to humans. EIT is therefore desirable in continuous or long-term monitoring scenarios, particularly for the brain. Its widespread clinical adoption has been limited by several physical and technical limitations of EIT, which are outlined in section 2.2.1. However, steady advances in hardware and software reveal EIT as a promising emergent medical imaging modality.

Brain EIT research has surged in the $21^{\text {st }}$ century due to improvements in EIT hardware, computing power, and sophistication of reconstruction algorithms [5]. A 
central challenge to imaging the brain with EIT is the ill-conditioning of the inverse image reconstruction problem, which is responsible for its high sensitivity to noise and low spatial resolution. Brain EIT research has thus far focussed on developing EIT for situations in which large-scale conductivity changes within the head occur, such as the detection of HT after ischemic strokes [2], detecting epileptic foci [6], imaging tumors [7], monitoring cerebral perfusion during surgery [8], and monitoring the level of consciousness during anaesthesia [9].

This chapter begins with an overview of EIT data collection and image reconstruction. The sources of conductivity changes in the head and brain and how these affect EIT image contrast, of which the movement of blood is a major contributor, are then discussed. The physiology of stroke will be introduced and the body of literature on EIT stroke imaging will be discussed.

The increasing interest in developing EIT for imaging changes in CBV can naturally be extended to measuring the smaller scale CBV changes of functional activity. An explanation of the hemodynamic response (HDR) and how it has been exploited to develop image contrast in existing functional imaging modalities will be discussed. The existing literature will be reviewed and the need for signal-to-noise ratio (SNR)enhancing algorithms and sophisticated models with realistic boundary shapes, tissue conductivity values, and tissue anisotropic properties for the forward solution to the conductivity distribution will be emphasized.

\subsection{Electrical Impedance Tomography}

An EIT image represents the internal distribution of electrical impedance (or inversely, conductivity) within a body of interest at a point in time. Given that biological tissues have distinct conductivity values, changes observed in the conductivity distribution 
over time reflect changes in how the tissues are distributed within the body. For example, a conductivity decrease is observed in the region of the lungs during inhalation, as they fill with non-conductive air. By applying controlled electrical currents between unique pairs of surface electrodes, while simultaneously measuring voltages from all other non-current-carrying electrodes, the impedance $Z$ encountered by a current $I$ along a path is related to the voltage measurements by $V=I Z$, where $Z$ has real-valued resistive component and complex-valued inductive and capacitive components.

It is worth mentioning here that the formulation above describes time-difference EIT (tdEIT), one of three EIT methods. Research from multi-frequency EIT (mfEIT) and frequency-difference EIT (fdEIT) methods will also be relevant to the discussion in this chapter. These two methods use the frequency-dependent conductivities of biological tissues to produce images, while time-difference EIT (tdEIT), as described above, images conductivity changes over time at a single frequency. MfEIT is an absolute imaging technique that does not use a reference frequency or frame, therefore mfEIT reconstructions are highly sensitive to modelling errors. The method of differencing from a reference frequency or frame in fdEIT and tdEIT respectively largely cancels out modelling errors, making these methods more robust than mfEIT. Unless the distinction between methods is explicitly needed, EIT will be used in the text to represent tdEIT.

EIT has applications in industrial and geophysical settings, but its development for medical imaging was first credited to Dr. Barber and Brown in the early 1980s [10]. Since then, EIT has been developed for a wide variety of medical imaging applications including: the monitoring of heart [11], lung [12], and brain [5] function, as well as imaging of tumours in breast [13] and brain [7] tissues. Additionally, the artificial introduction of $\mathrm{Na}^{+}$and $\mathrm{Cl}^{-}$ions as a saline bolus has been used as an EIT 
contrast agent. A saline solution is injected into the circulation and travels through the vasculature where it can be detected by EIT. This technique has been shown to accurately depict relative blood flow and perfusion both in the lung $[14,15,16]$ and in the brain [17].

\subsubsection{How EIT Works}

Tomographic images are generally formed by projecting interacting energy of known intensity from a source, through a system, from multiple unique angles, and recording the intensity of energy arriving at a detector. The internal composition of the system can then be approximated from the solution to an inverse problem, such as backprojection.

With hard-field imaging modalities such as X-ray CT, the assumption that the emitted energy is spatially uniform when arriving at the detector is valid. When interrogating a system with low-energy radiation such as electrical currents in EIT, this assumption breaks down. Current flowing through the system follows the path of least resistance, thus the current density decreases with distance from the stimulating electrodes. The consequence is a spatially non-uniform sensitivity that is high near the electrodes and low elsewhere, and ill-conditioning of the inverse problem.

Regularization techniques linearize the reconstruction problem and impart stability on images in the presence of conflicting measurements and instrumentation noise, seen in section 2.2.2. EIT image reconstruction begins with a model of the interrogated system that depicts current flow through that system with high fidelity. The a priori need for accurate structural information for regularization means EIT is predominately used for functional imaging.

Current flow through the body is impeded according to tissue-specific resistances in the plane of interest and can be represented by a continuous function. The fi- 
nite element method (FEM) of image reconstruction discretizes this function into triangular or tetrahedral elements, making the solution to the changing conductivity distribution computationally feasible. The sizing of elements in the finite element model (FEM) reflects the local electric field and current density. An example of an 8 electrode EIT setup on a circular body is shown in figure 2.1. The edges of each element are assigned a resistance value according to the electrical properties of the tissue in which that element is contained. The current density distribution created by a stimulating electrode pair can then be predicted by incorporating the path length and total resistance encountered over that path.

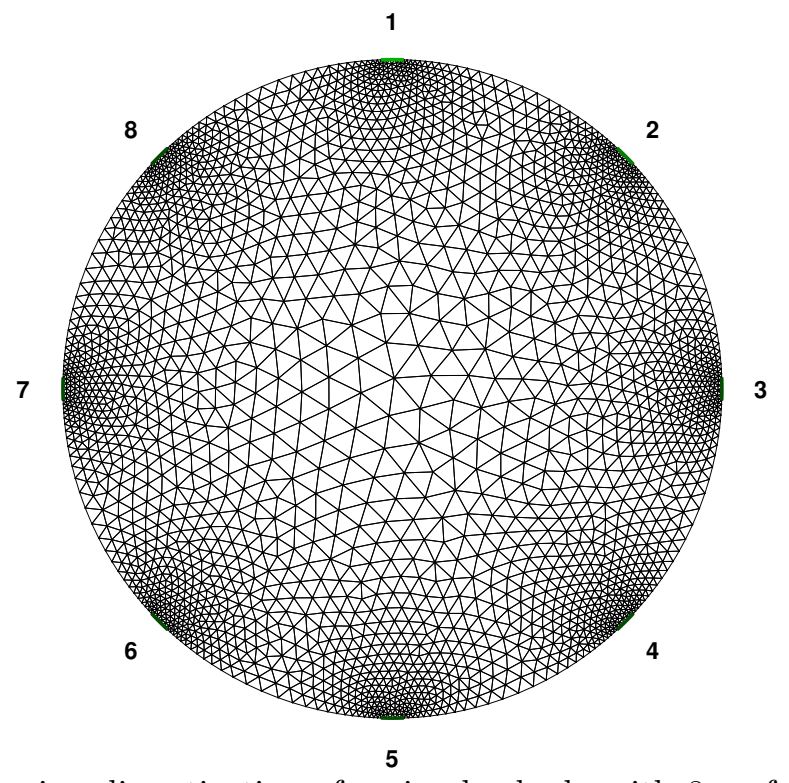

Figure 2.1: Model showing discretization of a circular body with 8 surface electrodes using the finite element method

EIT measurements proceed according to pre-determined stimulation (which pairs of electrodes carry current) and measurement (which pairs of electrodes measure potential) patterns. Stimulation and measurement patterns follow a "skip" setting that determines the number of skipped electrodes between each electrode pair. For example, a skip-1 pattern would indicate that electrodes $(1,3),(2,4),(3,5)$, etc. are pairs. The arrangement of the electrodes themselves on the body is up to the experimenter, 
permitting many possible electrode configurations.

Each EIT measurement uses two stimulating electrodes and two separate measuring electrodes, in a four-terminal configuration [18]. Impedance encountered at the surface-electrode interface creates an accumulation of charge when an electrode carries a current, resulting in electrode polarization and polarization potentials, which decrease over distance [19]. By using non current-carrying electrodes as measurement pairs, the contribution of polarization potentials to impedance-related voltage measurements is reduced, thereby increasing system sensitivity and accuracy.

A single EIT "projection" is formed by passing current between the first stimulating pair and recording voltages from each measurement pair, according to the measurement pattern. Current is then injected between the next stimulating pair, measurements are taken, and so forth for all stimulating pairs, until a single measurement frame has been collected.

An example of a measurement frame using a skip-1 stimulation and measurement pattern on a homogenous body is shown in the first two rows of figure 2.2. The equipotential lines from the currents injected by electrodes 1 and 3 are shown in the first two rows of figure 2.3. The third and fourth rows of figures 2.2 and 2.3 show the change in current flow and equipotential lines that occurred when a highly conductive circular inclusion was placed near electrode 3 , respectively.

\subsubsection{EIT Image Reconstruction}

The voltages measured at each measurement frame $d$ are related to the internal conductivity distribution $\sigma$ of the interrogated system by the equation:

$$
d=F \sigma+e
$$



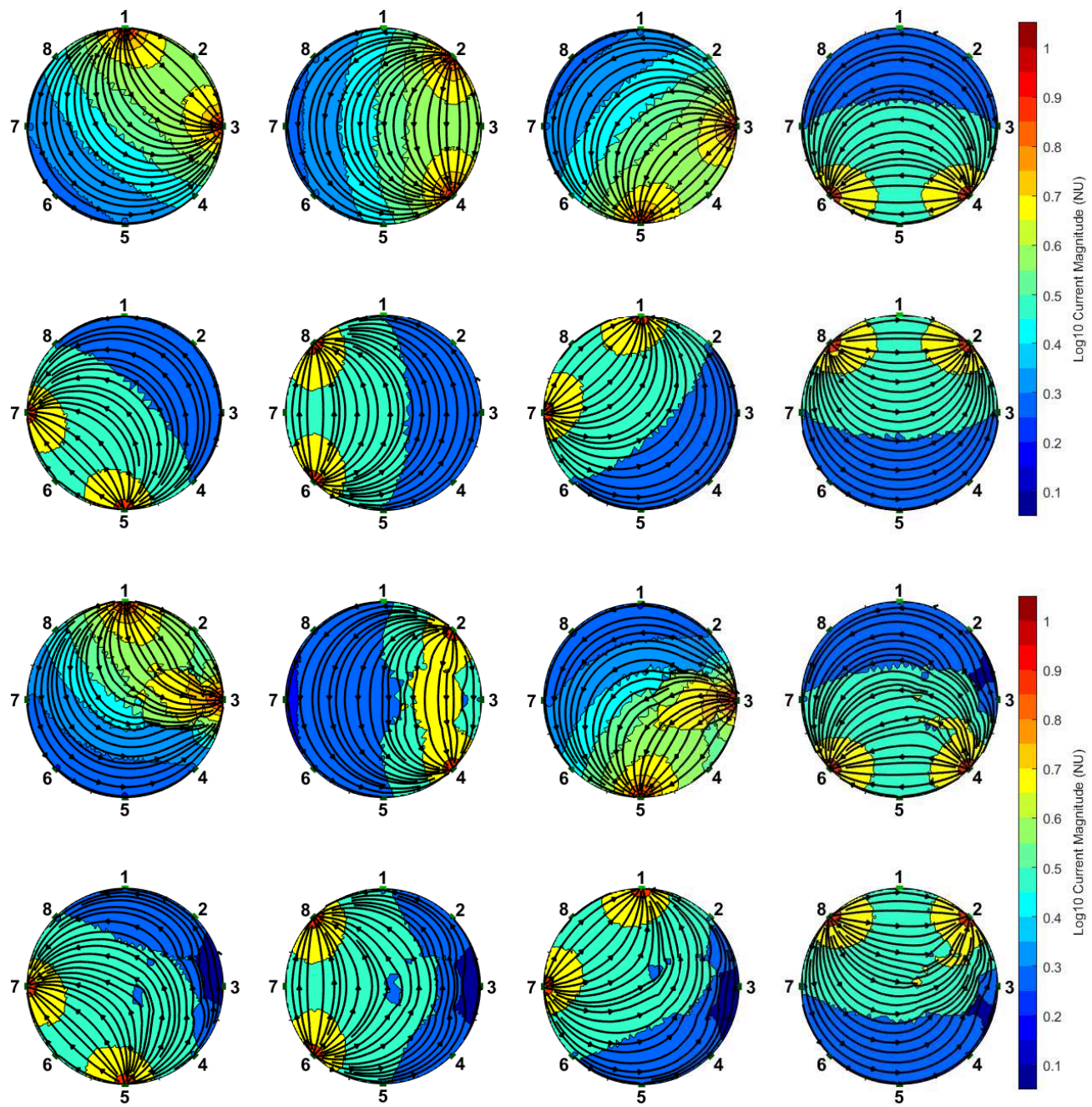

Figure 2.2: Simulated current lines (black) and current density (colored fill) for each stimulating pair in a skip-1 stimulation pattern. The model used here is the same as in figure 2.1. The first two rows show the simulated current when the model has a homogenous conductivity of 1 . The third and fourth rows show how current lines are altered when a highly conductive circular region is introduced near electrode 3 .

where $d$ is a vector of $m$ measurements, $F$ is the system's transfer function, $\sigma$ is a vector of length $n$, where $n$ is the number of pixels in the solution image, and $e$ is an error term representing random, uncorrelated instrumentation noise. The 

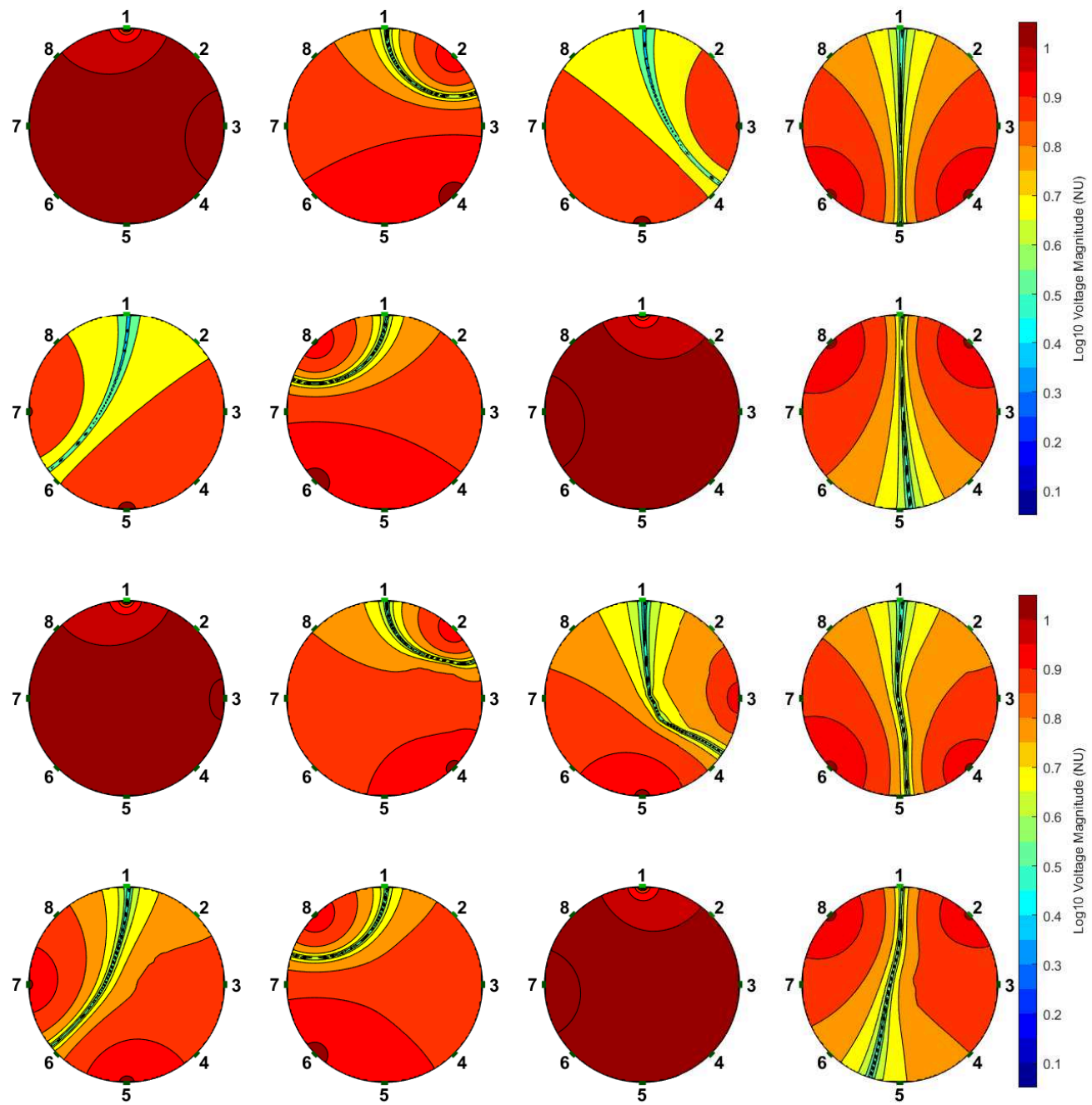

Figure 2.3: Simulated equipotential lines during stimulation between electrodes 1 and 3 for each measurement pair. A skip-1 measurement pattern was used, as shown by the stimulation pattern in figure 2.2. The model used here is the same as in figure 2.1. The first two rows show the simulated equipotential lines when the model has a homogenous conductivity of 1 . The third and fourth rows show how equipotential lines shift when a highly conductive circular region is introduced near electrode 3 .

image reconstruction problem is then linearized at a reference conductivity frame by determining the Jacobian matrix $J$, whose values represent the change from reference values $\Delta d$ of each measurement, for a given conductivity perturbation $\Delta \sigma$, at each 
image element $n$, and is size $m \times n$. Equation 2.1 can then be approximated by:

$$
\Delta d \approx J \Delta \sigma+e
$$

Determination of $J$ is the so-called forward problem, where changes in surface voltages are calculated from known conductivity changes, given a priori knowledge of the system, conveyed by the FEM, otherwise known as the forward model. The inverse problem, or "hard problem", is the reverse: determining the conductivity changes from changes in surface voltages. In majority of cases $n>m$. Such underdetermined systems have an infinite number of solutions because there are insufficient explanatory variables to account for the possible observable changes in the output image. Regularization methods must be used to redefine the problem from a system of linear equations as in equation 2.2 to the optimization of an objective function. Regularization of the linearized inverse problem can be expressed as a generalized Tikhonov regularization problem:

$$
\min _{\sigma}\left\{\|J \sigma-d\|_{2}^{2}+\lambda^{2}\|\mathcal{L} \sigma\|_{2}^{2}\right\}
$$

Here, the matrix $\mathcal{L}$ stabilizes image element values incorporating a priori knowledge that an element's sensitivity to changes in measurements is related to its distance from the body's center [20]. The regularization parameter $\lambda$ controls the weighting between the regularized solution norm $\|\mathcal{L} \sigma\|_{2}^{2}$ and the residual norm $\|J \sigma-d\|_{2}^{2}$. Minimizing the residual norm finds the solution vector $\sigma$ that is the best fit of the data $d$. This is not always the best solution because the measurements contain some level of noise $e$, and the Riemann-Lebesgue lemma shows that when solving for the inverse, spectral components of the input signal are amplified in the output signal, but amplification 
occurs non-uniformly in a way that is directly related to frequency [21]. This means that high frequency components of the input signal, such as instrumentation noise, are amplified more than lower frequencies in the solution. This poses a problem because these lower frequencies are typically the signals of interest in biological systems. The hyperparameter $\lambda$ allows weight to be placed on minimization of the solution norm, which effectively dampens the amplification of these high frequency components. The result is a more stable solution that is less sensitive to instrumentation noise and follows well our a priori knowledge of the biological systems under examination. The reconstructed image from the simulated data in figures 2.2 and 2.3 is shown in figure 2.4 .
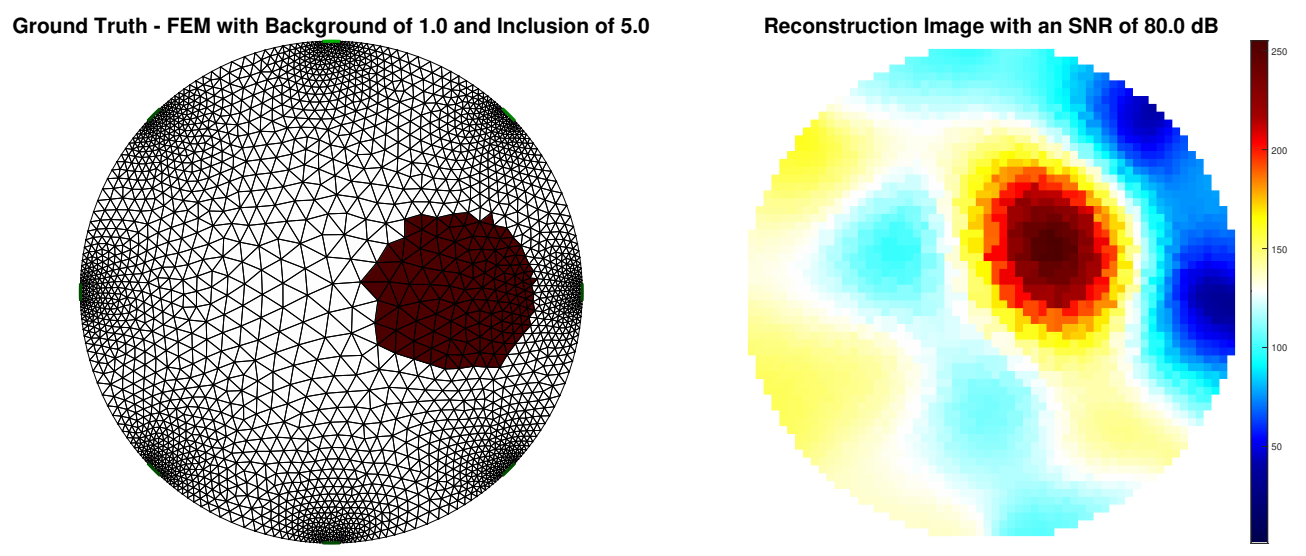

Figure 2.4: Reconstructed image from the voltages shown in figure 2.3 showing the highly conductive inclusion on the right side of the circle, using a SNR of $80 \mathrm{~dB}$. The inclusion can be seen on the right side in the reconstructed image. On the right side of the inclusion, a ringing artifact can be seen because the area beside the inclusion appears relatively less conductive than the inclusion itself to far away electrodes (figure 2.2, last 5 images). 


\subsection{Sources of EIT Brain Conductivity Changes}

Conductivity changes within the head result from changes in tissue distribution, or from ion movement across cell membranes during neuronal depolarization. Conductivity changes in the latter case are small and currently must be measured from cortical surface electrodes $[22,23]$. Such studies have demonstrated how the high temporal resolution afforded by EIT enables measurement of fast electrical activity within the brain. However, in the interest of developing non-invasive applications of EIT, the former source of contrast, which exists on a scale that can be measured from outside of the skull instead of directly on the cortical surface, will be the focus of the discussion here. The movement of fluid tissues such as blood and CSF through vessels and compartments within the head, and their high conductivities relative to other tissues (shown in table 2.1) are the major contributors to this signal. The bulk movement of ions and water in cerebral edema and the artificial injection of ions into the circulation with EIT saline contrast agents are other sources of EIT contrast.

\begin{tabular}{ll} 
Tissue & Conducivity $\left(S \cdot \mathrm{m}^{-1}\right)$ \\
\hline Scalp & 0.41 \\
\hline White matter & 0.22 \\
\hline Grey matter & 0.47 \\
\hline CSF & 1.71 \\
\hline Blood & 0.57 \\
\hline Skull & $1.6 \times 10^{-2}$ \\
\hline Air & $1.0 \times 10^{-5}$
\end{tabular}

Table 2.1: Conductivity values for tissues of the head, obtained from the column of weighted means in table 3 of a meta-analysis on measured conductivity values for human head tissues [24].

\subsubsection{Cerebral Edema}

Cerebral edema is a state of homeostatic imbalance caused by failure to maintain normal osmotic gradients, leading to an influx of water into the intracellular space. 
In stroke, infarction may lead to cytotoxic cerebral edema, where ATP-dependent $\mathrm{Na}^{+} / \mathrm{K}^{+}$antiporter membrane pumps fail due to cell death and lead to cellular swelling [25]. In these conditions, the extracellular space has been shown to shrink by as much as $50 \%$ [26]. Cerebral edema after ischemia has been reported to create conductivity decreases of $9.7 \pm 2.5 \%$ when measured from the scalp and decreases of $110.7 \pm 20.1 \%$ when measured on the cortex in cases of mild hypoxia [27]. These decreases can become $33 \pm 9.4 \%$ and $142 \pm 50.2 \%$ in cases of severe hypoxia when measured from the scalp and cortex respectively [27]. Cerebral edema may also occur in epilepsy when widespread synchronized neuronal firing leads to a massive intracellular influx of $\mathrm{Na}^{+}$, driving water in the same direction [28].

The brain's surrounding by the highly resistive skull provides a barrier to EIT imaging; most applied currents do not penetrate the skull, but instead are shunted by the scalp. Moreover, currents penetrating the skull may be shunted by the CSF, flowing around rather than through the white and grey matter [29]. Several studies have investigated the percentage of applied current that enters the parenchyma; this was shown to be on average $15 \%$ of applied current when tested in anesthetized rabbits [30] and $36 \%$ in live pigs [31]. In humans, a tank study performed measurements on a human head phantom both with and without human skull [32] and showed that impedance changes within the brain were attenuated by a factor of $8.2,5.5$, and 5.6 for anterior, central, posterior coronal compartments $(12.2 \%, 18.2 \%$, and $17.9 \%$ penetration) respectively. In short, the skull is the reason why EIT-BI is so difficult.

\subsection{Stroke Imaging}

The pathological state of reduced blood supply to the brain is known as stroke and occurs by two mechanisms: blockage of an artery (ischemia) or rupturing of a blood 
vessel causing internal bleeding (hemorrhage). Stroke is the second largest cause of death worldwide and causes morbidity in up to $50 \%$ of survivors [1].

Acute ischemic strokes are more common than hemorrhagic, and comprise approximately $80 \%$ of strokes [33]. They are treated with plasminogen-activating drugs that break down clots and allow the return of blood flow to the affected areas, or by mechanical thrombectomy. Hemorrhagic strokes are treated by taking measures to stop the bleeding, controlling blood pressure, and limiting the damage caused by the hematoma to the surrounding tissues [34]. Diagnostic imaging is critical for ruling out hemorrhage in patients presenting with stroke symptoms, considering that thrombolytic drugs counteract the body's attempts to control hemorrhage through clot formation, and could worsen outcomes in these persons. Rapid diagnosis is critical for ischemic stroke treatment because plasminogen-activating factor must be administered within 6 hours of symptom onset [3]. After this early hyperacute phase, treatment poses an increased risk of HT [35]. Minimizing the time to treatment is also an important factor for decreasing the likelihood of cognitive and functional deficits in survivors of ischemic stroke [35].

Diagnosis and classification of stroke type is normally carried out by a combination of perfusion $\mathrm{CT}$ and $\mathrm{CT}$ angiography [2]. In cases where the time of stroke occurrence is unknown (such as during sleep, or if symptoms occurred late after onset), MRI is used to determine the age of the stroke, which informs the appropriate treatment decision [3].

Stroke also occurs perioperatively in anesthetized patients. While the overall incidence of perioperative stroke is only $0.7 \%$, it can be as high as $10 \%$ in cardiac and vascular surgery. Intraoperative stroke is a devastating complication that leads to a 6-fold increase in mortality [36]. During anesthesia, diagnosis of stroke is especially difficult since the effects of anesthetics prevent common stroke symptoms like pares- 
thesia, confusion, or speech difficulties from being observed. A continuous monitoring device of cerebral perfusion for stroke in perioperative patients would therefore be of tremendous interest. The suitability of EIT as a tool for stroke detection and classification has been a subject of ongoing research. If successful, EIT could be used for continuous monitoring of stroke patients or during anaesthesia, alerting medical staff if HT or intraoperative stroke is detected.

\subsubsection{Stroke Imaging with EIT}

Early proof-of-principle studies confirmed that changes in rCBV could be imaged with EIT. Holder showed that cerebral ischemia in a rat cortex resulted in conductivity decreases of 15-60\% when measured from cortical electrodes [37]. These amplitudes were 5-10 times less when measured from scalp electrodes, but still within the limit of detectability [37].

Strokes have been challenging to image with tdEIT because a healthy reference image is not available for reference when a patient presents with symptoms [38]. FdEIT studies have revealed that healthy and ischemic spectral profiles differ most at $100 \mathrm{~Hz}$ and below [39, 40, 41]. Unfortunately these frequencies at normal amplitudes are perceptible to humans, and reducing power at these frequencies comes with the cost of reduced image quality [40]. This is a key disadvantage of fdEIT compared to tdEIT. Imaging of hemorrhagic stroke has been largely successful with fdEIT and tdEIT methods, however imaging ischemia has proven challenging. Due to the high prevalence of ischemia among strokes and the desire to keep EIT imaging comfortable and reliable, methods for imaging ischemia with tdEIT were developed and tested in the current work. 


\section{Imaging Hemorrhagic Stroke with EIT}

When hemorrhage occurs in the arachnoid space or in the cerebral ventricles, the highly conductive CSF is displaced by less conductive blood, and conductivity observed by EIT decreases. Subarachnoid hematoma has been successfully imaged from scalp electrodes in piglets injected with blood in the subarachnoid space with tdEIT [42]. The same group was able to repeatedly image conductivity changes due to irrigation of hematoma with $5 \%$ dextrose in water, injected in the cranium, with tdEIT during surgery [43]. Other studies have detected intraventricular hemorrhage using tdEIT, with high specificity, from surface electrodes [44]. Parenchymal hemorrhage, simulated by injections of unclotted arterial blood into the white matter of piglets, has also been detected as a conductivity increase in $6 / 8$ piglets in the correct location using tdEIT and a central internal electrode [45]. FdEIT has successfully imaged hemorrhage. In a study by Dowrick et al., conductivity changes from hemorrhages ranging from $2-52 \mu \mathrm{L}$, induced in rats, were measured at the correct magnitude for volumes $>4 \mu \mathrm{L}$, and localized correctly for volumes $>6 \mu \mathrm{L}[33]$.

\section{Imaging Ischemic Stroke with EIT}

In spite of the successes of imaging hemorrhage, imaging ischemia with EIT has been more challenging. FdEIT studies have shown differences in impedance spectra between healthy and ischemic brain tissue [40], with the largest differences in the sub-100 Hz range $[39,40]$. Attempts at imaging ischemia with fdEIT have yet to be successful [33]. In the cited study, the direction of conductivity change observed in simulated ischemia was incorrect in all cases. In spite of the localization of ischemia being correct in 6/7 cases, too many image artifacts were observed to claim this as a success [33]. In another study, global conductivity decreases were observed in 
the brains of $8 / 8$ piglets when cerebral blood flow was arrested by euthanasia [45], however complete circulatory arrest has limited applicability to non-lethal ischemia in the target patient population.

This survey of the literature has shown that although EIT has imaged cerebral hemorrhage, little success has been achieved with imaging ischemia. Considering that ischemia occurs in approximately $80 \%$ of all acute stroke cases [33], methods for improving ischemia imaging with EIT have the potential to offer a large benefit to the treatment and management of this disease.

\subsection{Functional Neuroimaging}

The goals of functional neuroimaging are to understand how the brain receives, processes, and responds to changing internal and external stimuli. The applications of functional neuroimaging technologies include but are not limited to: fundamental research on emotional and cognitive states, consciousness, sleep, brain development, localizing and predicting occurrence of epileptic seizures, assessing the impacts of brain injury, and brain-computer interfacing.

\subsubsection{The Hemodynamic Response}

The HDR is the process by which localized transient increases in CBV and cerebral blood flow $(\mathrm{CBF})$ occur due to increased neural activity.

At its most fundamental level, the brain is made up of interconnected neurons that communicate at synapses via the release of neurotransmitters triggered by action potentials. The concerted action potentials between the billions of neurons in the human brain ultimately results in cognition, sensations, motor control, and consciousness. Neurons at rest are not quiescent, but interact via spontaneously-generated 
action potentials [46], fuelled by the hydrolysis of adenosine-triphosphate (ATP) to adenosine-diphosphate (ADP). Cellular ATP stores are primarily replenished by the oxidative phosphorylation of ADP to ATP in the mitochondria, a process fuelled by the oxidation of glucose [4]. The $\mathrm{O}_{2}$ for this pathway in turn is obtained from the deoxygenation of hemoglobin $(\mathrm{Hb})$ to deoxy-hemoglobin $(\mathrm{dHb})$ in the blood.

A group of neurons recruited for a task experience a transient increased rate of signalling, and thus, an increased rate of ATP and $\mathrm{O}_{2}$ consumption. This causes a localized decrease and increase in $[\mathrm{Hb}]$ and $[\mathrm{dHb}]$ respectively, in the nearby capillary bed [47]. The accumulated waste products from cellular respiration $\left(\mathrm{CO}_{2}, \mathrm{NO}\right.$, and $\mathrm{H}_{2}$ ) act as chemical signals, triggering vasodilation of arteries feeding the capillary bed of the recruited neurons. Blood flow to the area increases along with [Hb] [47].

Many functional imaging modalities such as fMRI, PET and SPECT exploit at least one aspect of the HDR to produce image contrast, however others such as EEG and MEG measure the electrical and magnetic signals of action potentials, respectively.

\subsubsection{Principles of Brain Imaging and Major Modalities}

\section{Functional Magnetic Resonance Imaging (fMRI)}

FMRI uses the difference in magnetic properties of $\mathrm{Hb}$ and $\mathrm{dHb}$ to achieve image contrast. $\mathrm{Hb}$ molecules contain 4 heme groups, each with an oxygen-binding iron atom. Heme groups are weakly attracted to magnetic fields when bound to oxygen, and are nearly magnetically indistinguishable from normal brain tissue [47]. Conversely, deoxygenated heme groups are weakly repelled by magnetic fields and contrast with

normal brain tissues. The ratio of $[\mathrm{Hb}] /[\mathrm{dHb}]$ is the so-called blood-oxygen level dependent (BOLD) signal and is the source of image contrast in fMRI. Changes in 
local blood volume and cellular metabolism both contribute to the $[\mathrm{Hb}] /[\mathrm{dHb}]$ and therefore the BOLD signal [48]. FMRI has a spatial resolution of $1 \mathrm{~mm}$, a temporal resolution of $1 \mathrm{~s}$, and can image the full depth of the brain [49].

\section{8-Fluoro-Deoxy-Glucose Positron-Emission Tomography $\left({ }^{18}\right.$ FDG-PET $)$}

${ }^{18}$ FDG-PET uses the cellular uptake of radio-labelled glucose molecules, such as ${ }^{18}$ fluorodeoxyglucose $\left({ }^{18} \mathrm{FDG}\right)$, to image functional activity. ${ }^{18} \mathrm{FDG}$ behaves like normal glucose in humans, but emits positrons that annihilate into two $\gamma$ photons, emitted at $180^{\circ}$ from one another, after colliding into an electron. The coincident detection of two $\gamma$ photons, at the correct energy level, on opposite sides of the detector, registers as a true emission event. The position of the positron-electron collision is calculated from the delay between the detection of the first and second $\gamma$ photons. The reconstructed images represent cellular glucose uptake and relative CBF in the area of interest $[48,49]$. PET has a spatial resolution similar to that of fMRI, a temporal resolution of $10^{2} \mathrm{~s}$, and can image the full depth of the brain [49].

\section{Single-Photon Emission Computed Tomography (SPECT)}

SPECT images functional activity by tracing the relative uptake of $\gamma$-emitting radionuclides by neurons. When injected, the radionuclides, such as ${ }^{99 m}$ Tc-hexamethyl propylene amine oxime (HMPAO), are lipophilic and able to cross the blood-brain barrier (BBB). They are then metabolized into lipophobic compounds that remain within the BBB, circulating in the vasculature until being cleared cells [50]. This technique allows for perfusion imaging within the cerebral cortex during the 90-120 s period in which radionuclide uptake occurs [50]. The temporal and spatial resolution of SPECT is similar to those of PET, however the need for coincident detection in SPECT results in lower image quality than PET. 


\section{Electroencephalogram (EEG)}

EEG measures changes in voltages from aggregated action potentials in the brain, from surface electrodes. EEG has been a powerful tool for understanding aspects of brain function but has a limited ability measure spontaneous activity of deeper brain structures [51]. It has a $10^{-3} \mathrm{~s}$ temporal resolution and a $10^{2} \mathrm{~mm}$ spatial resolution.

\subsubsection{Functional Neuroimaging with EIT}

EIT has the potential to measure functional activity within the brain from the CBV changes that occur as part of the HDR. The difference in conductivity of blood and other tissues in the head makes this possible (see table 2.1). An activated brain region will see a localized increase in CBV in the surrounding capillary bed, and a corresponding conductivity increase. These changes, however, are small and at the limit of detection of current EIT systems [5].

To investigate the validity of measuring functional activity with EIT, a preliminary study measured brain conductivity changes from evoked responses in 8 rabbits from electrodes on the exposed cortex, thereby eliminated the resistive barrier imposed by the skull. A consistent conductivity increase of $\sim 3 \%$ was observed in the correct brain location in $8 / 8$ rabbits that were either subjected to a visual or somatosensory stimulus. These conductivity increases were in agreement with the theoretical expectation that blood moving into a tissue of lower conductivity would result in an overall conductivity increase [52].

This study was followed by a 2D EIT experiment of brain function in humans, using a similar paradigm of somatosensory and visual stimuli [53]. The SNR of electrode measurement pairs for each 6 min experiment were improved by averaging together all 96 measurement frames collected during that experiment, during either a $3 \mathrm{~Hz}$ 
electrical stimulation of the wrist's median nerve, or an $8 \mathrm{~Hz}$ oscillating checkerboard visual stimulus. Six experiments per stimulus were recorded, and significant conductivity changes were defined as an electrode combination having 5 or more averaged experiments that were significantly different than baseline. Reproducible impedance changes were observed in all experiments, and in $20 \%$ of measurements, significant changes were observed. Of the experiments with significant conductivity changes, $50 \%$ were localized to the correct area.

Shortly after, Tidswell et al. collected EIT data from humans using a 3D imaging configuration under a protocol similar to that in [53] and reconstructed on a homogeneous sphere. Again, significant conductivity changes in the correct location were observed for $5 / 9$ visual $(\mathrm{n}=13), 4 / 8$ motor $(\mathrm{n}=20)$, and $1 / 2$ somatosensory $(\mathrm{n}=18)$ stimuli [32], but were not consistent with the conductivity increases expected to occur during functional activity. These errors were attributed to the use of a homogenous, isotropic sphere reconstruction model rather than a realistic model depicting the concentric layers of the scalp, skull, and brain with their respective conductivity values.

Tidswell et al. later isolated the effect of the skull on reconstructions though a tank study that compared the localization accuracy of a conductive sponge using a headshaped phantom either with, or without a skull, when the forward model assumed that the head was a homogeneous isotropic sphere [54]. The presence of the skull increased localization errors by $7-8 \%$ with a tendency for foci to shift towards the center of the images. Bagshaw et al. applied these results by reconstructing the data from [32] using a forward model of concentric spheres, that incorporated the scalp, skull, brain, and their conductivity values. The images produced by the shelled spherical model were compared those produced by a homogeneous sphere, showing that the shelled sphere model improved reconstructions, and showed consistent conductivity increases in the correct location for the motor and visual tasks, even in instances where no 
significant change had been previously observed with the homogenous sphere model $[55]$.

More recently, EIT has been shown to detect hemispherical changes in CBV and indicators of brain damage in humans, from surface electrodes, during surgery [8]. EIT has also shown potential for measuring other changes in large-scale cognitive states, such as loss of consciousness in horses [56] and in humans [9] when under anaesthesia. These results are promising and show the utility of EIT as a brain imaging tool.

In summary, the principle of measuring functional activity with EIT using the HDR has been demonstrated using electrodes on the surface of the brain. Results in human studies with electrodes placed on the scalp have shown the importance of using realistic head models and use SNR-improving averaging techniques to increase overall sensitivity and image quality.

\subsection{Advanced EIT Electrode Arrays for Brain Imaging}

With EIT-BI comes the unique challenge of achieving acceptable electrode contact with the scalp and low contact impedances $\left(C_{Z}\right)$ in the presence of thick hair. Standard $\mathrm{Ag} / \mathrm{AgCl}$ electrocardiogram (ECG)-type electrodes used in EIT are not suitable for use over hair because they lay on top rather than penetrating through and between hairs. Studies using ECG-type electrodes have had to shave participants' heads to acquire good signal quality [8], and are impractical. Therefore, the development of an electrode array that achieves adequate scalp contact in the presence of thick hair would greatly increase the accessibility of EIT-BI.

An EIT electrode array must be quick to set up, comfortable, have low $C_{Z}$ between 
the electrodes and scalp, be robust to motion artifacts and noise, and must be easily cleaned and/or sterilized between uses.

Low $C_{Z}$ is critical because EIT electrodes are used for both stimulation and measurement [57], and the lower the $C_{Z}$ at the electrode-skin interface, the more current will flow through the body, and the lower the polarization potentials will be. Studies have evaluated the performance of $\mathrm{Ag} / \mathrm{AgCl}$ EEG electrodes in EIT setups, finding that powder $\mathrm{Ag} / \mathrm{AgCl}$ electrodes perform the best $[58,19]$. Another study tested four different electrode arrays, each with a different electrode type, $(\mathrm{Ag} / \mathrm{AgCl}$ electrode gel with cup electrodes, saline-soaked sponge, headcap with wells that took a special electrode gel, and a conventional $\mathrm{Ag} / \mathrm{AgCl}$ electrode cup and gel setup) and showed that hydrogel electrodes produced the cleanest signal with low contact impedance [57]. In all cases skin abrasion was demonstrated to be an important step for lowering electrode contact impedance.

A servo-controlled helmet with electrode contacts made of 316 stainless steel, $10 \mathrm{~mm}$ in diameter was recently developed by Avery [28]. This helmet reduces $C_{Z}$ by abrading the scalp and through action of servos that also serve to maintain constant pressure of the electrodes against the scalp. Avery found that the greatest reduction in $C_{Z}$ was achieved after one rotation of each electrode against the scalp. Subsequent rotations saw little to no reductions in $C_{Z}$. Pyramidal and smooth electrode contacts had similar $C_{Z}$ reductions after the first rotation, and level of electrode compression affected decrease in $C_{Z}$. Therefore, rotation and compression of electrode against head can reduce $C_{Z}$ considerably, even with smooth electrode head, though rough head reduces $C_{Z}$ slightly more with additional rotations. Overall this headset was found to be too complex and heavy, but demonstrated a good design that allowed rotation of electrode contacts to abrade the skin, fixed in a helmet that reduces setup time and gives consistent electrode location and spacing on different subjects. Further 
research in the design and testing of electrode headset arrays will not only make brain EIT research easier to conduct but will help with clinical adoption once the technology has matured in this application. 


\section{Chapter 3}

\section{Perfusion-Based Methods for}

\section{Detecting Cerebral Ischemia with}

\section{Electrical Impedance Tomography}

This chapter presents an article in final preparation, in which two novel perfusionbased methods for detecting cerebral ischemia with EIT were developed and tested. The experiments were performed by the team at UKE Hamburg in a feasibility study for a novel high flow cold air-cooling protocol of the porcine brain using MRI temperature mapping. The EIT data was collected in parallel to the data used in the feasibility study, and the present article represents the first analysis and presentation of the EIT data. The algorithm design, image tissue segmentation, creation of the reconstruction models, EIT data processing, image reconstruction, and analysis were performed by the author in the current work. The ethics, study design, animal care sections, and the arterial blood pressure paragraph in the data collection section of the manuscript were written by the UKE team. The remainder of the manuscript was written by the author. 
Title:

Perfusion-Based Methods for Detecting Cerebral Ischemia with Electrical Impedance Tomography

Authors:

Mark Campbell, Christoph Behem, Constantin Trepte, Andy Adler Acknowledgements:

The authors would like to thank Dr. med. vet. Aline Reitmeier and Mrs. Jutta Dammann for providing pre- and perioperative animal care and their technical assistance on animal handling.

Funding:

The study was performed in parallel with a feasibility study for a novel high flow cold air cooling protocol of the porcine brain using MRI temperature mapping, which was funded by the Federal Ministry of Education and Research (BMBF) Germany (grant number 031A530). The Fabius MRI anaesthesia machine was provided free of charge by Dräger Medical GmbH (Luebeck, Germany). The EIT-belt and adapting hard- and software for EIT measurements were provided free of charge by Swisstom AG (Landquart, Switzerland). Additional costs were supported by the Department of Anesthesiology, University Medical Centre Hamburg-Eppendorf.

\subsection{Introduction}

The pathological state of reduced blood supply to the brain is known as stroke and occurs by two mechanisms: blockage of an artery (ischemia) or rupturing of a blood vessel causing internal bleeding (hemorrhage). Stroke is the second leading cause of death worldwide and causes morbidities in up to $50 \%$ of survivors [1]. Acute ischemic 
strokes are more common than hemorrhagic, and comprise approximately $80 \%$ of strokes [33]. Rapid diagnosis is critical for ischemic stroke treatment because plasminogen activating factor must be administered no later than 6 hours after symptom onset [3]. After this hyperacute phase, treatment poses an increased risk of hemorrhagic transformation [35]. CT and MR imaging are the current standards for diagnosis and classification of stroke, but these imaging modalities are not practical for long-term monitoring of hemorrhagic transformation in susceptible patients due to their ionizing radiation and cost, respectively. Moreover, stroke also occurs perioperatively in anesthetized patients. While the overall incidence of perioperative stroke is only $0.7 \%$, it can be as high as $10 \%$ in patients undergoing cardiac or vascular surgery. Intraoperative stroke is a devastating complication that leads to a 6-fold increase in mortality [36]. During anesthesia, diagnosis of stroke is especially difficult since the effects of anesthetics prevent common stroke symptoms like paresthesia, confusion, or speech difficulties from being observed. A device for the continuous monitoring of cerebral perfusion for stroke and perioperative patients would therefore be of tremendous interest.

Electrical impedance tomography (EIT) is a capable of imaging the changing internal conductivity distribution of a body by probing with small insensible currents passed between changing pairs of electrodes, while measuring resulting surface voltages from other non-injecting electrodes. EIT systems are well-suited for continuous long-term monitoring applications due to their safety, high temporal resolution, small size, and relative low cost. EIT has been shown to successfully detect simulated hemorrhagic stroke by exploiting the displacement of highly conductive CSF with lower conductivity blood in a piglet model [42, 43, 44]. Ischemic stroke has been challenging to image with EIT because a healthy reference image is not available for comparison with images taken when a patient presents with symptoms [33, 45]. Multi-frequency 
and frequency difference EIT have shown potential for imaging hemorrhage and ischemia $[40,33,38,39,41]$, however healthy and ischemic spectral profiles differ most at $100 \mathrm{~Hz}$ and below. These frequencies at normal amplitudes are perceptible to humans, and reducing power comes with the cost of reduced image quality.

To overcome the difficulty of imaging ischemia, the goal of this work was to develop and test two new perfusion-based time-difference EIT-BI approaches in a pig model. The first approach used arterial blood pressure (ABP)-synchronized EIT data to align multiple EIT sequences at the ABP peak to produce a series of images showing the average perfusion-related conductivity change within the brain with improved signal to noise ratio (SNR). The second approach used a hypertonic saline bolus injected in the right atrium, acting as an EIT contrast agent to image cerebral of blood flow.

The use of an ABP signal to decrease signal variability by averaging images from systolic and diastolic phases of the heart has been previously explored [45]. Our approach differs by first defining a cardiac cycle as a triad of diastole-systole-diastole, then re-referencing images from each cardiac cycle under observation to the mean of its diastoles, achieving sequences that are independent of the original reference used for image reconstruction. These sequences are then aligned at systole and ensembleaveraged to improve SNR. The result is a sequence of images showing the average dynamics of conductivity over a cardiac cycle. This sequence was then compared to DW-MR images to assess their reflection of CBV. The use of a hypertonic saline bolus as an EIT contrast agent has been shown to accurately depict relative blood flow and perfusion in the lungs $[14,15,16]$ and reveal changes in cerebral perfusion from cortical electrodes [17]. Here, we present the results from saline bolus used as EIT contrast for monitoring cerebral perfusion from surface electrodes for the first time. 


\subsection{Methods}

Two EIT perfusion-based methods for imaging ischemia with time-difference EIT were developed and tested, using data collected from 5 anesthetized adult pigs [59] (animals 1-5) before, immediately after, and 30-360 minutes after induction of cerebral perfusion deficits (figure 3.1 panel A). Each EIT measurement consisted of four experimental phases in which EIT and ABP data were collected continuously, and are shown in figure 3.1 panel B. Finally, the EIT belt was then removed and MRI (Siemens, Skyra) was used to quantify perfusion deficits and infarcts, which were then compared to the EIT images from each the two perfusion-based imaging approaches described in this section.

\subsubsection{Ethics}

The study was approved by the Governmental Commission on the Care and Use of Animals of the City of Hamburg (Reference-No. 15/016). The animals received care in compliance with the 'Guide for the Care and Use of Laboratory Animals' (NIH publication No. 86-23, revised 2011) as well as FELASA guidelines and recommendations and experiments were carried out according to the ARRIVE guidelines $[60,61]$.

\subsubsection{Study Design}

The study was conducted as an observational pilot study in 5 anaesthetized female domestic pigs (German landrace) weighing $44.5 \pm 1.8 \mathrm{~kg}$. This study was performed in combination with a feasibility study for a novel high flow cold air-cooling protocol of the porcine brain using MRI temperature mapping in accordance to ARRIVE and FELASA guidelines and 3Rs-principles reducing animal number. Results of the high 
flow cold air cooling protocol have been previously reported [59].

\subsubsection{Animal Care, Anaesthesia and Surgical Procedures}

The animals were purchased at Viehvermarktung Horst eG (Horst/Holst, Germany). Animals were brought to the animal care facilities at least 7 days prior to experiments and were housed in accordance to animal welfare recommendations. Animals were given food and water ad libitum and health status was regularly assessed by the responsible veterinarian. A fasting time of 12 hours was maintained prior to the experiments. For anesthesia induction all animals received intramuscular injections of ketamine $10 \mathrm{mg} \mathrm{kg}^{-1}$ bodyweight and xylazine $4 \mathrm{mg} \mathrm{kg}^{-1}$ bodyweight ${ }^{-1}$ for premedication. Thereafter, all animals were surgically tracheotomized and intubated via the tracheotomy ostium.

Anaesthesia was maintained by continuous infusion of propofol (8 $\mathrm{mg} \mathrm{kg}^{-1}$ bodyweight $^{-1}$ hour $\left.^{-1}\right)$ and fentanyl $\left(50 \mathrm{\mu g} \mathrm{kg}^{-1}\right.$ bodyweight $^{-1}$ hour $\left.^{-1}\right)$. Adequacy of anaesthesia was assessed by careful observation of vital signs, ventilation parameters, and by absence of any movements during the protocol with special attention to phases of surgical stimulus. Additional bolus doses of fentanyl $(50 \mathrm{\mu g})$ were given if there was any indication of pain or distress. Pancuronium (0.1 $\mathrm{mg} \mathrm{kg}^{-1}$ bodyweight $\left.^{-1}\right)$ was only given for tracheotomy.

A volume-controlled ventilation was established using a fraction of inspired oxygen (FiO2) of 0.5 , tidal volumes of $8 \mathrm{ml} \mathrm{kg}^{-1}$ bodyweight $^{-1}$ and a positive end-expiratory pressure of $8 \mathrm{cmH}_{2} \mathrm{O}$ and ventilator frequency was adjusted to maintain an arterial carbon dioxide partial pressure (paCO2) of 35-45 mmHg (Fabius MRIR), Dräger Medical, Lübeck, Germany). All animals were placed in supine position on a warming blanket to prevent heat loss.

Two central venous catheters were introduced into the right external jugular vein 
under ultrasound guidance, one for volume administration (8 Fr introducer sheath) and the other for maintenance of anaesthesia and catecholamine administration ( $7 \mathrm{Fr}$ 3-lumen central venous catheter). An arterial line was placed in either the right or left femoral artery (22 G Arterial Leader Cath). For monitoring of heart rate and oxygen saturation a 5-lead electrocardiogram and pulse oximeter were connected to the adapted hardware (Infinity Deltaß, Dräger Medical, Lübeck, Germany). For monitoring and recording of arterial pressure, the invasive pressure catheter was connected via a fluid-filled arterial pressure line and attached to a micro-tip catheter (Millar Micro-Tip $\AA$ pressure catheters, Houston, Texas, USA). The micro-tip catheter was zeroed to ambient pressure, levelled at the right atrium, and calibrated via 2-point calibration technique prior to measurements.

\subsubsection{Data Collection}

Arterial blood pressure (ABP) data was recorded by adapted hardware from ADInstruments (ADInstrumentsPowerLabß, ADInstruments Ltd., Oxford, UK). Data analysis was performed off-line using LabChart $\AA$ ) software (LabChart Pro, version 8, ADInstruments Ltd., Oxford, UK). Norepinephrine was administered to maintain mean arterial pressure $>65 \mathrm{mmHg}$. Stroke was induced using an experimental precipitating hydrophobic injectable liquid $\left(\mathrm{PHIL}^{\mathrm{TM}}\right.$, MicroVention Terumo, Tustin, $\mathrm{CA})$. The entire experimental procedure and handling of the animals was supervised by the responsible veterinarian. After completion of the study protocol all animals were sacrificed during deep anaesthesia by fast injection of T-61 euthanasia solution.

CT (FD 20-Neuro, Philips Medical Systems) images for each animal were collected before intervention (figure 3.1 panel a: A and used to identify the position of the electrode belt. Realistic tetrahedral mesh of each animal for EIT image reconstruction were generated from the CT images using the approach described in [62]. The ears 
a

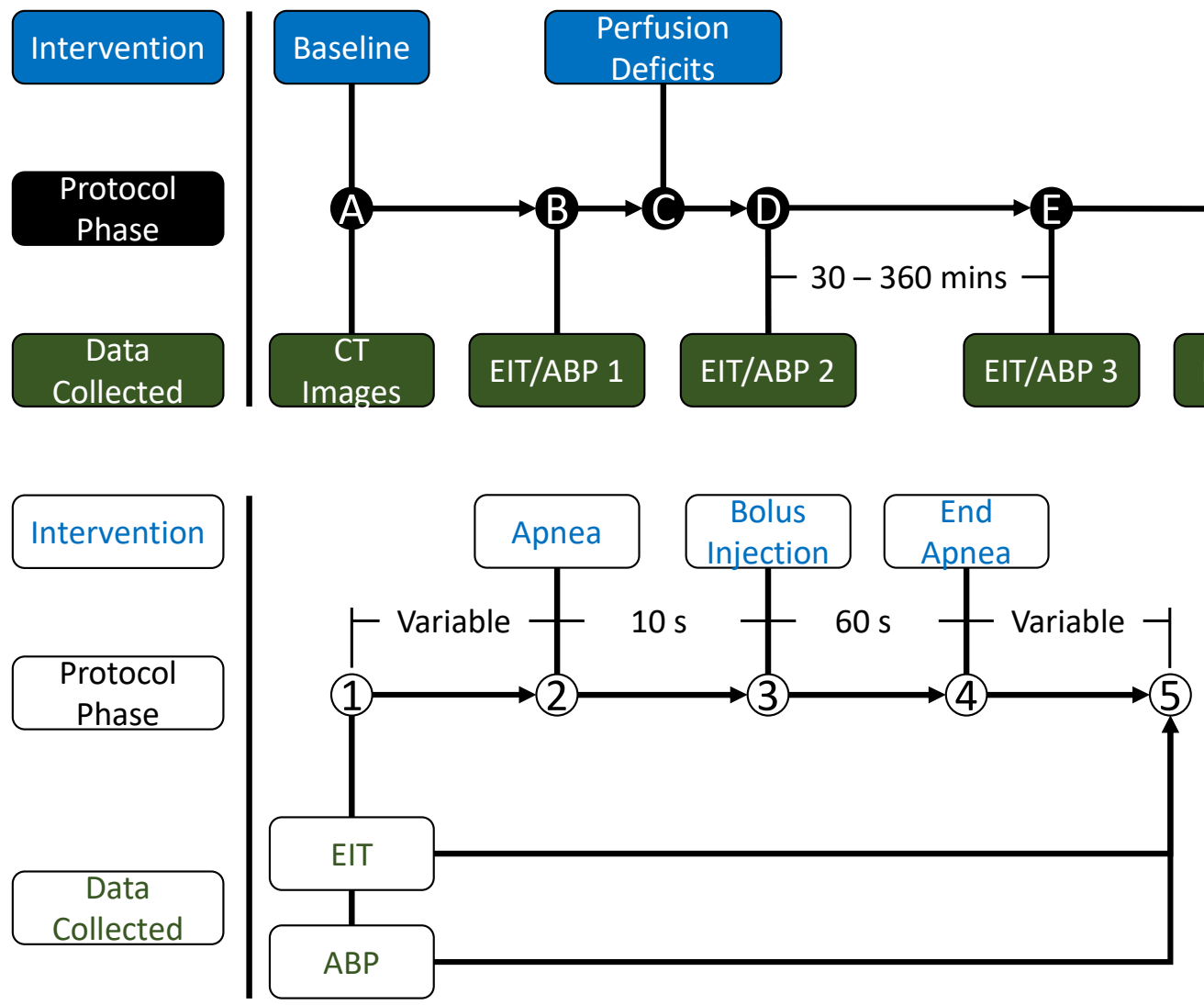

Figure 3.1: Experimental protocol overview. Panel a) Block diagram depicting the overall experimental protocol. A) CT images were collected with the EIT belt attached, during baseline conditions, for locating the electrode plane and for generating reconstruction meshes for each pig. B) EIT and ABP data were then collected at baseline. C) Perfusion deficits were induced in each pig. D) EIT and ABP data were collected again, immediately following perfusion reduction. E) EIT and ABP data were collected a third time, 30-360 minutes after perfusion reduction. F) The EIT belt was removed and MR images were acquired for quantifying ischemia and infarction. Panel b) Block diagram depicting EIT and ABP data collection. 1) EIT and ABP data are acquired simultaneously. 2) Apnea begins after a variable settling period. 3) A saline bolus is injected 10 s later. 4) Ventilation is resumed $60 \mathrm{~s}$ after bolus injection. 5) EIT and ABP data collection are terminated after a variable period.

were manually removed from the images due to the difficulty in meshing the tight angles caused by their folds. The reconstruction plane was defined by the average transverse position of the 4 electrode markers on the EIT belt. CT images were segmented in Seg3D 2.2.1 (University of Utah) into soft tissue, skull, brain, and airway lumen layers, and are shown in figure 3.2. All voxels occupying the skull cavity were assigned to the brain layer. The lumens of airway passages and ear canals 
were assigned to the air segmentation. Meshes were generated using the CGALbased EIT-Mesher meshing tool ${ }^{1}$ and consisted of approximately 1,700,000 elements. Conductivity values for the soft tissue, skull, brain, and airway lumens were set to $0.41,0.016,0.47$, and 0.0001 respectively [24]. EIT images were reconstructed in MATLAB R2019a (The MathWorks, Inc., Natick, Massachusetts, United States) using the GREIT algorithm [20] and the EIDORS software package [63].

The process for creating a brain mask matrix for each pig from the Seg3D model slice of the EIT electrode plane is shown in figure 3.4. Conductivity values for either a region or the full brain were then obtained by setting the region of interest to 1 and all other pixels to 0 , then multiplying reconstructed images by this matrix.
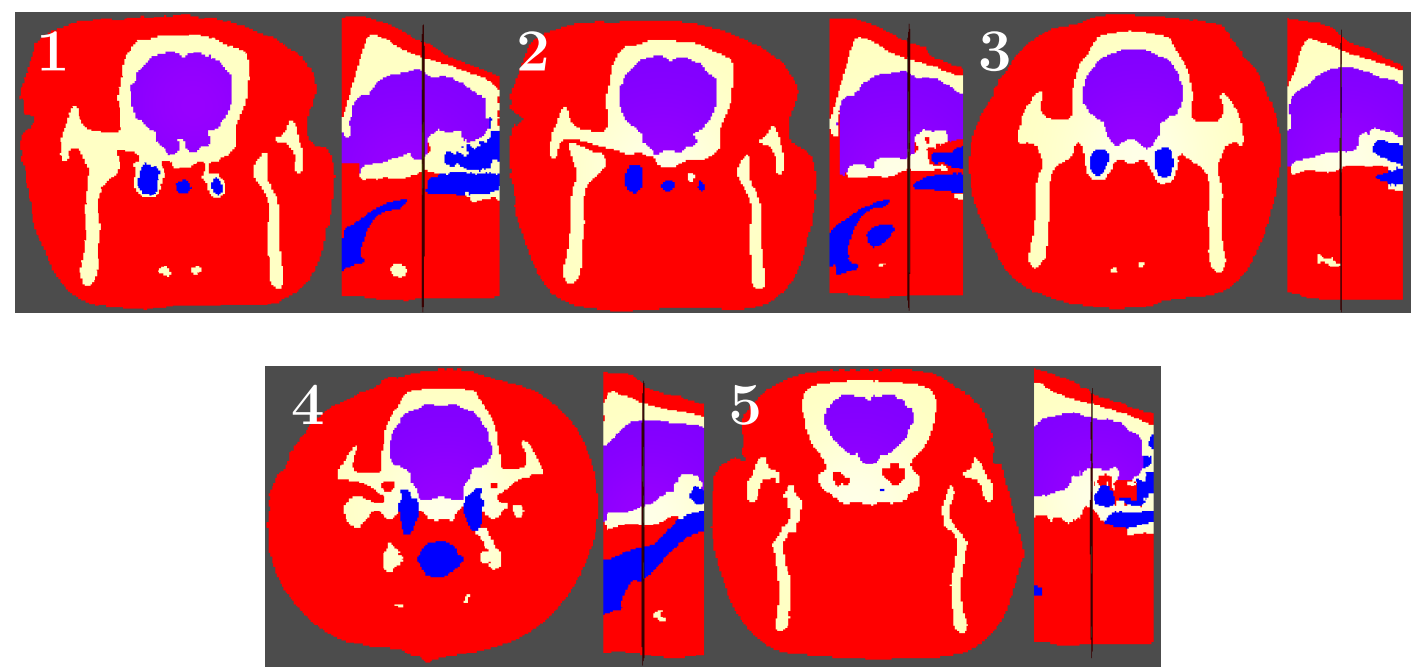

Figure 3.2: Tissue segmentations for soft tissue (red), bone (white), brain (purple), and airway lumens (blue), in anatomical position for animals 1, 2, 3, 4, and 5. Each image shows: left) transverse section showing tissue segmentation in the electrode plane, and right) sagittal section showing the position of the electrode plane with respect to the brain.

EIT measurements were collected from a 32-electrode custom belt (SenTec AG, Landquart Switzerland) and EIT data collection was performed using the SenTec EIT Pioneer Set. Currents were injected at $256 \mathrm{kHz}$ peak-to-peak in a skip-4 stimulation and measurement pattern. The experimental protocol is outlined in Figure 3.1

\footnotetext{
${ }^{1}$ https://github.com/EIT-team/Mesher
} 


\subsubsection{Pre-processing}

Pre-processing and data analysis were carried out in MATLAB. ABP data were downsampled from $1,000 \mathrm{~Hz}$ to $100 \mathrm{~Hz}$, then EIT and ABP sequences were aligned and ABP arterial flush landmarks were converted to the corresponding EIT frame. EIT synchronization spikes were then removed by trimming EIT and ABP data to start one second after the first spike and one second before the last spike. EIT data were then lowpass filtered at $4 \mathrm{~Hz}$ with a $56^{\text {th }}$ order FIR Kaiser window filter. The steep frequency response of this high-order filter was desirable for attenuating noise spikes observed in the Fourier series of several time series which were located between 6 and $10 \mathrm{~Hz}$.

The systolic and diastolic phases of the cardiac cycle were identified by the peaks and valleys in the ABP signal, respectively. ABP data were smoothed with a $15^{\text {th }}$ order moving mean filter, and landmarks were identified with a peak finding function and validated with an in-house peak rejection function. All landmarks within one second of the synchronization flushes were then removed. EIT data were then annotated with the landmarks by converting from ABP to EIT frame number, as shown in figure 3.3 .

Impedance spikes and noise were removed from EIT data using the EQADR algorithm described in chapter 5 .

\section{Ensemble Images}

Ensemble cardiac cycle images were reconstructed from measurements taken before the injection frame. Time periods were adjusted to exclude voltage spikes from electrode detachment when present. Cardiac cycles from the target time period were aligned at systole, then cycles shorter than the maximum length were padded with 
A

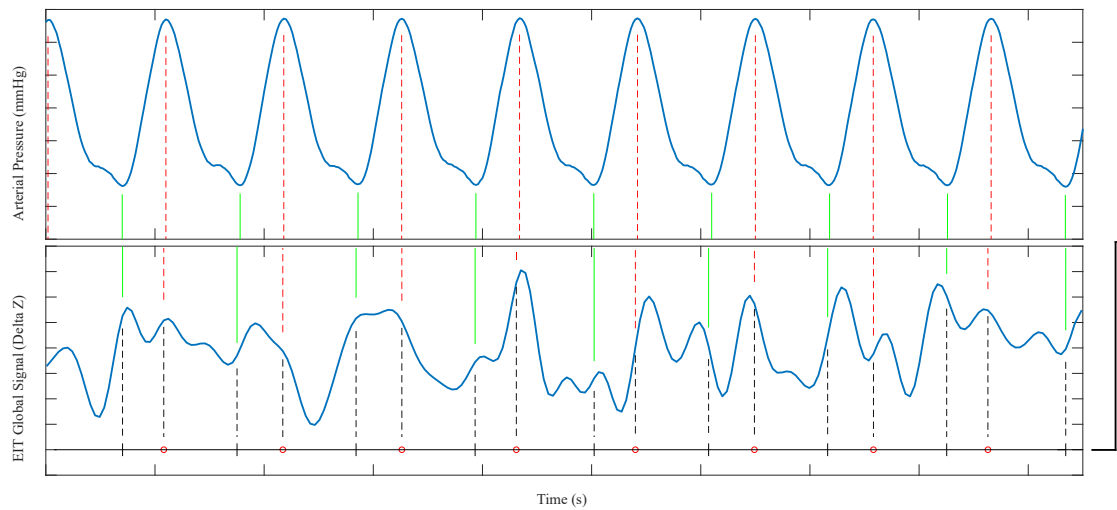

B

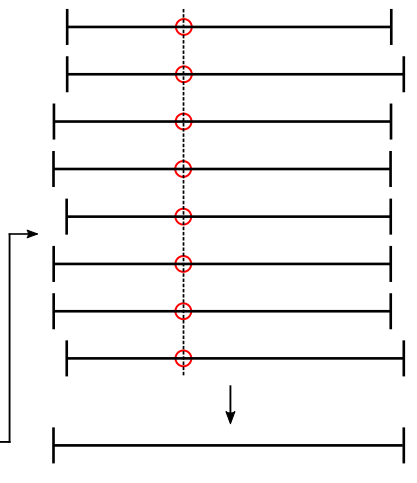

Figure 3.3: Method for producing EIT ensembles using synchronized EIT and ABP data. A) The systolic (red) and diastolic (green) landmarks were located in the ABP data (top) by a peak detection algorithm and transferred to the EIT data (bottom). Diastole-systole-diastole triads were then used to isolate EIT image sequences for each cardiac cycle, shown as the black line in the bottom the EIT data plot. B) The EIT image sequences were then aligned by their systolic landmark and ensemble-averaged to produce a single image series.

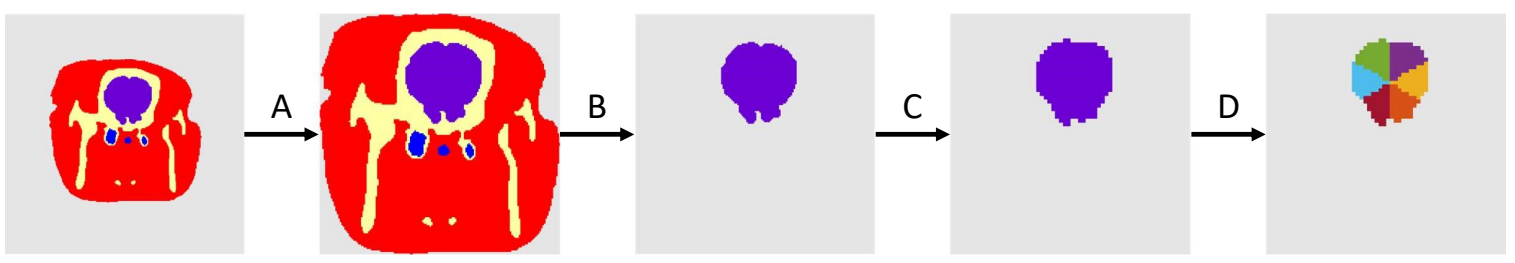

Figure 3.4: Creation of mask matrices for isolating conductivity changes within the brain segmentation. A) Pixels in the segmentation file that were outside of the model's bounding box were cropped. B) All segmentations except the brain were zeroed. C) The image then was resized to the dimensions of the reconstructed images $(64 \times 64$ pixels $)$ and all non-zero pixels were assigned a value of 1 . D) The brain mask was then evenly divided radially into 6 sections using the centroid as the origin. Pixels in each section were assigned the value of their region number (1-6). Conductivity values for a region or the full brain were then obtained by setting the region of interest to 1 and all other pixels to 0 , then multiplying reconstructed images by this matrix.

zero-valued frames on both sides to match the length of the longest cycle. Up to 4 frames were trimmed from the longest cycle if their presentation would have resulted in 6 or more empty frames in the bottom row. The image sets were averaged across cycles by dividing the sum of all images for each frame by the number of non-zero frames composing that frame. Reconstructed conductivity values in the brain segmentation were multiplied by 5 to allow their changes to be clearly seen without being overpowered by the high relative magnitude changes in the soft tissues. 


\section{Saline Bolus Injection}

Saline contrast images were reconstructed using the frame corresponding to the saline injection as the reference. One image per second for 20 seconds after saline contrast bolus injection was reconstructed for each animal. As in the ensemble images, reconstructed conductivity values in the brain segmentation were multiplied by 5 to allow changes in the brain to predominate over changes in the soft tissues to improve interpretation of the images.

\subsection{Results}

In this study, two EIT perfusion-based methods for imaging ischemia with timedifference EIT were developed and tested, using data collected from 5 anesthetized adult pigs (animals 1-5) before, immediately after, and 30-360 minutes after induction of cerebral perfusion deficits [59]. The first and second perfusion-based EIT methods were described in section 3.2.5 and section 3.2.5 respectively.

\subsubsection{Ensemble Average}

The DW-MR apparent diffusion coefficient (ADC) maps and reconstructed EIT images for animals 1-4 are shown in figures 3.5, 3.6, 3.7, 3.8. ADC maps were not available for animal 5, so trace images were used instead, in which perfusion deficits have the inverse signal intensity as in ADC maps. The ADC maps for animal 5, shown in figure 3.9, were therefore shown with inverted colours. Each of these figures begins with three ADC maps taken at protocol phase F (see figure 3.1). The first image is a sagittal section with a green line showing the plane of the two following transverse sections. The transverse sections represent the electrode belt plane as indicated in 
figure 3.2. The second image presents the electrode plane in grey scale, and the third in cold-to-hot - where low and high values are blue and red respectively, as in the EIT images. The reconstructions are presented in the same view as the ADC maps - in anatomical position with the brain at the centre of the section. For clarity, the conductivity values of pixels within the brain segmentation were scaled by a factor of 5 to allow their changes to be seen without being overpowered by the high relative magnitude changes in the soft tissues. The quantification of the extent of ischemia and presence of infarct in these images was performed by a skilled radiologist, however the data was not available at the time of writing but will be included in the submitted version of this manuscript. In their absence, qualitative comparisons between the ADC maps and EIT images were made.

Panels D-F in figures 3.5 - 3.9 show sequences of ensemble-averaged reconstructed images before saline injection from the EIT measurements taken at protocol phases B, $\mathrm{D}$, and $\mathrm{F}$ (figure $3.1 \mathrm{~A}$ ). The first and last frames of the sequence represents average conductivity near diastole. The systolic frame occurs approximately in the middle of each sequence, though due to the difference in sequences lengths used to produce the ensembles and the variable position of systole in each sequence, the diastolic landmarks did not consistently occur at the first and last frames of the ensembles, thus the sequences were not symmetrical. The variable data quality across recordings made for inconsistent numbers of cardiac cycles used for each ensemble image. The number of cycles used to produce each image is shown in table 3.1.

\begin{tabular}{c|ccccc} 
Animal & 1 & 2 & 3 & 4 & 5 \\
\hline Panel D & 5 & 9 & 3 & 5 & 15 \\
\hline Panel E & 17 & 8 & 11 & 31 & 18 \\
\hline Panel F & 8 & - & 28 & 8 & 6 \\
\hline
\end{tabular}

Table 3.1: Number of cardiac cycles used for each animal to produce the ensemble images shown in figures $3.5-3.9$. 
At baseline (figures 3.5 - $3.9 \mathrm{D}$ ), brain conductivity increased primarily on the anatomical right side before systole, followed by the anatomical left side towards end diastole. The high rCBV in this region is in agreement with cerebral blood flow maps of pigs taken with perfusion CT [64]. The highly conductive foci at the superior aspect of the brain in panel $\mathrm{C}$ of figures $3.5,3.6$, and 3.8 are possibly large intra-cerebral venous vessels, that can also be seen in panel $\mathrm{D}$ of figures 3.5, 3.6, and 3.8. Panel $\mathrm{C}$ of figures 3.7 and 3.9 did not show these foci, and the D panels of EIT images accordingly did not show these conductive foci towards systole.

The darker regions in the bottom center and top center of the brain of the ADC maps in figure $3.5 \mathrm{~B}$ and $\mathrm{C}$ indicated a perfusion deficit occurred in these regions. These poorly perfused regions in the bottom of the brain are also visible in the EIT sequences taken after perfusion reduction and 30 minutes after perfusion reduction in this animal. In figure $3.5 \mathrm{E}$, the pattern of perfusion in the brain was altered compared to panel D. At the beginning of the ensemble in E, the base of the brain appeared relatively less conductive than the rest of the brain. The conductivity of the base of the brain on the anatomical right side remained low throughout $\mathrm{E}$ in contrast to $\mathrm{D}$ and suggested that this deficit was detected by the EIT imaging method. This location was again consistently non-conductive in F. The low conductivity appeared washed-out at the end of $\mathrm{F}$ due to the conductivity increase at the base of the brain on the anatomical left side, this may have been due to the flanking conductive foci as indicated by the ADC maps. The top foci in this animal was not detected in the EIT images.

Figure 3.6, panel D for animal 2, showed the putative cerebral vessel in the top left of the brain compared to the top middle in the DW-MR images, which suggested that some image distortion may have occurred. This brain region had low conductivity at both diastoles but peaked towards systole, consistent with the expected conductivity 
changes of a blood vessel over a cardiac cycle. The bottom left area of the brain had low conductivity throughout D, unlike animal 1. This region also had similarly low values in E. The major difference between these image sets was the anatomical bottom right of the brain in E showed consistently reduced conductivity compared to D throughout the image sequence, consistent with the ADC maps, that indicated an area of restricted diffusion (ischemia) on the right side of animal 1.

The ADC maps for animal 3 indicated that an area of ischemia occurred in the bottom left side of the brain figure $3.7 \mathrm{~B}$ and C. The EIT images at baseline showed a conductivity increase in the entire brain, save the top left aspect, towards systole. This indicated that this brain was well-perfused. In E after perfusion reduction, the bottom-left area of the brain showed decreased conductivity relative to $\mathrm{D}$ and indicated a perfusion reduction. The top-middle of the brain also appeared affected in the EIT images, however the ADC maps did not indicate this as an affected area. In F, 210 minutes after perfusion reduction, the anatomical left side of the brain appeared to remain affected in the EIT images, however this region had high values at the beginning of the F) and low values towards the end, though still show reduced conductivity close to the ischemic area compared to D.

The ADC maps for animal 4 suggested that no ischemia occurred in the electrode plane but figure 3.8 A showed an area of restricted diffusion anterior to the electrode plane. The EIT images in D and E followed the same general patterns, with moderate conductivity levels throughout the brain, and a small low-conductivity region in the upper right region of the brain. The images in F suggested that there was a bilateral perfusion reduction in the upper region of the brain, however the ADC maps for the examined plane did not show restricted diffusion in this region.

The trace images for animal 5 (figure $3.9 \mathrm{~B}$ and $\mathrm{C}$ indicated that the brain's bottom and left side were ischemic. The EIT images at D showed mild conductivity 
increases in the center of the sequence towards systole, with the bottom-left and top-left appearing less conductive than the rest of the brain. In contrast to D, after perfusion reduction in figure $3.9 \mathrm{E}$, animal 5 had reduced conductivity in the left and lower-right areas of the brain. While this did reflect the trace images, the presence of the low-valued region in the top-left of the brain in $\mathrm{D}$ reduced confidence in these findings despite the apparent deficit that appeared in a different location in E. After 360 minutes, in F, a deficit was observed on the left side of the brain, though was higher positionally than in E, and appeared more like D than E. Towards the middle of the sequence this conductivity decrease persisted nearly until the end of the sequence unlike $\mathrm{D}$, and was interpreted as indicating perfusion reduction rather than normal signal fluctuation as in D, thus showed moderate agreement with the DW-MR images. 

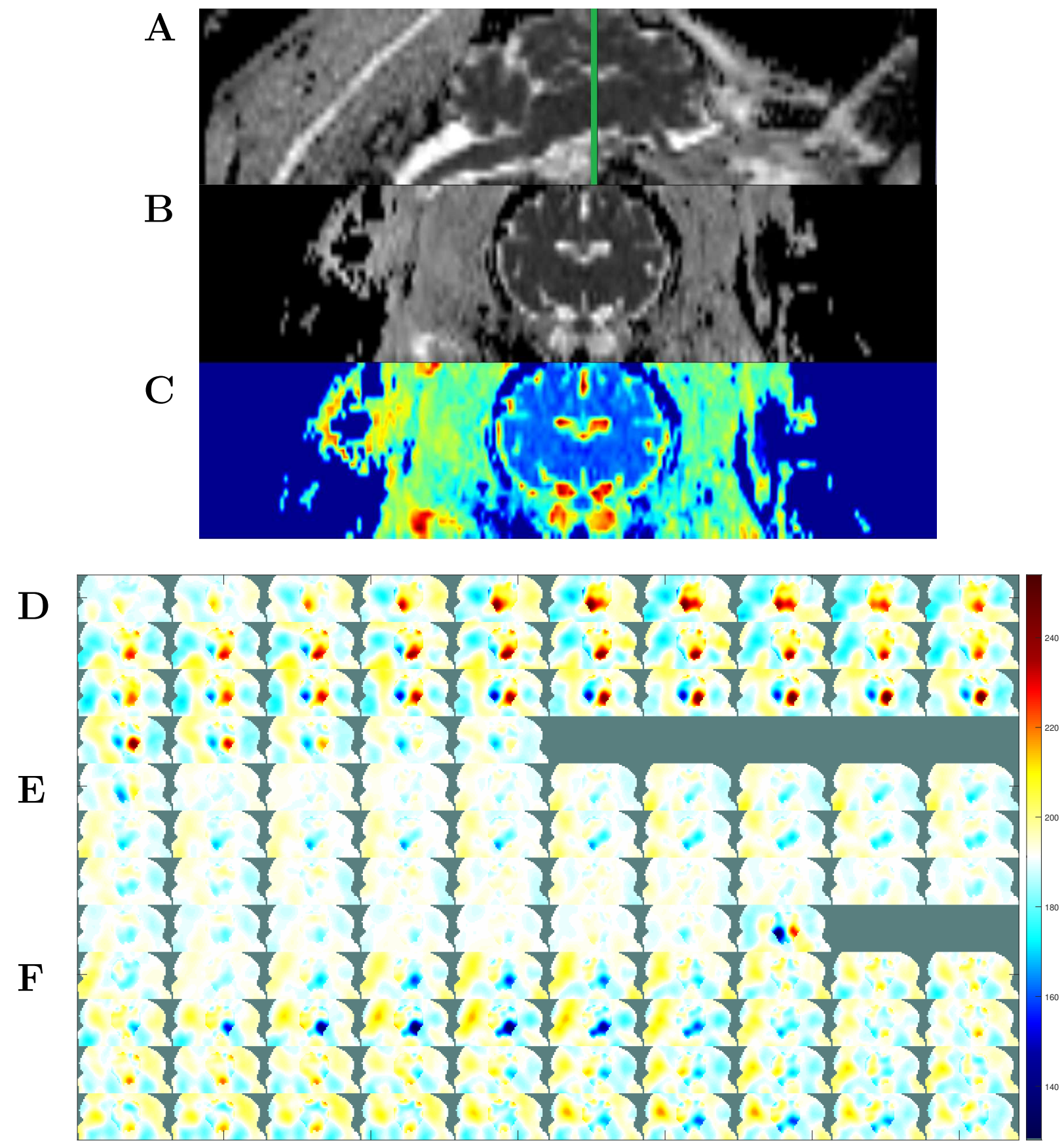

Figure 3.5: DW-MR and ensemble images for animal 1. A) Mid-sagittal section showing the plane of the electrode belt and two following DW-MR images in green. B) Transverse section showing the ep2d-ADC map of the EIT electrode belt plane. C) The same image as in B) but colored cold-to-hot for comparison with the EIT images. D) EIT images at baseline before perfusion reduction. E) Immediately after perfusion reduction. F) 30 minutes after perfusion reduction. Blue represents low conductivity; red represents high conductivity. Colours were scaled independently for each sequence. 

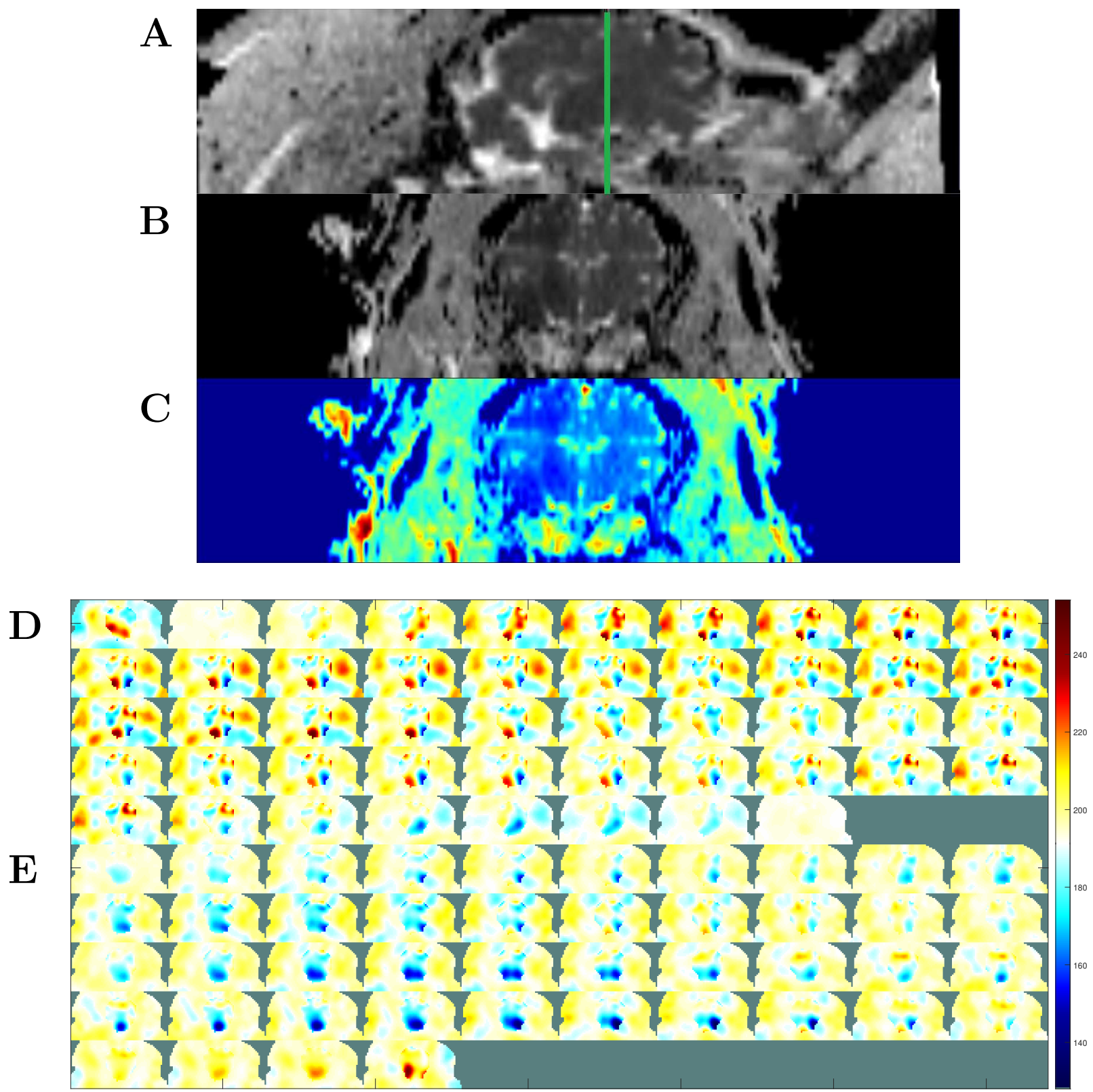

Figure 3.6: DW-MR and ensemble images for animal 2. A) Mid-sagittal section showing the plane of the electrode belt and two following DW-MR images in green. B) Transverse section showing the ep2d-ADC map of the EIT electrode belt plane. C) The same image as in B) but colored coldto-hot for comparison with the EIT images. D) EIT images at baseline before perfusion reduction. E) Immediately after perfusion reduction. Blue represents low conductivity; red represents high conductivity. Colours were scaled independently for each sequence. 

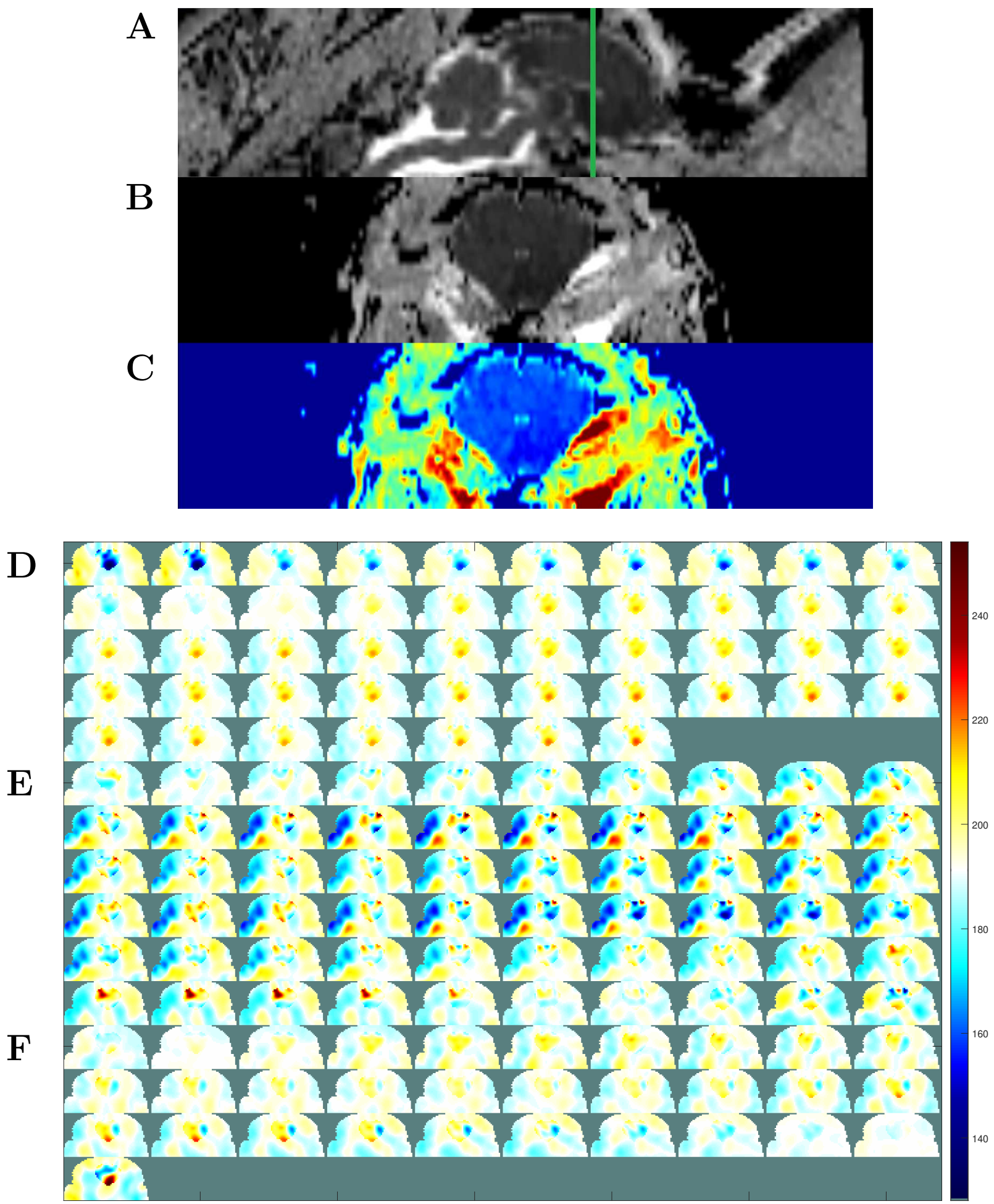

Figure 3.7: DW-MR and ensemble images for animal 3. A) Mid-sagittal section showing the plane of the electrode belt and two following DW-MR images in green. B) Transverse section showing the ep2d-ADC map of the EIT electrode belt plane. C) The same image as in B) but colored cold-to-hot for comparison with the EIT images. D) EIT images at baseline before perfusion reduction. E) Immediately after perfusion reduction. F) 210 minutes after perfusion reduction. Blue represents low conductivity; red represents high conductivity. Colours were scaled independently for each sequence. 

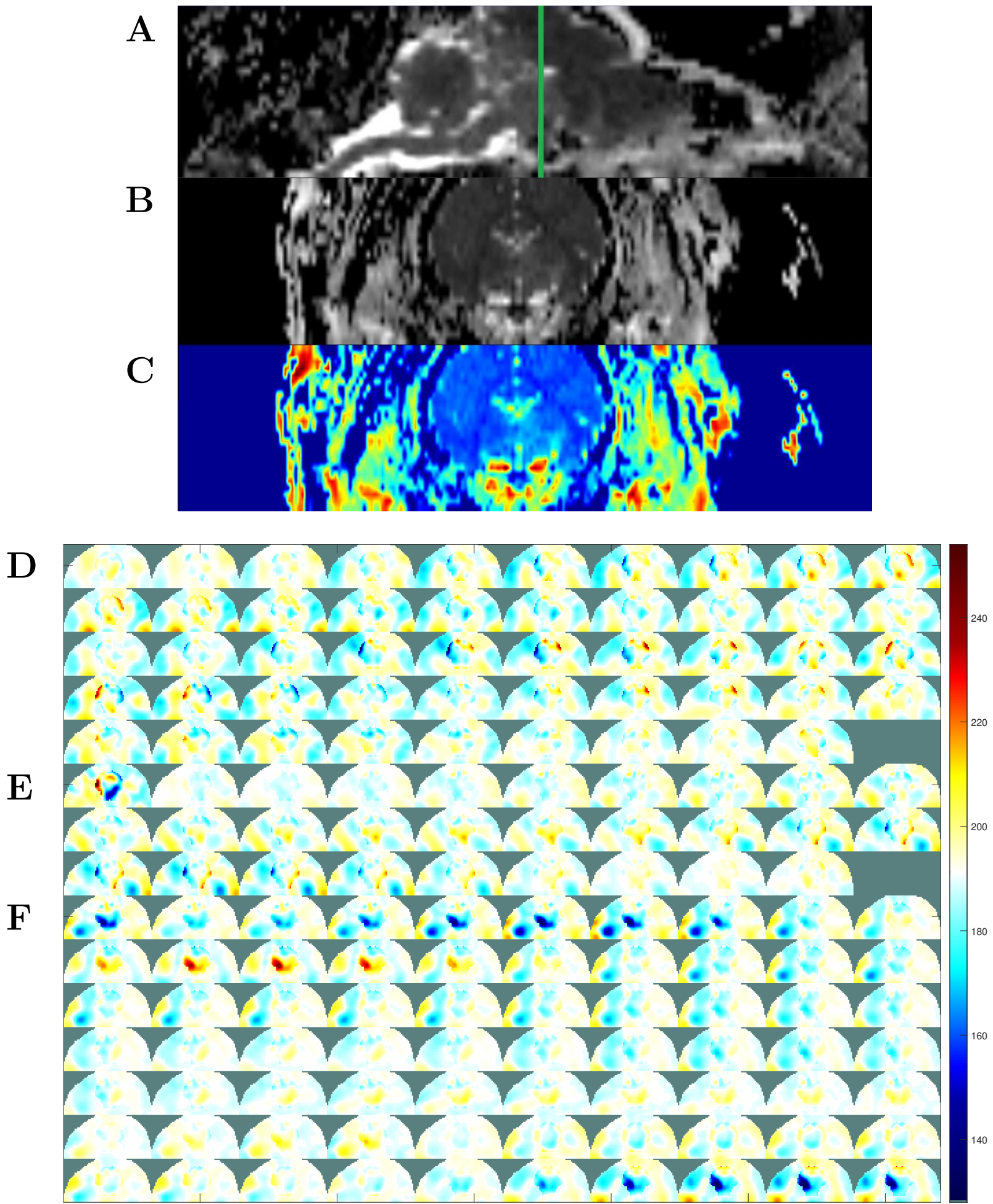

Figure 3.8: DW-MR and ensemble images for animal 4. A) Mid-sagittal section showing the plane of the electrode belt and two following DW-MR images in green. B) Transverse section showing the ep2d-ADC map of the EIT electrode belt plane. C) The same image as in B) but colored cold-to-hot for comparison with the EIT images. D) EIT images at baseline before perfusion reduction. E) Immediately after perfusion reduction. F) 240 minutes after perfusion reduction. Blue represents low conductivity; red represents high conductivity. Colours were scaled independently for each sequence. 

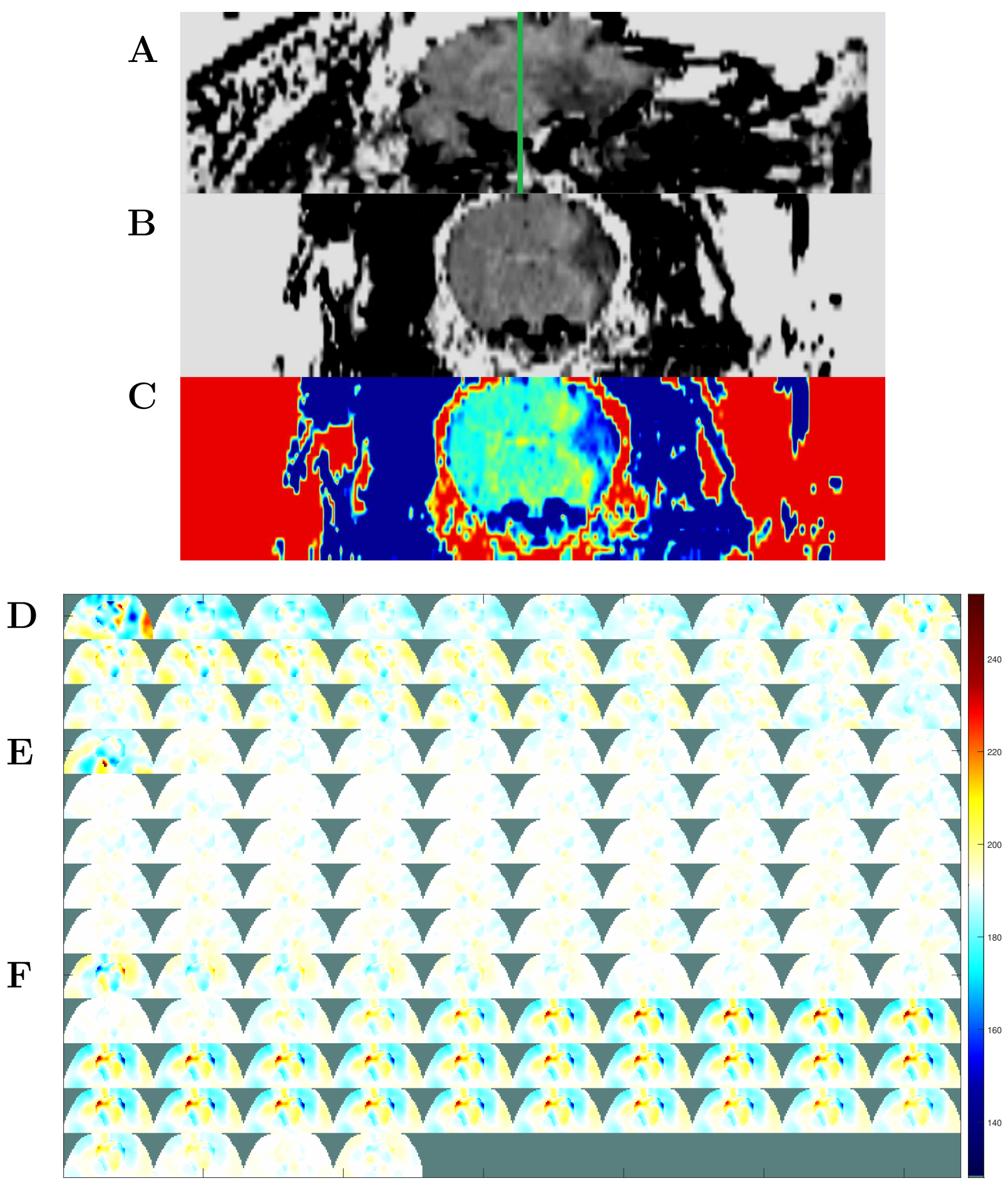

Figure 3.9: DW-MR and ensemble images for animal 5. A) Mid-sagittal section showing the plane of the electrode belt and two following DW-MR images in green. This animal had ep2d-trace but not ADC map, therefore the colour scale was inverted to match the ADC images. B) Transverse section showing the ep2d-trace image of the EIT electrode belt plane with inverted colouring. C) The same image as in B) but colored hot-to-cold for comparison with the EIT images. D) EIT images at baseline before perfusion reduction. E) Immediately after perfusion reduction. F) 360 minutes after perfusion reduction. Blue represents low conductivity; red represents high conductivity. Colours were scaled independently for each sequence. 


\subsubsection{Saline Bolus}

The saline injection images of animal 1 at baseline in figure 3.10 A showed low conductivity throughout the brain for the 20 second period observed, with little change throughout the imaging plane. The differences between $\mathrm{B}$ and $\mathrm{C}$ and $\mathrm{A}$ were not consistent with the perfusion reduction indicated by the ADC maps in figure 3.6 C. The left side of the brain showed consistently higher conductivity than the right for an unknown reason. EIT images for animal 2 at baseline (figure 3.11 A) showed nearly the opposite conductivity trends as in animal 1, with A being high-valued and B after perfusion reduction displaying a highly-conductive region on the right and lower left sides of the brain. The baseline condition of animal 3, shown in figure $3.12 \mathrm{~A}$, was like that of animal 2. After perfusion reduction in figure $3.12 \mathrm{~B}$ the image sequences did not reflect the ADC maps of figure 3.7 C, and figure 3.12 C did not differ from figure 3.12 A. Therefore, the EIT images did not suggest the low-valued region in the base of the brain was due to ischemia. Animal 4 at baseline in figure $3.13 \mathrm{~A}$ showed high-valued regions in the bottom left and right of the brain, which may have matched the corresponding regions in the ADC maps of figure $3.8 \mathrm{C}$, however conductivity changes did not occur throughout the brain as expected in A. Figure 3.12 B showed a conductivity increase in the middle of the left side of the brain and variability on the left side of the brain. Figure 3.12 C showed consistent low conductivity in the base of the brain; however, this did not agree with the ADC maps in figure 3.7 C. The images for animal 5 in figure 3.14 were similar to the ensemble images for this animal, however conductivity in the the left side of the brain was consistently low before and after perfusion reduction, unlike the ensemble images and ADC maps in figure 3.9 . 


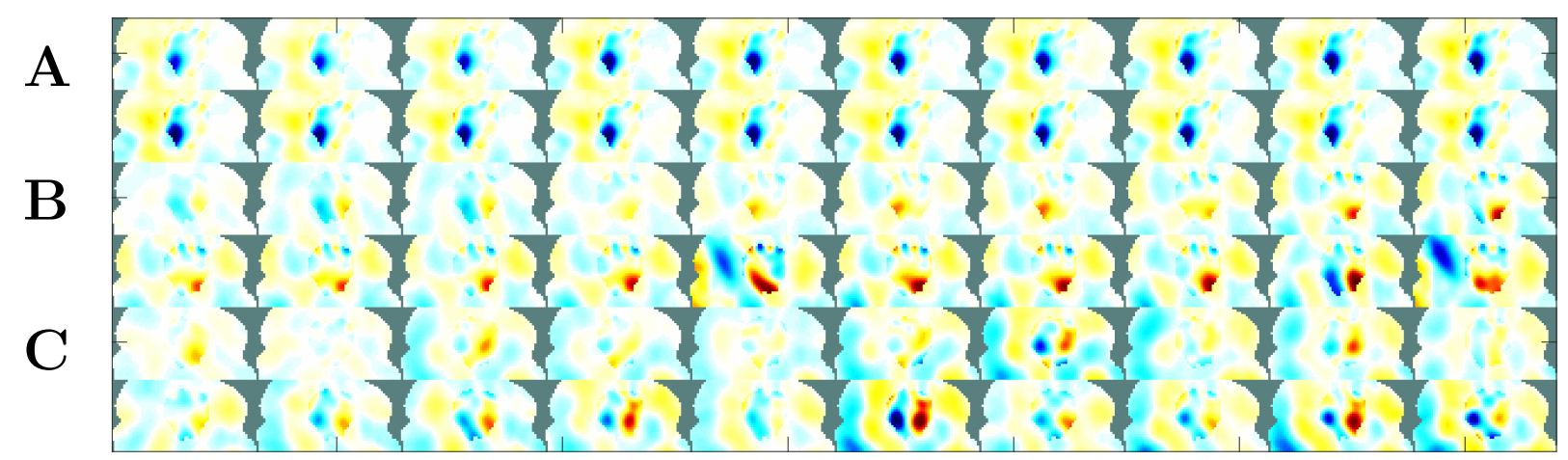

Figure 3.10: Animal 1 saline bolus injection images 1-20 seconds after bolus injection. A) Baseline B) After perfusion reduction C) 30 minutes after perfusion reduction.

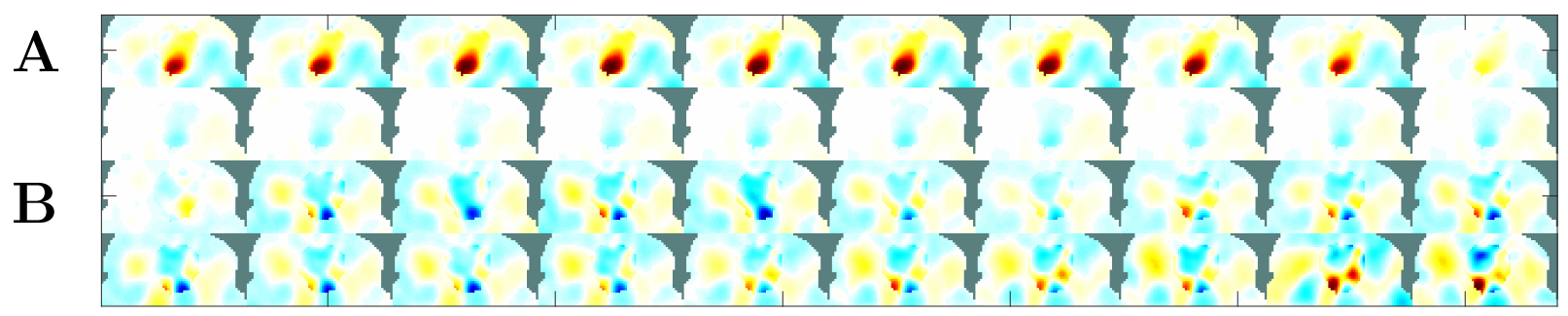

Figure 3.11: Animal 2 saline bolus injection images 1-20 seconds after bolus injection. A) Baseline B) After perfusion reduction

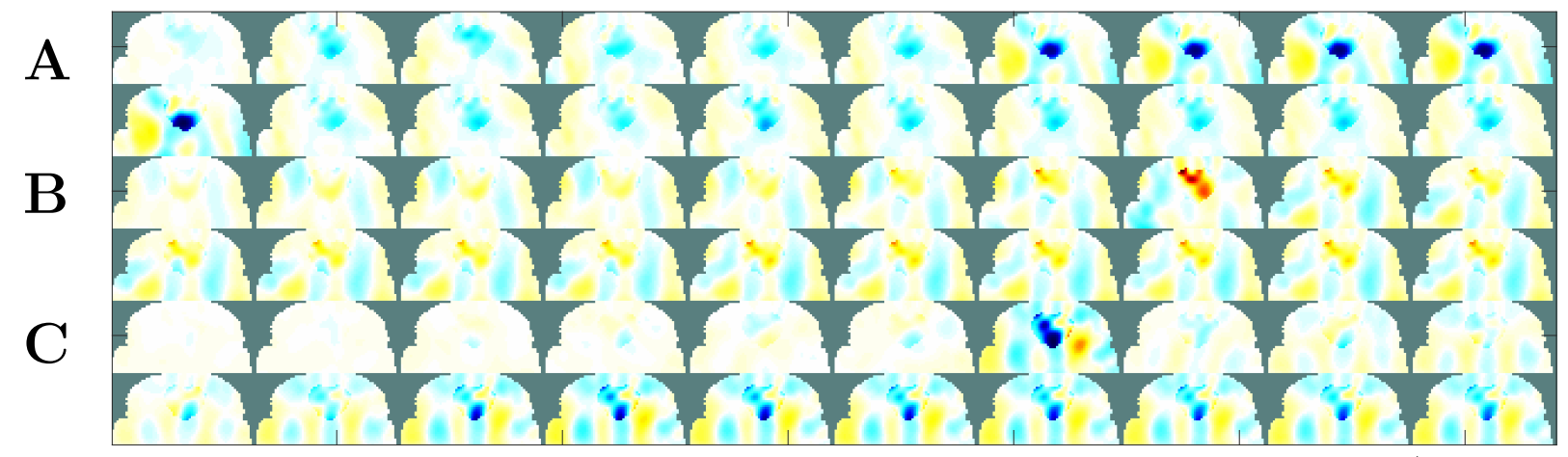

Figure 3.12: Animal 3 saline bolus injection images 1-20 seconds after bolus injection. A) Baseline B) After perfusion reduction C) 210 minutes after perfusion reduction. 


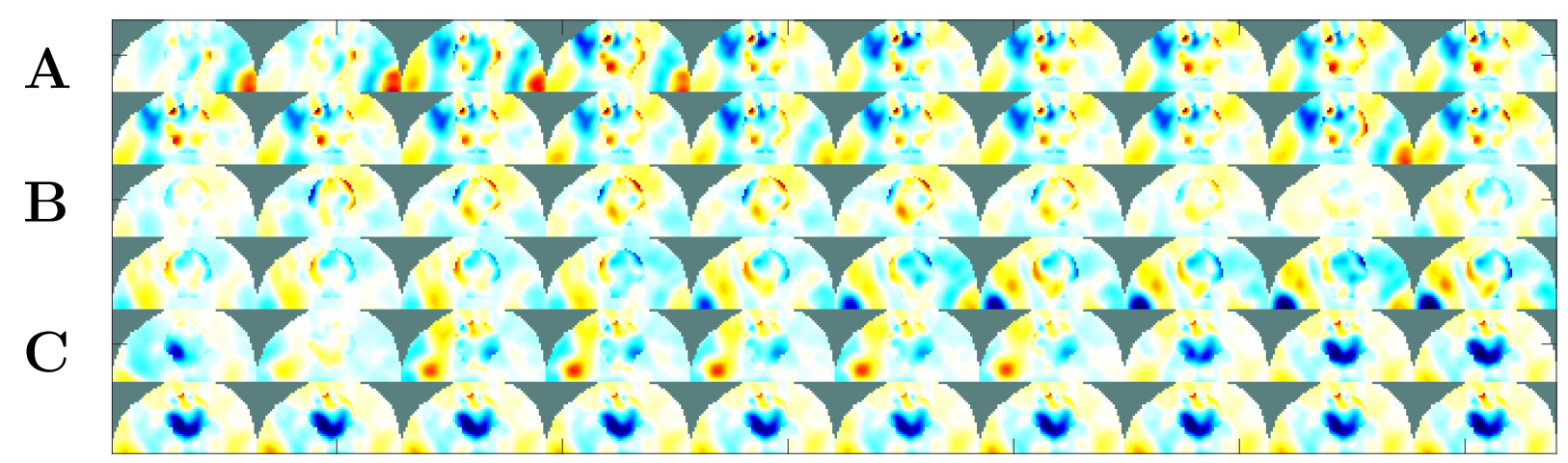

Figure 3.13: Animal 4 saline bolus injection images 1-20 seconds after bolus injection. A) Baseline B) After perfusion reduction C) 240 minutes after perfusion reduction.

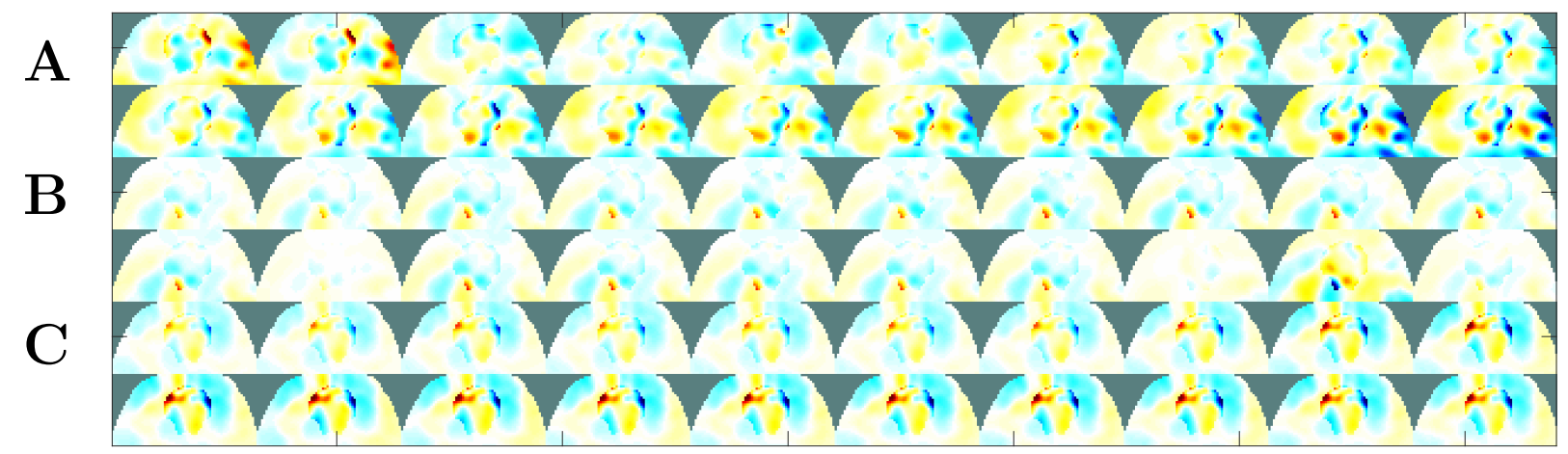

Figure 3.14: Animal 5 saline bolus injection images 1-20 seconds after bolus injection. A) Baseline B) After perfusion reduction C) 360 minutes after perfusion reduction.

\subsection{Discussion}

The goal of this work was to develop perfusion-based EIT-BI techniques for detecting ischemia in an animal model. Of the two novel techniques tested, the ensemble technique was the most successful at detecting ischemia, while the saline bolus injection technique was unsuccessful. The ensemble method detected conductivity decreases after perfusion reduction and at the variable time courses after perfusion reduction that were consistent with areas of restricted diffusion (ischemia) observed by MRI, in $4 / 5$ animals $(1,2$, and 3,5$)$. The saline injection images were not consistent with the DW-MR images for any of the animals. 
The consistent patterns of conductivity change observed in the baseline images for several animals increased confidence that these sequences reflected biological processes and not noise. The first and last image in each ensemble, representing conductivity near diastole, were not consistent in each animal due to the difference in sequences lengths used to produce the ensembles, and the variable position of systole in each sequence (figures 3.5-3.9).

The ensemble images did not reflect the MR images exactly, however in $4 / 5$ animals a consistent conductivity decrease could be seen in the corresponding brain region, that was present after intervention, though not at baseline. Cellular edema caused by cerebral infarct leads to conductivity decreases in the brain [27], and therefore may have contributed to the decreases observed in the EIT data taken at protocol phase E, at the end of the variable time period after perfusion reduction. Evidence that the ensemble technique was able to detect perfusion reduction, and not edema alone, was provided by the results of animals 1, 2, 3, and 5, in the EIT data collected in protocol phase D. In these animals, conductivity decreases were observed in the corresponding regions where restricted diffusion was observed in the ADC maps or trace images, at a time point before cell death and edema should have occurred.

The results of the ensemble technique were contrasted to the saline images, where little consistency was seen across baseline conditions, and conductivity decreases were not observed where DW-MR indicated they had occurred. These discrepancies could be the effect of saline dispersion throughout the circulation, both within and outside of the skull. The higher sensitivity of EIT to conductivity changes near the electrodes relative to the center of the animals causes these external changes to dominate; the intense signal from the saline contrast agent washing out changes occurring in the brain. The pattern of saline dispersion in these animals may follow more closely the pattern of tracer dispersion used in PW-MR images, rather than the diffusion 
coefficients of DW-MR. Thus, while the saline bolus injection method may still hold value, perhaps PW-MRI would have served as a more appropriate reference standard than DW-MRI.

The size and position of induced strokes using PHIL ${ }^{T M}$ were difficult to control due to the large vascular collateralization of the animals. The large strokes observed in animals 1, 2, and 3 therefore may not be representative of the average size of strokes that occur in humans. These animals represent extreme stroke cases and are a limitation of this study.

Since data before saline injection had to be used to producing the ensemble images, the number of epochs that could be used to produce the averaged image was limited by the data quality in between phases 1 and 3 of figure $3.1 \mathrm{~b}$. The results are limited by the number of cardiac cycles that could be used to produce the ensemble images, which are shown in table 3.1. It is expected that approximately 50 epochs, which could be acquired over a 1-minute recording period, would increase the quality and consistency of the ensemble images.

A more detailed analysis and quantitative assessment of the results required quantification of cerebral perfusion deficits and extent of infarct by a trained radiologist, which is currently underway, but was not available at the time of writing. In lieu of these quantitative results, the qualitative analysis of these images indicated that the ensemble technique was capable of detecting these perfusion deficits from surface EIT electrodes in $80 \%$ of the animals, suggesting that this technique may enable non-invasive detection of ischemia in humans. With the skull of a pig being thicker than that of a human and imposing a greater barrier to current flow. Therefore, any consistent changes observed with these EIT techniques in this model, even though the perfusion reductions were severe, is encouraging and suggests these techniques may be equally or more effective in humans for the reasons give. Detection of hemorrhage 
was not tested due to the previous successes in other studies, thus efficacy of this technique for detection of hemorrhage must be tested in future studies. In summary, of the 2 novel perfusion-based EIT-BI imaging techniques developed for analysis of these data, the ensemble method was the most effective and proved to be a viable technique for use in future studies of EIT-BI. 


\section{Chapter 4}

\section{Towards Measuring Brain Function with Perfusion EIT}

\subsection{Purpose}

The goal of this chapter was to create the hardware, reconstruction model, and software tools necessary for translating the animal experiments in 2D from chapter 3 to human experiments in 3D. An EIT electrode suitable for the thick hair covering of the scalp was designed, fabricated, and tested. A realistic human head reconstruction model with 32 electrodes in the international 10-20 placement was designed, created, and tested using simulated data with conductivity perturbations in the range expected to occur in stroke and functional activity. A REB-approved protocol (project \#111259) for collecting EIT measurements of functional activity was developed. A software tool for delivering a randomized n-back working memory task with customizable parameters was created, which included features for synchronizing EIT and ECG data acquisition, and the timing of stimulus presentation, responses, answer correctness, enabling a wide range of ensemble-averaging schemes in post-hoc 
analysis. Unfortunately, the COVID-19 pandemic prevented data collection and purchase of materials for completing the electrode headset, however plans have been made to collect data for this experiment once it is safe to do so.

\subsection{Background}

Biopotential electrodes convert electrochemical potentials, created by ion movement within the body, into electrical potentials, that can then be digitally processed and used for a wide array of healthcare applications. The non-invasive collection of biopotential signals from the brain poses the unique challenge of optimizing the electrode-skin connection through a thick hair covering. $\mathrm{Ag} / \mathrm{AgCl}$ electrodes used with $\mathrm{Ag} / \mathrm{AgCl}$ conductive paste are the standard for EEG montages because of their biocompatibility. The $\mathrm{Ag} / \mathrm{AgCl}$ paste is applied to the scalp through the hair and forms a conductive bridge between the skin and electrode. This type of electrode has been found to be suitable for EIT-BI $[58,19]$. Large montages using these types of electrodes involve long setup and cleanup times, require skilled technicians to apply correctly, and have the potential for short-circuiting between nearby electrodes as the conductive paste settles [65]. On the other hand, polarizable (or "dry") electrodes do not require conductive paste and are of research interest for EIT because they can have low $C_{Z}$, good $C_{Z}$ stability over time, greatly reduced setup times, and remain comfortable throughout long recordings. EIT electrode arrays have the strict requirement for precise electrode spacing and locations, to limit modelling errors and image artifacts, and for low $C_{Z}$, to maximize current flow through the body and minimize polarization potentials. Therefore, an easily donnable headset with polarizable electrodes in a pre-determined configuration, that is self-adapting to different head sizes and shapes, with low $C_{Z}$, would be ideal for EIT-BI. 


\subsubsection{EIT Electrodes}

Numerous electrode designs have been proposed and tested for EIT-BI. Tidswell et al. [57] performed a comparison of 4 headsets with different electrode types, evaluating the measurement noise in human and tank data, ease of use, and image quality of each headset. The electrode types were hydrogel electrodes, saline-soaked sponges, a headcap using electrode gel-filled wells with circular electrodes, and a conventional $\mathrm{Ag} / \mathrm{AgCl}$ electrode cup and gel setup. Their results showed that hydrogel electrodes produced the cleanest signal with low $C_{Z}$ [57]. In all cases skin abrasion was an important step for decreasing electrode $C_{Z}$.

Liao et al. used an array of $17 \mathrm{BeCu}$ spring contact probes, that out-performed the $\mathrm{Ag} / \mathrm{AgCl}$ electrodes in $C_{Z}$ tests on prepared or unprepared skin, from $1 \mathrm{~Hz}-10 \mathrm{kHz}$. Performance at higher frequencies is important because brain EIT stimulation currents are normally at $50 \mathrm{kHz}[5]$. Importantly, the dry electrodes showed $C_{Z}$ stability over a 3-hour period, while the wet electrode $C_{Z}$ increased over time as the gel desiccated. The residues left behind by the dried paste can be irritating to patients and is undesirable [65]. This $C_{Z}$ stability is important for obtaining reliable EIT measurements throughout lengthy recordings and shows the benefit of using polarizable electrodes for EIT.

Liu et al. developed a similar electrode array with 6 spring contact probes, but coated them with Pt nanoparticles (NP) that dramatically increased their surface contact area, as revealed by scanning electron microscopy (SEM) [66]. The Pt NP coating dramatically reduced $C_{Z}$, compared to the untreated electrode, at frequencies lower than $100 \mathrm{~Hz}$ - from an average of $\sim 4.5 \mathrm{k} \Omega$ to an average of $\sim 300 \Omega$. After $100 \mathrm{~Hz}$ the $C_{Z}$ of the treated and untreated electrode converged towards the highest frequency tested $(10 \mathrm{kHz})$. Though the authors did not present the $C_{Z}$ values of the 
untreated electrodes at $10 \mathrm{kHz}$, they showed that at $10 \mathrm{kHz}$ the Pt NP-modified electrodes achieved an average impedance of $\sim 225 \Omega$ versus $\sim 340 \Omega$ of the $\mathrm{Ag} / \mathrm{AgCl}$ electrodes. These results suggested that treating polarizable electrode contact surfaces with Pt NPs can yield lower $C_{Z}$ at frequencies near those used for EIT stimulation than conventional $\mathrm{Ag} / \mathrm{AgCl}$. The largest $C_{Z}$ improvements were seen at sub- $100 \mathrm{~Hz}$ frequencies, which are important for mfEIT and fdEIT stroke imaging, as discussed in section 2.4.1.

\subsubsection{Existing Headset Prototype}

A servo-controlled helmet with electrode contacts made of 316 stainless steel, $10 \mathrm{~mm}$ in diameter was recently developed [28]. This helmet reduced $C_{Z}$ by automated scalp abrasion via electrode rotation. Consistent electrode contact pressure against the scalp was maintained through the action of servos. The authors found that the greatest reduction in $C_{Z}$ was achieved after one rotation of each electrode against the scalp. Subsequent rotations saw little to no reductions in $C_{Z}$. Pyramidal and smooth electrode contacts had similar $C_{Z}$ reductions after the first rotation, and the level of electrode compression affected decrease in $C_{Z}$. Therefore, rotation and compression of electrode against the head can reduce $C_{Z}$ considerably, even with smooth electrode heads, though rough head reduced $C_{Z}$ slightly more than smooth heads with additional rotations. Overall, the author reported that the headset was overly complex and heavy but demonstrated the value of a design in which electrode heads can rotate to abrade the stratus corneum. The helmet design benefitted from fast setup times and gave consistent electrode location and spacing on different subjects. Further research in the design and testing of electrode headset arrays will not only make it easier to conduct EIT-BI research but will help with clinical adoption once the technology has matured. 


\subsection{Methods}

To collect measurements of brain activity with EIT, a data collection system composed of an electrode array, realistic reconstruction model, and test protocol were designed and constructed, to be used with the SenTec EIT Monitor (STEM) Pioneer Set (SenTec) data collection hardware, allowing up to 32 electrodes.

\subsubsection{Design and Fabrication of Electrodes for EIT Measure- ments on Skin with a Thick Hair Covering}

The literature review of EIT electrodes suitable for measurements in the presence of thick hair revealed that hydrogel electrodes and spring-contact probes were good candidates for initial testing. Spring-contact probe electrodes are made with spacing between probes heads to allow hair to pass between probes, which allows the heads to contact the scalp. The saline solution used with hydrogel electrodes forms a liquid conductive bridge between the scalp and electrode, allowing current to be conductive around the hairs instead. Silicon-based hydrogel balls were purchased and tested after absorption of either tap water, salt water, or a 50-50\% (v/v) mixture of di-propylene glycol (DPG) (Chemistry Connection) and Spectra 360 Signa Spray (Parker). The two types of spring contact probes tested were the P125-H (2.4 mm dia. 9-point crown tip) (Uxcell (R)) and the P75-D (1.3 mm dia. round tip) (RC Test Tool Store) shown in figure 4.1. The P125-H probes were tested singly and in an array of 6. The P75-D probes were tested in an array of 19. An experimental electrode consisting of fine brass bristles designed by Tarek Harake (TNH Biosystems) was also tested.

Electrodes were tested on sites without hair such as the forehead and forearm, and on opposite sides of the head through a thick hair covering. All tests on non-hairy sites included a control condition with a conventional $\mathrm{Ag} / \mathrm{AgCl}$. Impedance testing 
was performed with the STEM system at a stimulation frequency of $195 \mathrm{kHz}$ instead of the $50 \mathrm{kHz}$ normally used in brain imaging due to limitations of the system. The maximum impedance value displayed was $1500 \Omega$. Some electrodes exceeded this value when tested at $50 \mathrm{kHz}$ and accurate $C_{Z}$ values could not be obtained. The stimulation frequency was thus increased to $195 \mathrm{kHz}$ where $C_{Z}$ values for all electrodes were within the range of the system. This was deemed appropriate because a change in frequency scaled $C_{Z}$ for all electrodes equally and enabled the experiments to be performed.
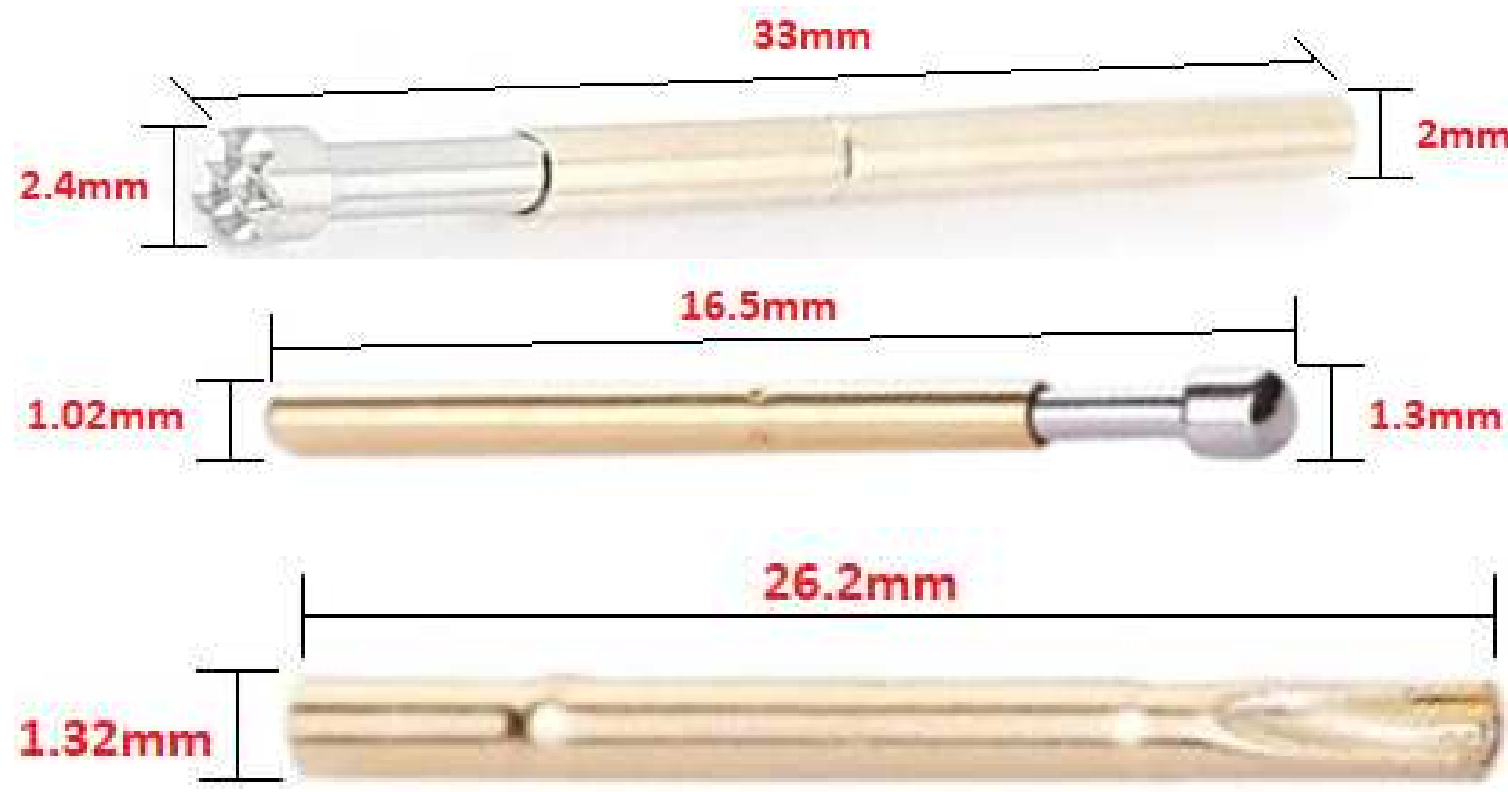

Figure 4.1: Spring contact probe designs. Top: P125-H $2.4 \mathrm{~mm}$ dia. 9-point crown-tipped probe. Middle: P75-D $1.3 \mathrm{~mm}$ dia. round-tipped probe. Bottom: R75-D probe receptacle for P75-D with solder well.

The P75-D electrode array assembly was designed in Fusion 360 (AutoDesk 2019) and fabricated by additive manufacturing. Nineteen R75-D probe receptacles (figure 4.1, bottom) were soldered to $25 \mathrm{~mm} 30 \mathrm{AWG}$ wires with $6 \mathrm{~mm}$ stripped from each end, and inserted into evenly-spaced holes of the spacing grill (figure 4.2, left). The wire connections were tested, then fed through second spacing grill to ensure parallel alignment of the receptacles and even spacing between P75-D contacts once 
inserted into the receptacles. Hot glue was then injected between receptacles to maintain alignment, prevent electrical shorts, and provide mechanical stability for the wire solder connections. The wires from each receptacle were gently twisted together while the glue was hot to provide further mechanical stability to the unit. The ends of the wires were finally trimmed and soldered together. The P75-D probes were then inserted into the receptacles. This design was chosen to allow the use of different probe head types and sizes, or replacing of broken probes, without the need to rebuild the full assembly. The electrode assembly was then inserted into the protective housing (figure 4.2, middle), complete with a retractable exterior mounting ring with pre-drilled holes that can be sewn to a fabric or neoprene cap. The mechanism for securing the electrode housing to the mounting ring and maintaining pressure has not been implemented on this electrode but was planned to be a Velcro ${ }^{\mathrm{TM}}$ strap passing over the housing. The outgoing wire from each electrode was intended to be soldered to the male end of a snap fastener to connect with the electrode leads of the STEM system. The right side of figure 4.2 demonstrates how the probe array maintains contact on uneven surfaces. The spacing between probes was optimized to maintain a small overall electrode diameter of $1 \mathrm{~mm}$, while being large enough to allow the skin to deflect around each probe head, thereby increasing the overall surface area of contact between the probe heads and the skin.

\subsubsection{Creating a Realistic Head Model from Paired CT-MRI Images}

Previous attempts at EIT brain imaging have demonstrated the requirement for a realistic head model to improve image quality [55]. The approach used here to generate the realistic human head tetrahedral mesh has been previously described by 

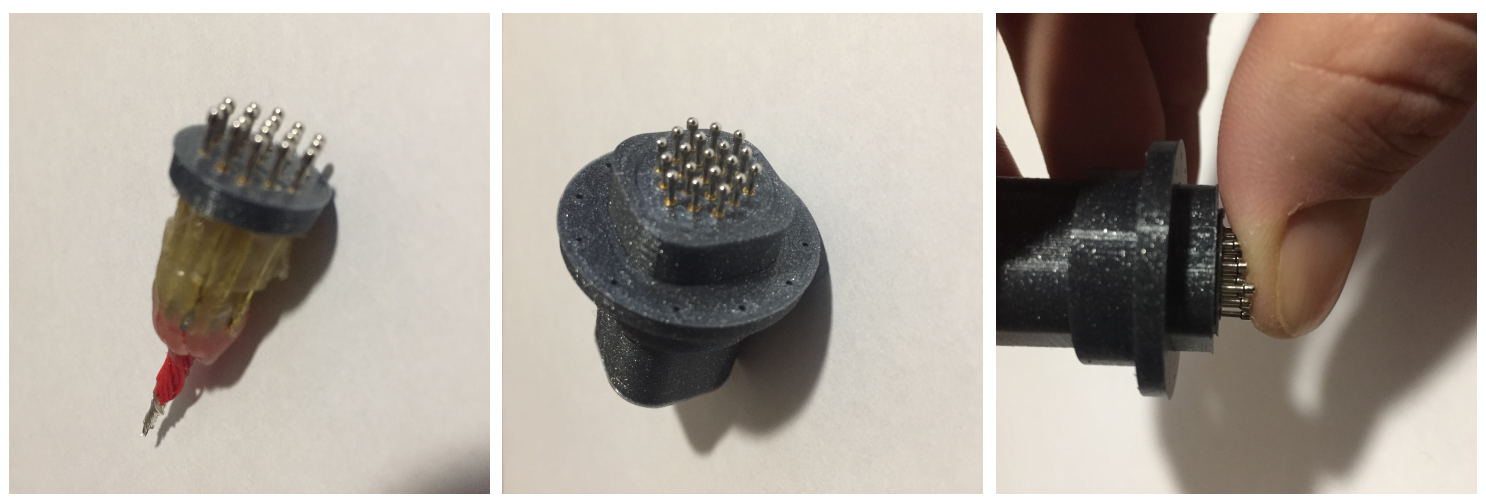

Figure 4.2: Left: Probe array without casing showing internal electronics. Middle: Probe array without casing showing the mounting assembly. Right: Demonstration of probe array spring loading and contact quality on uneven surfaces.

Jehl et al. [62]. Attempts to reproduce the CGAL-based meshing tool used by their group, and to produce satisfactory meshes using Netgen and Gmsh meshing tools were unsuccessful. Fortunately, the UCL group published their mesher on Github ${ }^{1}$ and their mesher could be used directly.

The CT and proton density weighted MRI images used for creating the human head mesh were the 15-year old "brain example" data included with Seg3D (version 2.2.1). This individual had a large break in their skull's left side that was removed by mirroring segmentations of the right side about the mid-sagittal plane.

The spherical coordinates for electrode centres in a 32-electrode montage, according to the international 10-20 system, were obtained from the BioSemi website ${ }^{2}$. The spherical coordinates assumed that the nasion $(\mathrm{Nz})$ - inion $(\mathrm{Iz})$ line fell on the y axis, the right (PAR) and left (PAL) pre-auriculars fell on the x-axis, and the superiorinferior line normal to the plane made by the Nz-Iz and PAR-PAL lay on the z-axis (shown as Nasion-Inion, s-m, and a-o axes in figure 4.4). The transformation matrix mapping the Cartesian axes to the axes of the model was calculated by first locating the Nz, Iz, PAR, and PAL positions on the model, then finding the spherical coordi-

\footnotetext{
${ }^{1}$ https://github.com/EIT-team/Mesher [accessed: 2020-04-07]

${ }^{2}$ https://www.biosemi.com/download.htm [accessed: 2019-08-16]
} 


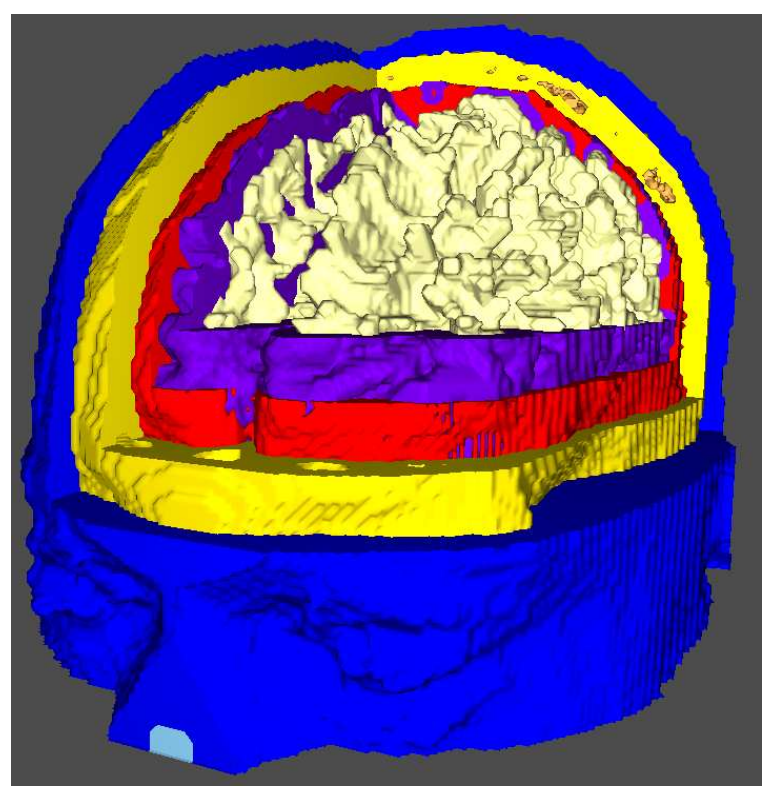

Figure 4.3: 3D cutaway of the segmented human head. Tissue types are presented as follows: dark blue: soft tissue, yellow: bone, red: CSF, purple: gray matter, white: white matter, orange: diploe, and light blue: airway lumens.

nate origin $o$ in model space as the point on the superior-inferior axis, normal to the Nz-Iz and PAR-PAL axes, that formed a $65^{\circ}$ angle between the Iz with respect to the superior-inferior axis, as per Huang et al. [67]. The spherical coordinates were vectorized and oriented in model space via multiplication by the transformation matrix. The electrode centres on the model's surface were then located by the intersection of rays projected from $o$, at the transformed vectors, and the model's surface.

The mesh was made using the UCL mesher, as described in chapter 2 and in [62]. Mesh elements were refined according to their distance from electrodes, with smaller elements closer to the electrodes and larger elements in the centre of the model. This element sizing is important because it reflects the inverse relationship between EIT sensitivity and distance of conductivity changes from the electrodes. Element edge sizes were elements were $1 \mathrm{~mm}$ at the electrodes. Outside of the electrodes, element edge sizes were $4 \mathrm{~mm}$ at their finest and increased to $8 \mathrm{~mm}$ in the model centre. The mesh, shown in figure 4.5 with its individual segmentations, consisted of a total of 


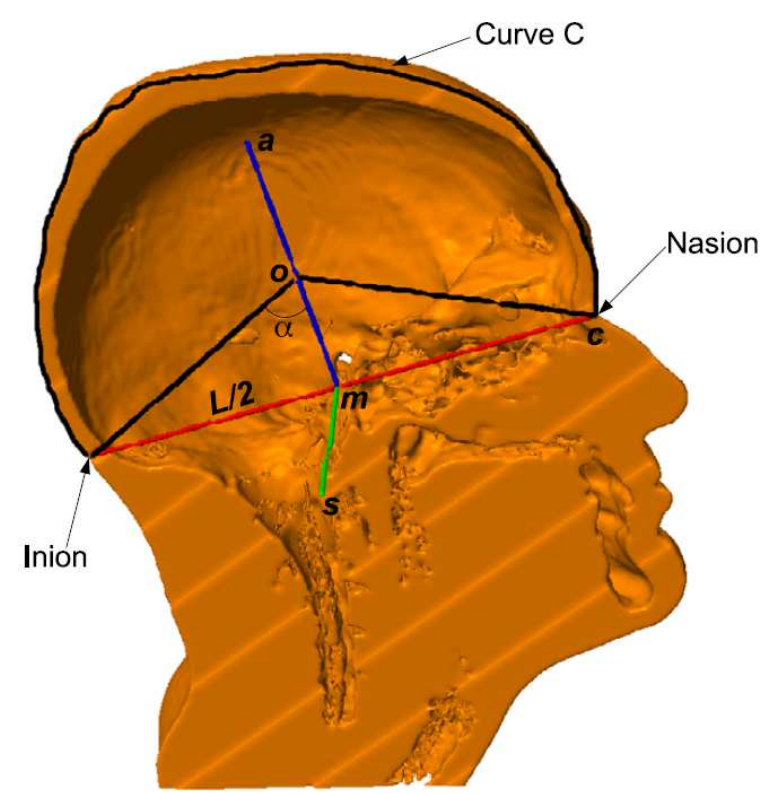

Figure 4.4: Three basis vectors for electrode placement on the human head model. Reproduced from Huang et al. [67], figure 5.

$3,688,836$ elements, with the average Delaunay triangulation quality (ratio of each simplex's circumradius to its shortest edge length) of 0.72 .

\subsubsection{Reconstructions From Simulated Data}

Simulations were performed on the human head reconstruction model to study its sensitivity and localization accuracy for conductivity perturbations typical of stroke and functional activity. Spherical inclusions of three sizes $(1 / 16,1 / 8$, and $1 / 4$ of the average brain radius) were placed at three distances $(1 / 8,1 / 4$, and $1 / 2$ of the average brain radius) from the brain centre at 8 radial positions, using 4 conductivity changes (increases or decreases of $1 \%$ and 10\%). Each image was reconstructed from the average of $1080 \mathrm{~dB}$ SNR measurements. Data was reconstructed on a separate model with $32 \times 26 \times 32$ outside dimensions and cube-shaped elements. 


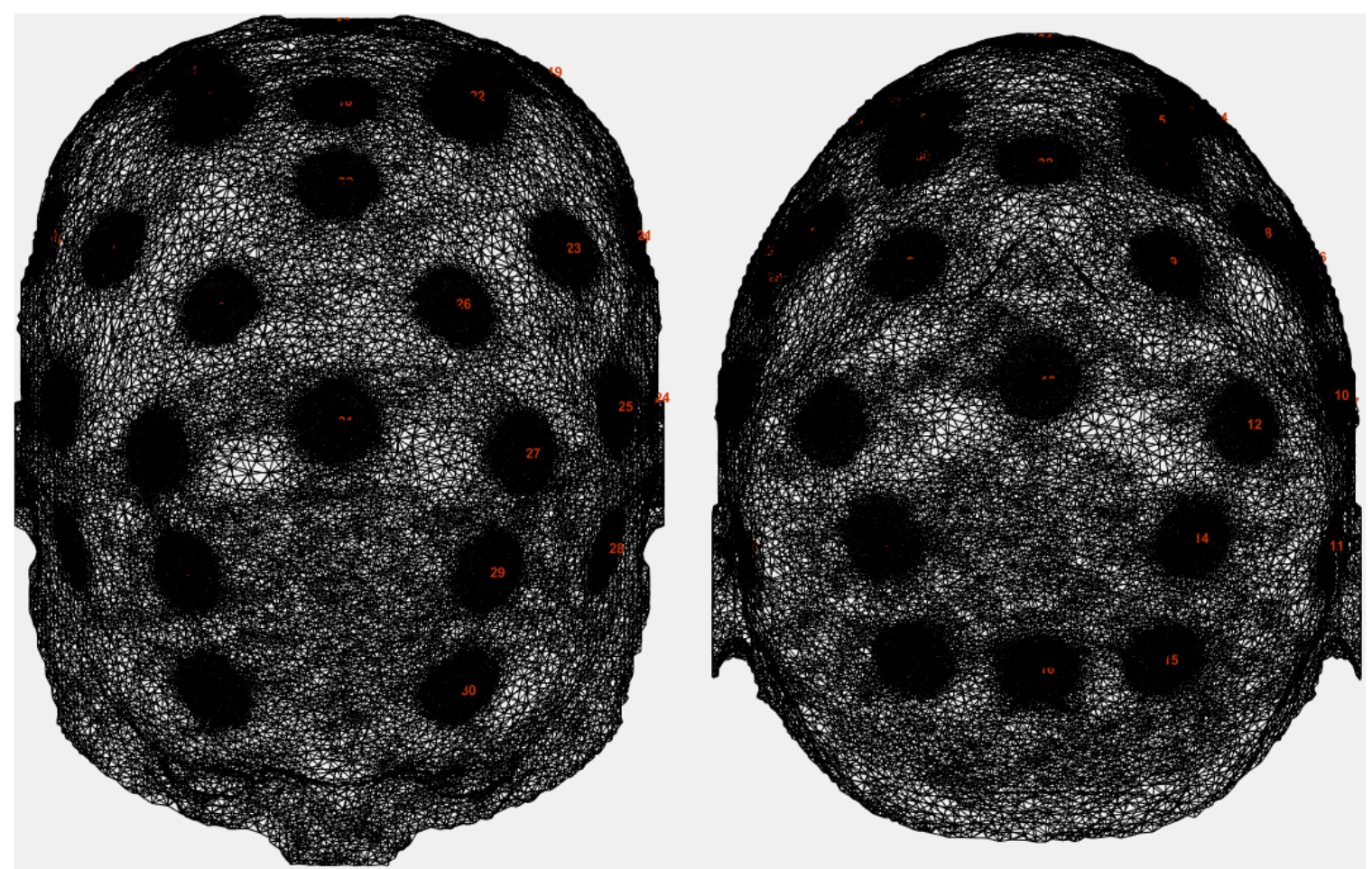

Figure 4.5: The human head finite element mesh, with electrode locations (centers of dense meshing) and numbering (red). Left: Front view. Right: Rear view

\subsubsection{Protocol Design}

Given that CBV changes in functional activity are close to the limit of detection with EIT, an experimental task that allowed averaging of up to 100 stimuli, while having a reasonable time duration, was desired. To increase the chances of detecting activity, a task that required significant mental effort and neural activation, thus a large HDR, was also desired. The activated brain regions during this task needed to be consistent across individuals and well-established for referencing with EIT images. Finally, the advantage of EIT over EEG as a portable, electrode-based functional imaging tool is its ability to image internal and external brain structures, whereas EEG only measures surface activity. The task selected for this protocol therefore needed to activate internal brain regions. The n-back working memory task was selected for its short stimulus-response times, allowing 100 stimuli to be presented 
within an hour-long protocol. The difficulty of the task was expected to require large neural recruitment. Additionally, verbal stimuli in the form of English letters were selected because they produce the largest changes in the brain compared to nonverbal stimuli such as images or numbers [68]. Lastly, the interior Brodmann area 32 is activated in the n-back working memory task, along with surface Brodmann areas 6-10, 44, 46, and 47 [68].

\subsubsection{N-Back Graphical User Interface (GUI) Software}

The n-back protocol delivery GUI was built using MATLAB 2019a. It presents the n-back working memory task to the user, while coordinating synchronized EIT and ECG data collection. The software first presents a welcome screen, shown in the top of figure 4.6. The bottom-right of the GUI contains three drop-down menus for selection of three test parameters. The value of " $n$ " can be set from $1-3$. The length of the test can be up to 100 stimuli (letters), and the number of correct responses can be selected, but caps at (number of stimuli - 5). During the task, the user responds to the stimulus by clicking the "Match" (hotkey: m) button if the letter being shown is the same as the letter shown "n" times ago, or the "No Match" (hotkey: n) button, otherwise. Clicking "Start", brings the user to a screen that explains the protocol (figure 4.6, bottom left). Pressing "Start" again begins the protocol. An example stimulus is shown in the bottom right of figure 4.6. A fixation cross is presented in between each stimulus. The user can change their response until the next fixation

cross is shown. The user may elect to pause or end the test at any time by pressing the "Pause" and "End Test" buttons, respectively.

Upon the first click of the "Start" button, the protocol background processes construct a new random n-back deck from the user's parameters, open the STEM software, and creates a new folder with the participant number and date of data col- 
lection. The second click of "Start" initiates EIT and ECG data collection. Throughout the protocol, the timing of stimulus presentation and user responses are recorded and saved when the user presses the "End Test" button, or after the last stimulus is presented. This data and metadata can then be used for post-hoc analysis of task related CBV changes though ensemble averaging of evoked responses (stimulus presentation or motor response), or ECG pulsatility.

\subsection{Results}

\subsubsection{Electrode Tests}

The $C_{Z}$ of 6 different types of electrodes were tested on one subject at $195 \mathrm{kHz}$ on the exposed skin of the forehead, on opposite sides of the head through the hair, or on either side of the forearm. The results from each electrode design, and a conventional $\mathrm{Ag} / \mathrm{AgCl}$ adhesive electrode control, are presented in table 4.1.

Table 4.1: Contact Impedances of EIT electrodes tested on the head and forearm, with and without ultrasound gel.

\begin{tabular}{|c|c|c|c|c|}
\hline Electrode & Location & Hair? & Gel? & Impedance@ $195 \mathrm{kHz}(\Omega)$ \\
\hline $\mathrm{Ag} / \mathrm{AgCl}$ & Forehead & No & No & 400 \\
\hline $\mathrm{Ag} / \mathrm{AgCl}$ & Forearm & No & No & 350 \\
\hline P125-H array (6) & Opposite sides of head & Yes & Yes & 1000 \\
\hline P125-H array (13) & Opposite sides of head & Yes & Yes & 700 \\
\hline P75-D array (19) & Opposite sides of head & Yes & Yes & 750 \\
\hline P75-D array (19) & Forehead & No & Yes & 475 \\
\hline P75-D array (19) & Forearm & No & No & 525 \\
\hline P75-D array (19) & Forearm & No & Yes & 350 \\
\hline Hydrogel + water & Forehead & No & No & 750 \\
\hline Hydrogel + saline & Forehead & No & No & 700 \\
\hline Hydrogel + 50/50 DGP/Signaspray & Forehead & No & No & 700 \\
\hline Brass brush & Opposite sides of head, pressing into scalp & Yes & No & 820 \\
\hline Brass brush & Opposite sides of head, pressing into scalp & Yes & Yes & 620 \\
\hline Brass brush & Opposite sides of head, gentle pressure & Yes & Yes & 900 \\
\hline Brass brush & Forehead & No & Yes & 700 \\
\hline
\end{tabular}

$\mathrm{Ag} / \mathrm{AgCl}$ did not use ultrasound gel but had conductive electrolyte.

Using ultrasound gel decreased $C_{Z}$ for the P125-H and P75-D arrays, and the brass brush electrodes in all cases, except when the brass brush electrodes were applied with 


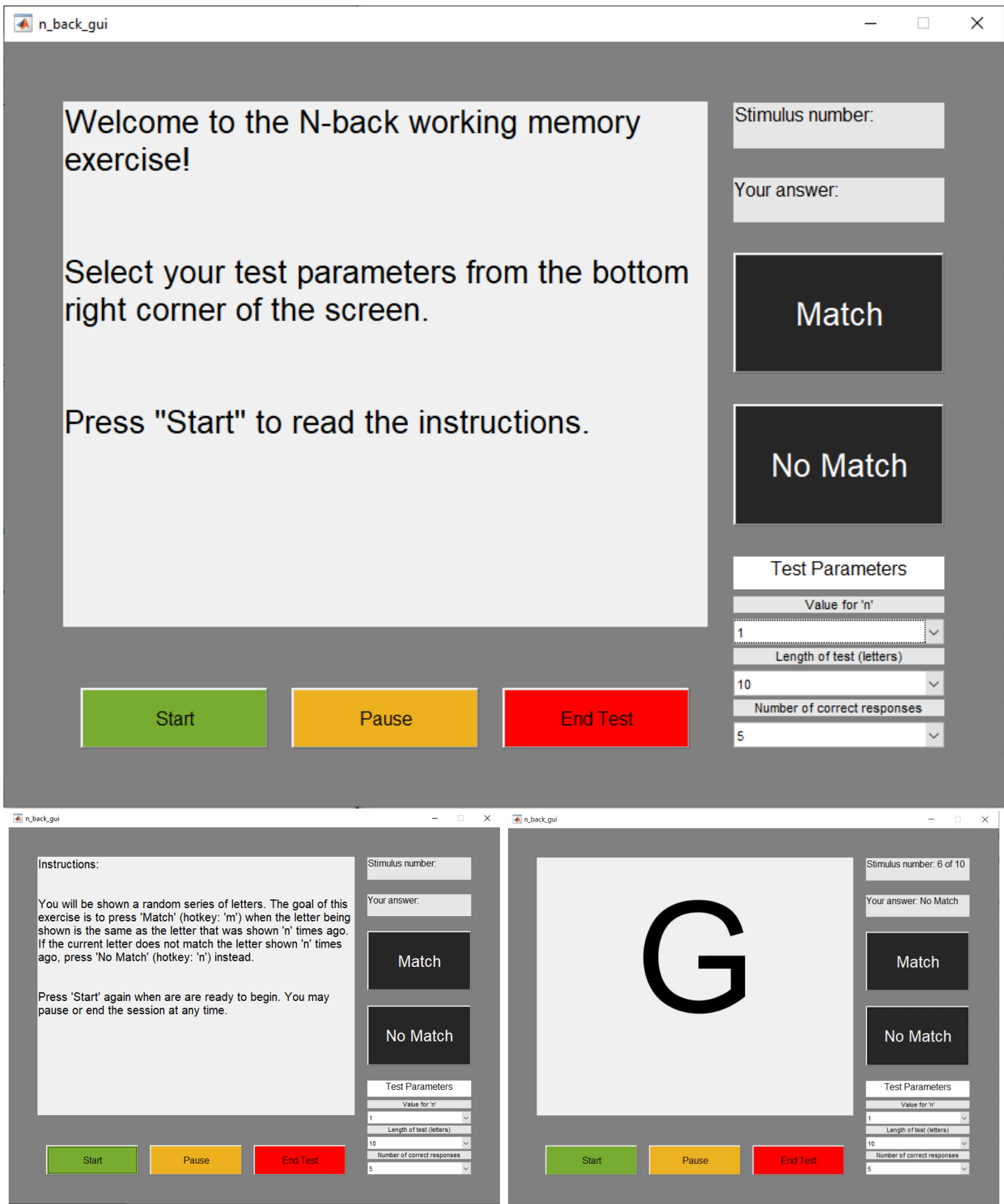

Figure 4.6: Presentation of the N-Back GUI Software. Top: Welcome screen. Bottom left: An instruction screen is shown after the user presses "Start" for the first time. Pressing "Start" again displays a countdown until the task begins. Bottom right: A sample stimulus is shown in the main window. The window in the top right of the interface displays how far the user is into the task. Just below, their current response is shown. The user may change their response until the next fixation cross is shown. 
gentle pressure. Increasing the number of probes in the $\mathrm{P} 125-\mathrm{H}$ array decreased the $C_{Z}$. The brass brush electrodes with forceful application achieved the lowest $C_{Z}$ when applied on opposite sides of the head with ultrasound gel but were painful. The 13-pin P125-H array achieved the lowest $C_{Z}$ of $700 \Omega$ while also being comfortable on the scalp. The 19-pin P75-D array achieved the second-lowest $C_{Z}$ of the tested electrodes, at $750 \Omega$. The smaller shaft diameter and shorter overall length of the P75-D probe compared to the P125-H probe allows it to achieve a smaller overall electrode size and was the preferred option. The difference in $C_{Z}$ between the standard $\mathrm{Ag} / \mathrm{AgCl}$ electrodes and the 19-pin P75-D array with electrode gel on the forehead was $75 \Omega$. The hydrogel electrodes achieved much higher $C_{Z}$ than the 19-pin P75-D array and $\mathrm{Ag} / \mathrm{AgCl}$ electrodes on the forehead. They performed as well as the brass brush electrodes on the forehead, while having the benefit of comfort.

\subsubsection{Simulations}

Figures 4.7 and 4.8 show the localization error in the $\mathrm{x}-\mathrm{y}$ plane for the simulations as a function of inclusion radial position. The localization error was lowest for the small inclusions and highest for the large inclusions. Localization error in the z-plane was affected by the portion of the model below the electrode plane, in which the spherical inclusions were projected as cones or cylinders, which made the z-plane localization error large. The localization error for all inclusion sizes was the largest at the -0.79 and 0 radial positions and the lowest at the -3.14 and 2.36 radian positions. Figures 4.9 and 4.10 showed reconstructions from simulations of a $10 \%$ increase and decrease respectively. The presence of spurious artifacts may have led to these poor localization accuracies, as the centre of gravity did not change as much as expected for different inclusion positions. Thus, the localization accuracies decreased as the inclusion centre rotated farther away from the relatively stationary centre of gravity, 
and vice versa.

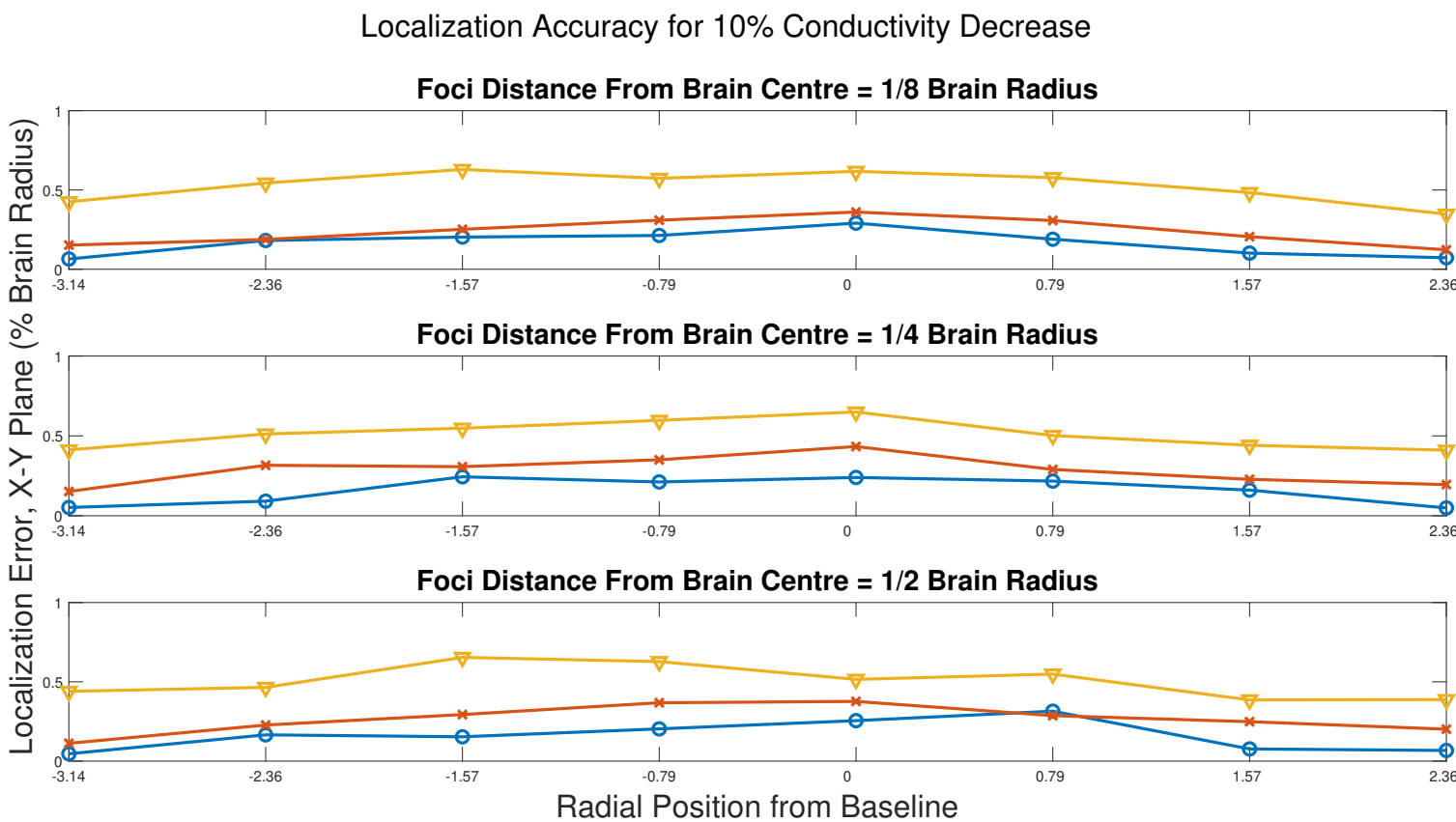

Localization Accuracy for $10 \%$ Conductivity Increase

Foci Distance From Brain Centre $=1 / 8$ Brain Radius

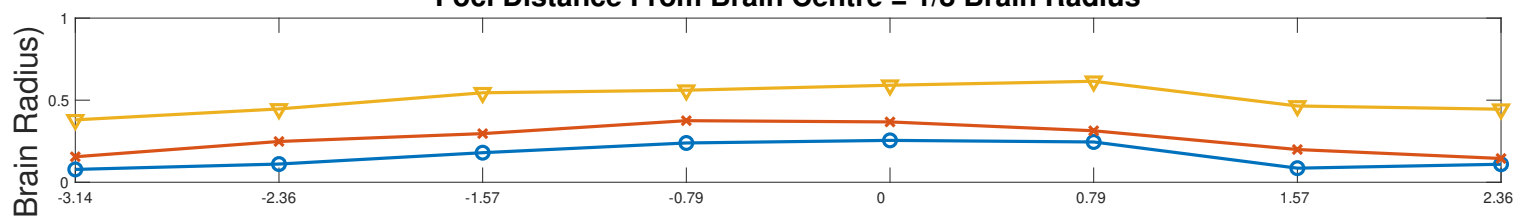

Foci Distance From Brain Centre $=1 / 4$ Brain Radius

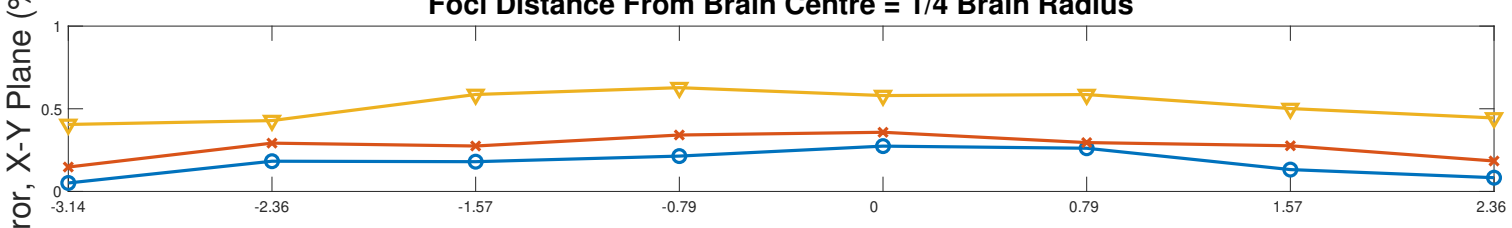

山े

Foci Distance From Brain Centre $=1 / 2$ Brain Radius

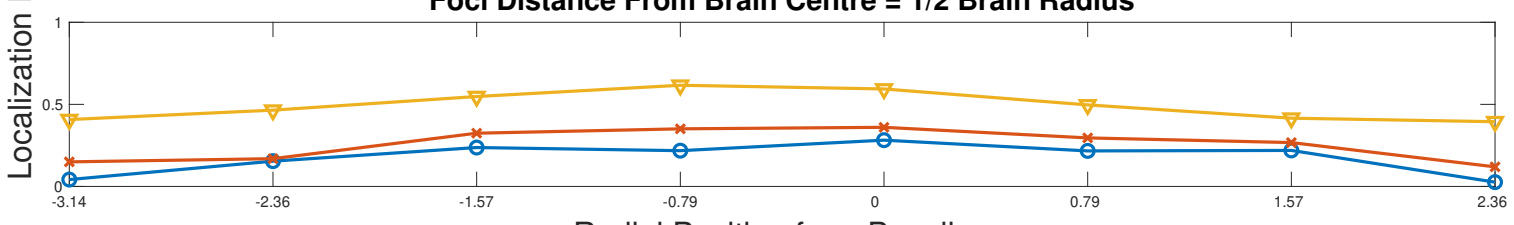

Radial Position from Baseline

Figure 4.7: Localization accuracy in the $\mathrm{x}-\mathrm{y}$ plane of reconstructed images against inclusions of known position and conductivity. Inclusion sizes were either $1 / 16,1 / 8$, or $1 / 4$ of the brain radius. Top) Inclusions of $1 \%$ conductivity decrease. Bottom) Inclusions of $1 \%$ conductivity increase.

Figure 4.9 A) and B) showed the reconstructed images from the inclusions of $10 \%$ lower and 10\% higher than background brain conductivity respectively. Despite 
Localization Accuracy for $1 \%$ Conductivity Decrease

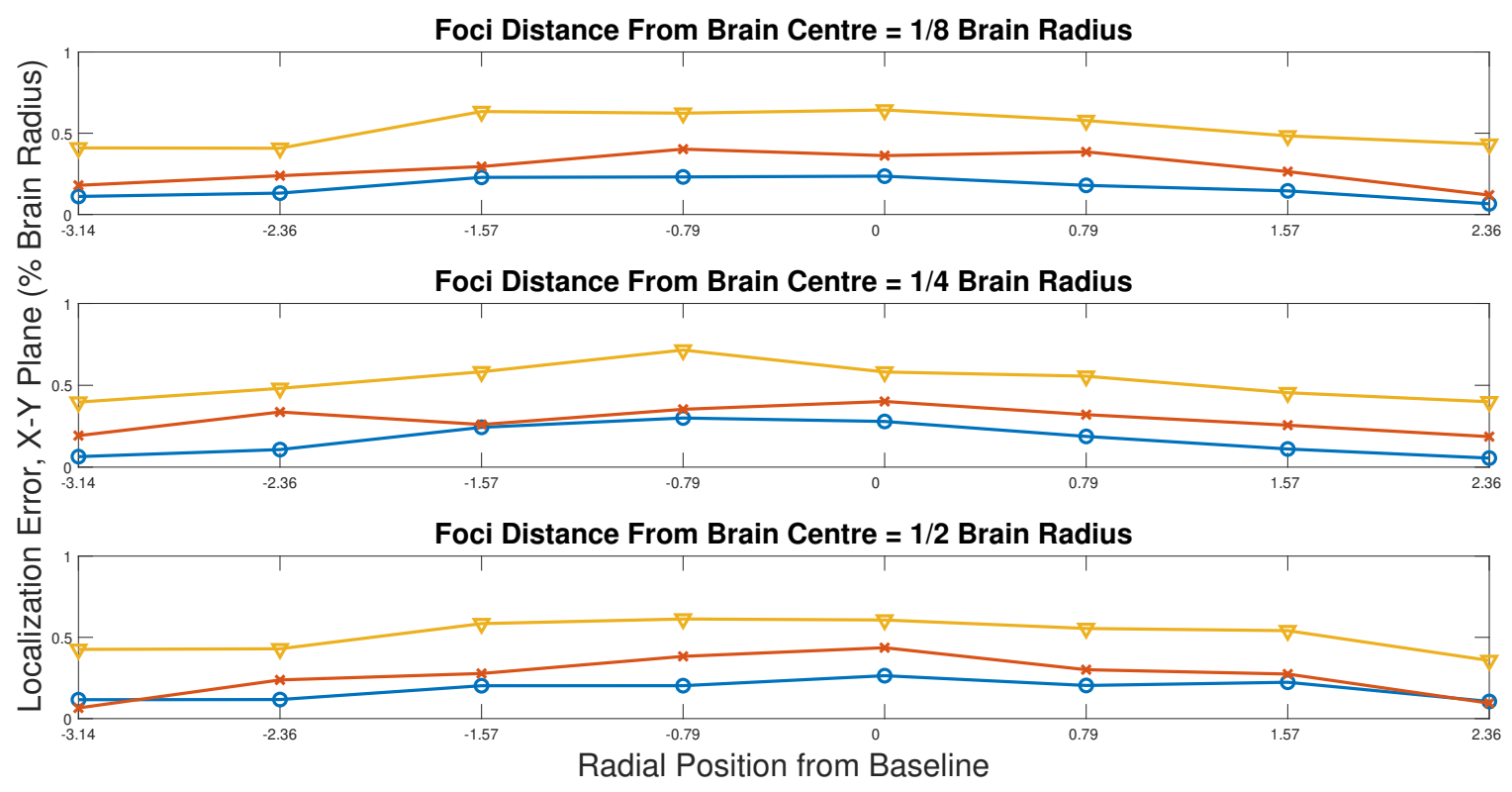

Localization Accuracy for 1\% Conductivity Increase

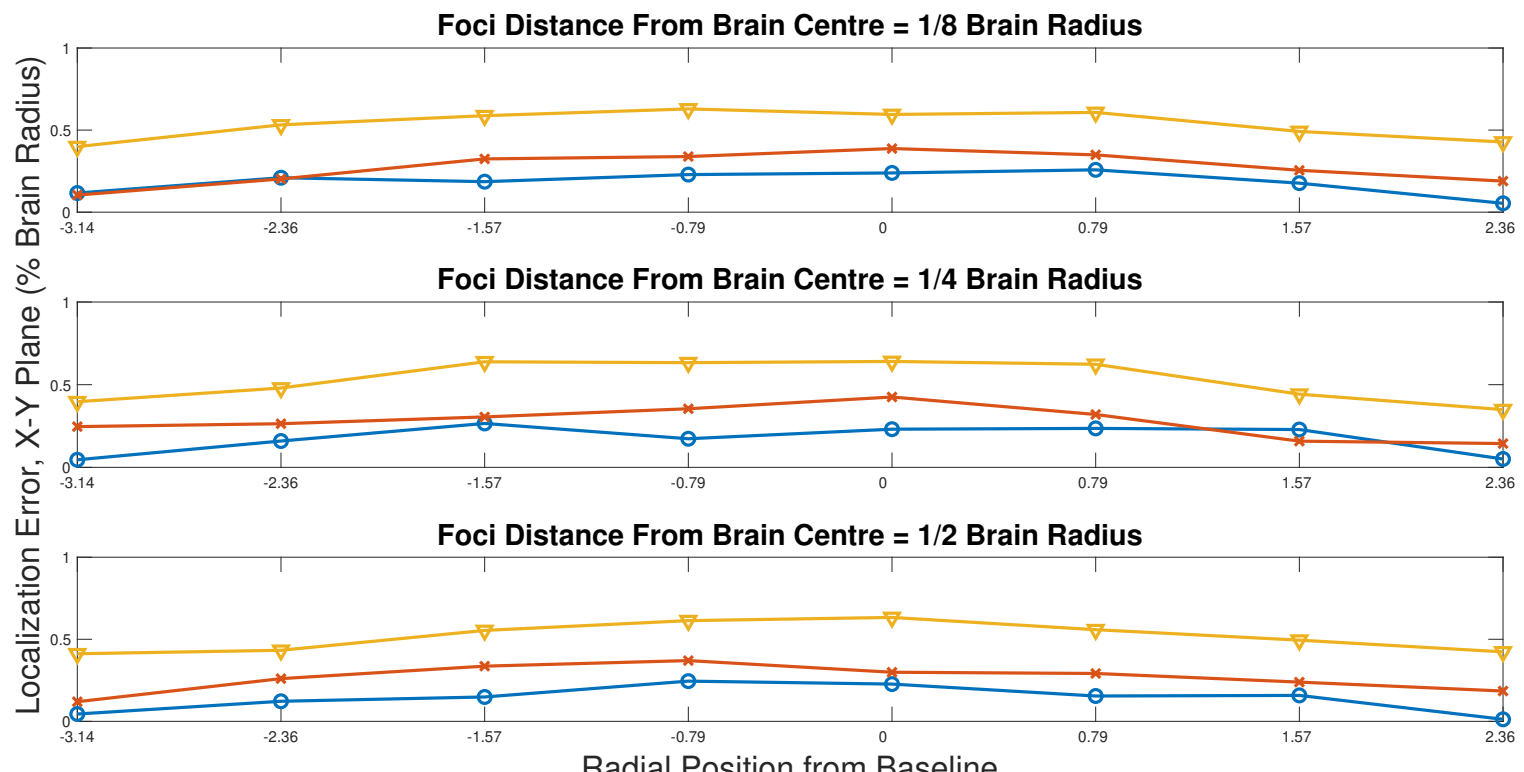

Radial Position from Baseline

Figure 4.8: Localization accuracy in the $x-y$ plane of reconstructed images against inclusions of known position and conductivity. Inclusion sizes were either $1 / 16,1 / 8$, or $1 / 4$ of the brain radius. Top) Inclusions of $10 \%$ conductivity decrease. Bottom) Inclusions of $10 \%$ conductivity increase.

the centre of gravity locations being nearly identical, low and high conductivity foci are seen near the true positions of the inclusions in A) and B) respectively. The correctness of conductivity change directions for all simulations are summarized in 
table 4.2. The $10 \%$ conductivity increases, and decreases showed the correct direction of change in $68 \%$ of images. The $1 \%$ changes showed the correct direction of change in the images for $61 \%$ and $53 \%$ of simulations for the increase and decrease, respectively.

Table 4.2: Accuracies of Conductivity Change Directions

\begin{tabular}{cccc} 
Inclusion Conductivity & Increase & Decrease & \% Correct \\
\hline $110 \%$ & $49 / 72$ & $23 / 72$ & $68 \%$ \\
\hline $101 \%$ & $44 / 72$ & $28 / 72$ & $61 \%$ \\
\hline $99 \%$ & $34 / 72$ & $38 / 72$ & $53 \%$ \\
\hline $90 \%$ & $23 / 72$ & $49 / 72$ & $68 \%$
\end{tabular}

Conductivity change directions in the true position of the inclusion from reconstructed images for each of the 4 simulations. 72 simulations for each perturbation were performed. Inclusion conductivity was expressed as a percentage of brain baseline conductivity.

\subsection{Discussion}

\subsubsection{Improvements to the electrode array}

The electrode $C_{Z}$ tests revealed that the 19-pin P75-D array had the second-lowest $C_{Z}$ on sites with hair, next to the 19-pin $\mathrm{P} 125-\mathrm{H}$ array but was the most preferred electrode due to its smaller overall size. The outer diameter of the P75-D probe head array was designed to $10 \mathrm{~mm}$, which is the same as the diameter of a $\mathrm{Ag} / \mathrm{AgCl}$ ECGtype electrode. Thus, the differences in measured $C_{Z}$ during the experiments were not influenced by differences in electrode diameter.

Given that the stratus corneum is the largest source of the resistance in biopotential electrode systems [69], the difference in $C_{Z}$ between the P75-D array and the $\mathrm{Ag} / \mathrm{AgCl}$ electrodes may have been due to the larger surface area of the latter electrode. The P75-D surface contact area, if the heads are assumed to be cylindrical, was $\pi(1.3 \mathrm{~mm} / 2)^{2} \cdot 19=25.22 \mathrm{~mm}^{2}$, compared to the $\pi(10 / 2 \mathrm{~mm})^{2}=78.54 \mathrm{~mm}^{2}$ of the $\mathrm{Ag} / \mathrm{AgCl}$ electrode. The probe spacing was set as a compromise between 


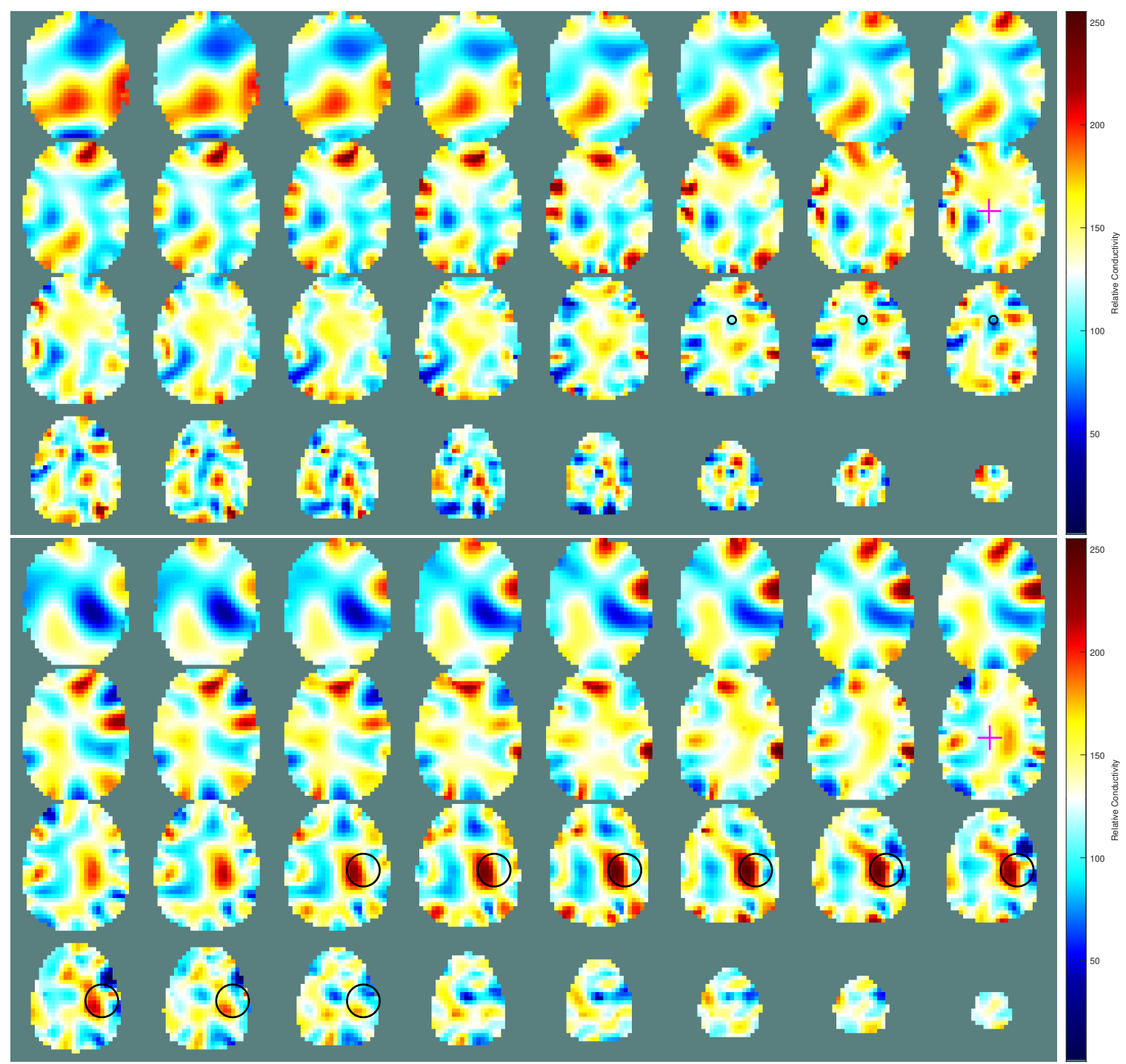

Figure 4.9: Transverse slices of the human head model from a top-down view, facing the top of the image. The size and position of the spherical inclusion for each panel is indicated by black circles. The centre of gravity for each panel is shown by the magenta crosshair. Top) Reconstructed images from a $10 \%$ lower than background conductivity inclusion of size $1 / 16$ brain radius. Bottom) Reconstructed images from a $10 \%$ higher than background conductivity inclusion of size $1 / 4$ brain radius.

minimizing overall electrode size and having spacing large enough to allow skin deformation around the dome-shaped probe heads to increase surface contact area and decrease $C_{Z}$. The spacing between probes also allowed hair to pass between pins, which prevented hair matting and allowed the probe heads to contact the scalp. The P75-D array electrode design was deemed successful because it achieved similar $C_{Z}$ 


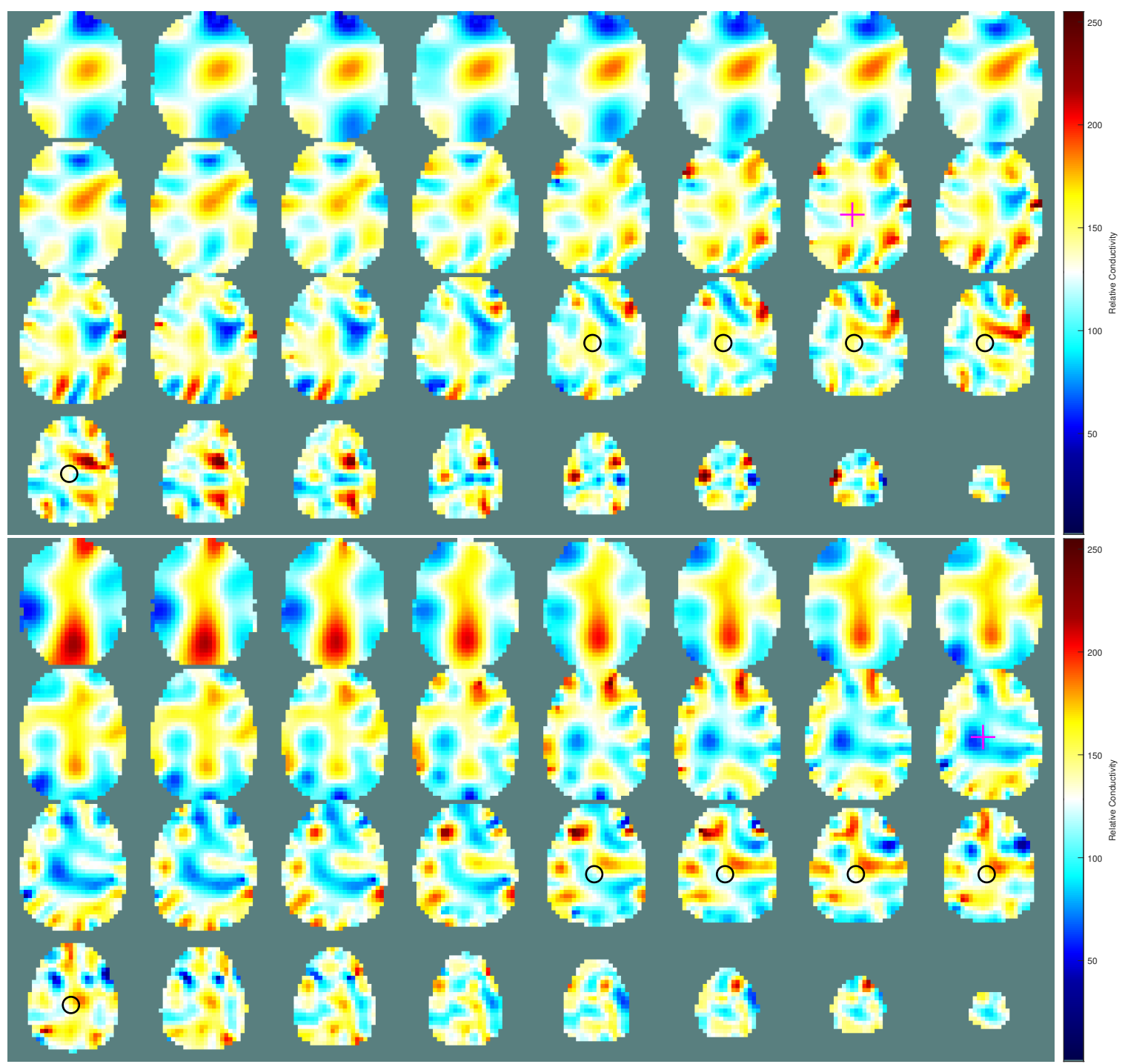

Figure 4.10: Transverse slices of the human head model from a top-down view, facing the top of the image. The size and position of the spherical inclusion for each panel is indicated by black circles. The centre of gravity for each panel is shown by the magenta crosshair. Top) Reconstructed images from a $1 \%$ lower than background conductivity inclusion of size $1 / 8$ brain radius. Bottom) Reconstructed images from a $1 \%$ higher than background conductivity inclusion of size $1 / 8$ brain radius.

to $\mathrm{Ag} / \mathrm{AgCl}$ electrodes on the forehead, while also achieving acceptable $C_{Z}$ through hair, unlike the $\mathrm{Ag} / \mathrm{AgCl}$ electrodes. Electroplating of the probe contact surfaces with Pt NPs had not yet been completed, but was expected to reduce $C_{Z}$ below those of the standard $\mathrm{Ag} / \mathrm{AgCl}$ electrodes through a dramatic increase in surface area, as shown in [66], and increase in the roughness of the contact surface [69]. Using cylin- 
drical electrode instead of dome-shaped heads may increase surface area and lower $C_{Z}$, however it has been shown that current densities at depths $>3 \mathrm{~cm}$, near the surface of the brain, when electrode spacing is $>1 \mathrm{~cm}$ is identical for cylindrical and dome-shaped electrode heads [29], and is not expected to improve performance.

The electrode housing can be improved by incorporating a screw mechanism to maintain electrode tension instead of the proposed Velcro ${ }^{\mathrm{TM}}{ }^{\mathrm{straps}}$. This design has the added benefit of allowing rotation of the probe head assembly for scalp abrasion, which was not possible with the plunger-style electrode housing shown in figure 4.2. Avery demonstrated that a single rotation of smooth-faced electrodes against the scalp achieved a great reduction in $C_{Z}$ [28]. A design similar to that of Liu et al. [66] is proposed for the next prototype (figure 4.11). Attempts at assembling prototypes of this design were made, but the difficulty of making threads by additive manufacturing resulted in poor functionality. Using a tap and die set to make this threaded joint in the electrodes is a practical solution.

\subsubsection{Simulations}

An 80 dB SNR was chosen for the simulations because previous reports have said that this effective SNR may be required for imaging scenarios of the same challenge level as functional EIT-BI, though may be successful with systems having 30-40 dB SNR [70]. The averaging of $1080 \mathrm{~dB}$ SNR measurements in the simulations was selected to reduce the computation time for simulating 100-1,000 averaged measurements for a $60 \mathrm{~dB}$ and $40 \mathrm{~dB}$ system, respectively. These large numbers of trials are realistic for the cardiac cycle ensemble method presented in chapter 3, which was intended to be employed as a long-term monitoring method, for which 1,000 trials could be collected within 15 minutes at an average heart rate of $60-70$ beats per minute.

The centre of gravity in the simulations did not change appreciably due to the 


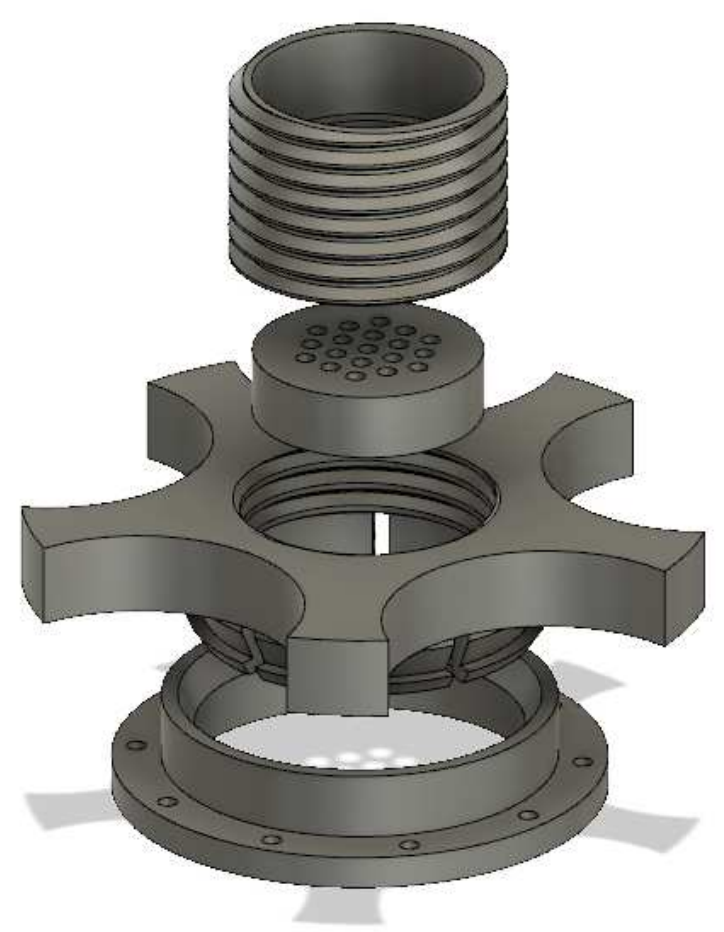

Figure 4.11: Proposed electrode design with rotating electrode housing that allows for scalp abrasion.

presence of image artifacts, thus localization error appeared to decrease the closer the true position of the inclusion was to the more-or-less fixed image centre of gravity. The results from the conductivity change direction accuracy shown in table 4.2 showed that conductivity change in the true position of the inclusion was correct $68 \%$ of the time for the $10 \%$ conductivity changes and $61 \%$ and $53 \%$ for the $1 \%$ conductivity increase and decrease respectively. The lower accuracies for the $1 \%$ perturbations indicated that improvements to the reconstruction model are required.

Improvements such as modelling the anisotropic properties of tissues, is expected to limit these artifacts and increase the model's sensitivity in future tests. The electrode stimulation and measurement patterns were not optimized for the electrode placement on this model. By re-arranging electrodes to maximize the distance between stimulating electrodes, it is expected that more current will enter the brain and increase the overall sensitivity of the model, while reducing image artifacts. 


\section{Chapter 5}

\section{Automated Electrode Quality}

\section{Assessment and Data Rejection \\ (EQADR) Algorithm}

\subsection{Introduction}

\subsubsection{Purpose}

Post-hoc EIT data analysis begins with inspection of data quality and electrode $C_{Z}$ throughout each recording, which can be a time-consuming process. This was the case for the data used in chapter 3, which had wide-ranging levels of quality that required substantial compensation for some animals. This was the motivation for creating an automated electrode quality assessment and data rejection (EQADR) algorithm for identifying and rejecting of low-quality measurements and poorly-connected electrodes. EQADR is a tool that can therefore save valuable time and increase the accessibility of EIT data analysis to users with a limited technical background. The 
EQADR algorithm was designed and implemented in MATLAB 2019a, and is compatible with the EIDORS [63] library of EIT algorithms.

\subsubsection{Defining High-Quality EIT Data}

Before describing how the EQADR algorithm works, definitions for high- and lowquality EIT data are needed. High-quality EIT data collected from biological systems typically has the majority of its spectral power concentrated below $5 \mathrm{~Hz}$. EIT data may contain large amplitude, low frequency signals, but the mean absolute value of the data's derivative should be small. These characteristics are true of data collected using the SenTec Pioneer Set and may differ for other devices. Low-quality EIT data is characterized by measurements whose voltages exceed the measuring capacity of the EIT hardware. When the real (voltages) and complex (phase angle) components of the data are plotted together, these "clipped" measurements fall within a ring around the edges of the real-complex plane, normally caused by sharp increases in electrode $C_{Z}$. Movement artifacts, spurious electrode disconnections, and/or other faulty hardware connections also cause large increases in electrode $C_{Z}$ that produce anomalously large voltage readings.

\subsubsection{Methods of Detecting Faulty Electrodes and "Bad" Measurements}

With the wide range of electrodes used in EIT systems, a mean or median $C_{Z}$ threshold is not sufficient for identifying faulty electrodes. Electrodes with low $C_{Z}$ that have several disconnections within a long time series will have low average $C_{Z}$ that can be missed by thresholds. Other electrodes that have higher $C_{Z}$ than standard $\mathrm{Ag} / \mathrm{AgCl}$ electrodes may be rejected by thresholds in the upper ranges of their normal $C_{Z}$ 
variability. More importantly, the reciprocity of EIT stimulation and measurement patterns means that each electrode participates in two stimulating and measurement pairs. If one of these three electrodes are faulty, all three may exceed threshold $C_{Z}$ and become discarded by a naive approach.

\section{Zeroing bad measurements}

Measurements from faulty electrodes must be discarded to improve image quality. The EIDORS software package [63] used for EIT data analysis has functions for zeroing measurements from user-specified electrodes, however this approach has been shown to introduce distortion to images [71].

Recall from section 2.2.2 that the Jacobian matrix $J$ linearizes the forward model at a given reference conductivity frame. The values in $J$ represent the voltage change from reference values, for each of the $m$ measurements, for a given conductivity perturbation $\sigma_{x}$, at each of the $n$ image elements. Therefore, a change in any one measurement affects the conductivity values at every image element. Zeroed measurements are treated as true 0 -valued measurements in image reconstruction, thus the images reflect a system in which no voltage change occurred at these locations, which is a reasonably safe approach, but does not reflect the system's ground truth, and produces image artifacts.

\section{Updating the Measurement Cross-correlation Matrix}

Instead of zeroing noisy measurements, Adler proposed an approach that removed their contribution to the reconstruction matrix, by updating the measurement cross-correlation matrix $X$ by the ratio of expected to actual measurement crosscorrelations, which produced undistorted images [71].

A change from reference conductivity $\Delta \sigma$ is related to the change from reference 
measurements $\Delta d$ by:

$$
\Delta d=J \Delta \sigma=J R \Delta d
$$

Where $J$ is the Jacobian and $R$ is the reconstruction matrix, formulated as:

$$
R=P J^{T}\left(J P J^{T}+X\right)^{-1}
$$

Where $P$ is a regularization matrix that stabilizes image element values in the presence of changing measurements by incorporating a priori knowledge that an element's sensitivity to changes in measurements is inversely proportional to its distance from the body's centre [20].

$X$ is computed as follows: the matrix $Y$ is computed, which represents the noise amplitude of each measurement $m$ at each image element $n$, calculated as the ratio of voltages after the conductivity perturbations $\sigma_{x}$, with respect to the reference frame voltages, obtained during the calculation of $J$. The average measurement noise amplitude $\sigma_{n}$ is then determined as the mean absolute value of these voltage ratios. Given the independence of measurements, the expected measurement crosscorrelation matrix $\Sigma_{n}=I$. The regularized measurement cross-correlation matrix $X$ is then:

$$
X=\left(Y Y^{T}+\sigma_{n}^{2} \Sigma_{n}\right)^{-1}
$$

When a new measurement cross correlation matrix $X_{1}$ is calculated from real data, a noisy measurement $i$ will be uncorrelated with clean measurements, thus:

$$
\widetilde{Y}_{(i, i)} \widetilde{Y}_{1(i, i)}^{T} \approx 0,\left(Y Y_{i, i}^{T}\right)^{-1} \approx \infty
$$

and

$$
\sigma_{n}=\widetilde{X}_{(i, i)} / X_{(i, i)} \approx \infty
$$


The contribution of noisy measurement $i$ to reconstructions can then be zeroed in $X$. Mamatjan [72] extended this work by formulating the update of $X$ in a fast and efficient way - by a series of rank-1 updates to $X$ :

$$
\widetilde{X} \rightarrow X-X_{(i)}^{T} X_{(i)}\left(X_{(i, i)}\right)^{-1}
$$

Therefore, this operation removes contribution of measurement $i$ to $X$. This method carries out this operation before image reconstruction so that the effect of the noisy measurement is removed from the reconstruction matrix altogether, while the contribution of the remaining measurements is updated accordingly. Clean data $\tilde{d}$ can then be interpolated from noise-contaminated $d$ by:

$$
\tilde{d}=J P J^{T} \tilde{X} d
$$

\subsection{Methods}

\subsubsection{Identification of Erroneous Measurements}

While the approach for eliminating noisy measurements from reconstructions had been developed elsewhere, a method for detecting noisy data was created in this work and paired with the work Adler and Mamatjan to create EQADR.

The data and inverse model are first fed into a function that assigns a score to each electrode and measurement pair, based on the proportion of low-quality measurements identified to the total number of measurements taken with that electrode or measurement pair. The user may decide whether all measurements from a specifiable maximum number of faulty electrode(s) should be discarded, or if data from individual low-quality measurement pairs should be discarded. The latter case is use- 
ful for scenarios in which there is a large $C_{Z}$ discrepancy between an electrode and its two partners, such as if a high impedance path exists between one pair and not the other. The former case generally discards more measurements than the latter but is useful for truly faulty electrodes.

Measurement or electrode rejection is guided by a user-defined threshold ranging from $0-1$. The threshold represents the maximum proportion of noisy samples a measurement or electrode may have before they are rejected. The maximum number of measurements or electrodes that can be rejected is also determined by the user, with rejection starting with the worst measurement or electrode, then proceeding until no more data groupings are higher than threshold, or until the maximum number of rejections occurs. The threshold parameter allows the user to tune the true positive and false positive rates for data rejection for their dataset and analysis.

Noisy measurements are defined as those that are clipped, and/or those with a negative voltage reading (figure 5.1). Clipped measurements fall on the boundaries of the real-complex plane and form a circular shape centered at the origin. Thus, a circle centered at the origin with a radius slightly larger than the distance from the clipped measurements to the origin would encompass all data points in the plot shown in figure 5.1. A circle with a radius slightly smaller than the distance between the origin and clipped measurements would contain nearly all the clean data, but not the clipped measurements. This is the principle behind how EQADR detects clipping.

In the EQADR algorithm, the largest distance from the data to the origin is first determined. A circle with radius 5\% larger than this distance would contain all data points. To find the boundary between clean and clipped data, an expanding circle is drawn, and the number of data points that fall within that circle are counted. Given that clean EIT data clusters along a line angled from $0-45^{\circ}$ from the real (x) axis, the number of new data points falling within the expanding circle is a logarithmic or 
linear function, up until a small "blip" in the function appears when the clipped data is encompassed by the circle. All data points outside of the circle at the iteration before this inclusion spike occurs are labelled as noise, and all data within the circle are labelled as clean. Next, any remaining data with a negative real component is also labelled as noise.

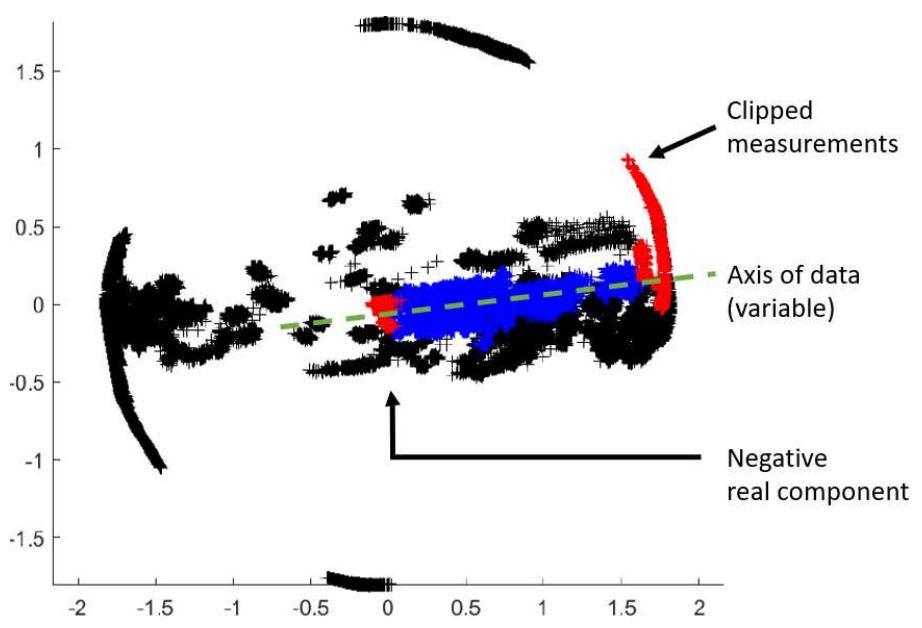

Figure 5.1: Plot showing the real and complex components of sample impedance data. All data was plotted in black. Data selected to influence the reconstruction matrix were then plotted in blue. Clipped measurements and those with negative real components are highlighted in red. The green line shows the line or "axis" upon which EIT data normally falls.

The reciprocity of EIT measurement and stimulation patterns implies that a single faulty electrode will cause the other two electrodes with which it pairs (its "partners") to appear faulty also, even when they are not. This can be seen in figure 5.2, where 3 electrodes are seen above threshold. A skip-4 pattern was used for these measurements, meaning electrode 1 pairs with electrodes 6 and 28. The higher score for electrode 1 implies that it is noisy, and the lower scores of electrodes 6 and 28 imply that they are not noisy. If they were, their scores would be expected to be as high as electrode 1's, and the other partners of electrodes 6 and 28 would have higher scores because of it. The contribution of electrode reciprocity to the scores is resolved by a method shown in this pseudocode: 
for each of the $\mathbf{i}$ electrodes:

find the electrodes $\mathbf{p}_{\mathbf{1}}$ and $\mathbf{p}_{\mathbf{2}}$ that pair with electrode $\mathbf{i}$.

for each of the $\mathbf{p}_{\mathbf{i}}$ partners of $\mathbf{i}$ :

find the partner $\mathbf{p}_{\mathbf{i p}}$ of $\mathbf{p}_{\mathbf{i}}$ that is not $\mathbf{i}$.

If the electrode score of $\mathbf{i}$ is greater than or equal to the combined scores of $\mathbf{p}_{\mathbf{i}}$ and $\mathbf{p}_{\mathbf{i p}}$, $\mathbf{p}_{\mathbf{i}}$ is not noisy. Reflect this by setting the score of $\mathbf{p}_{\mathbf{i}}$ to that of $\mathbf{p}_{\mathbf{i p}}$.

Figure 5.2 shows that when score resolution was applied to the example data, electrode 1 remained above threshold but all other electrodes fell below threshold. The impact of score resolution on retained and rejected data, as well as validation that faulty electrodes were not mislabeled, is presented in the next section. After score resolution, the impacts of faulty measurements identified by EQADR on reconstructions are then removed using the method described in section 5.1.3. 

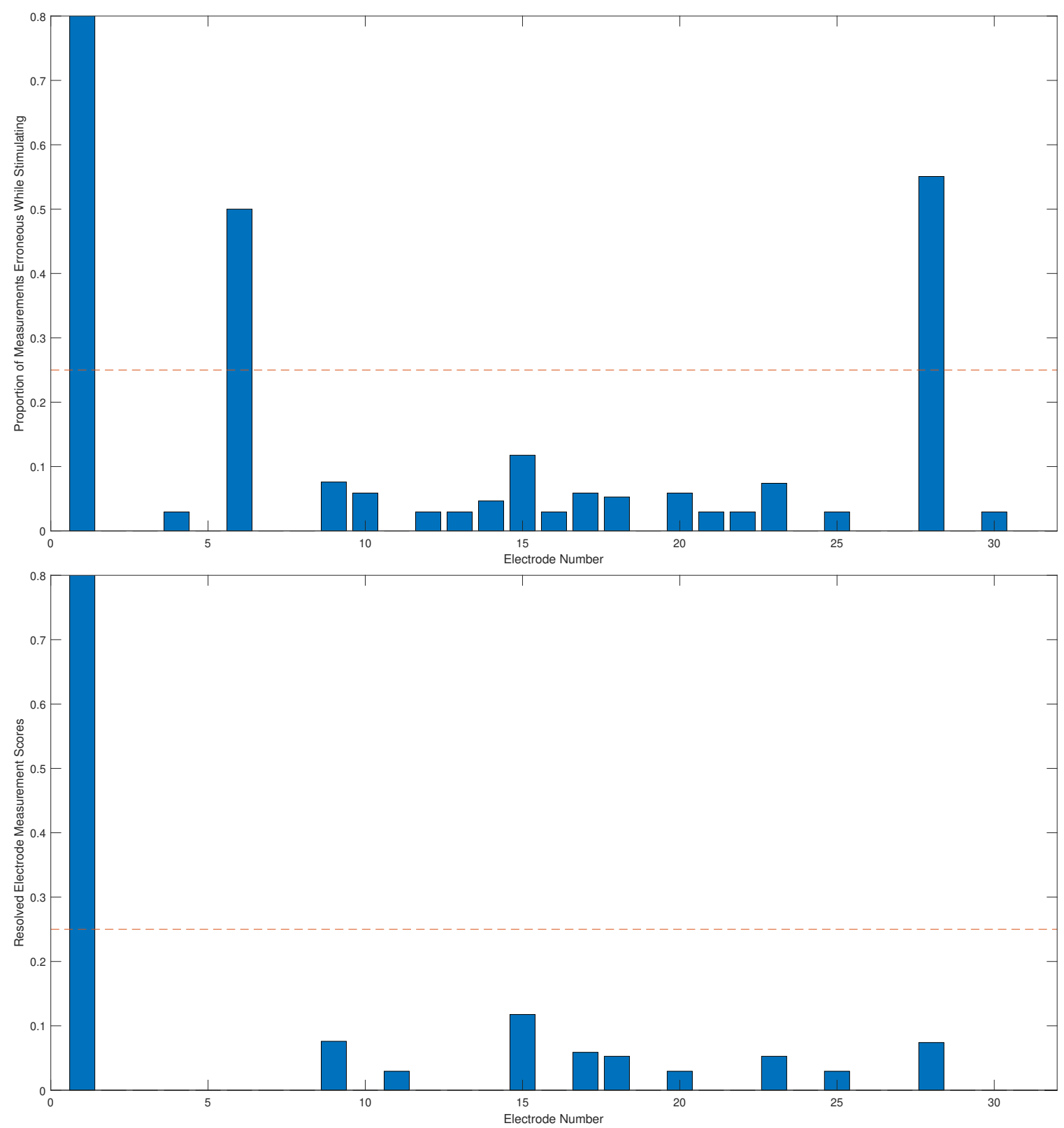

Figure 5.2: Electrode scores when not resolving (top) and resolving (bottom) electrode reciprocity in electrode scores. The threshold for the maximum score is shown by the dashed red line.

\subsection{Results \& Discussion}

The effect of the electrode score resolution function on data retention is shown in figure 5.3, using EIT stroke data with a skip-4 pattern from the baseline condition 
of animal B presented in chapter 3. All data points were first plotted in black. The stimulation/measurement pattern specified that data from the two adjacent measurement pairs on either side of the stimulating pair would be rejected. All retained data were either plotted in blue, red, or magenta. Red markers represent data that would be excluded by the measurement rejection scheme, while both the red and magenta markers would be excluded by the electrode rejection scheme. Data that would be retained by EQADR are plotted in blue.

The top panel of figure 5.3 shows that without score resolution, electrodes 1,6 , and 28, which formed stimulation / measurement pairs in the skip-4 pattern, were all identified as noisy. The rejection of these three electrodes resulted in 130 rejected measurements. With score resolution, shown in the bottom panel of figure 5.3, only electrode 1 was identified as being noisy, and 68 measurements were rejected, a difference of 62 . The retained data from electrodes 6 and 28 clustered with the clean data, seen by the replacement of magenta points with blue points between the first plot in the top and bottom panels of figure 5.3, despite the high median $C_{Z}$ of electrode 28 . The magenta points falling off the clean EIT data axis can be seen to have belonged exclusively to electrode 1.

Figure 5.4 shows data collected from animal B in chapter 3 at baseline before saline injection. The brain is in the middle-top of these images (light circular region in the second and third images). With no compensation, shown in the first image, the right side of the brain in the image is below baseline conductivity levels, and there are high-amplitude noise artifacts throughout the image. With compensation but without score resolution (figure 5.4 second image), the overall noise level is reduced, including in the brain region. With score resolution (third image), the boundary lines between conductivity changes above and below baseline become sharper due to the increased overall sensitivity of the system with higher data retention. 

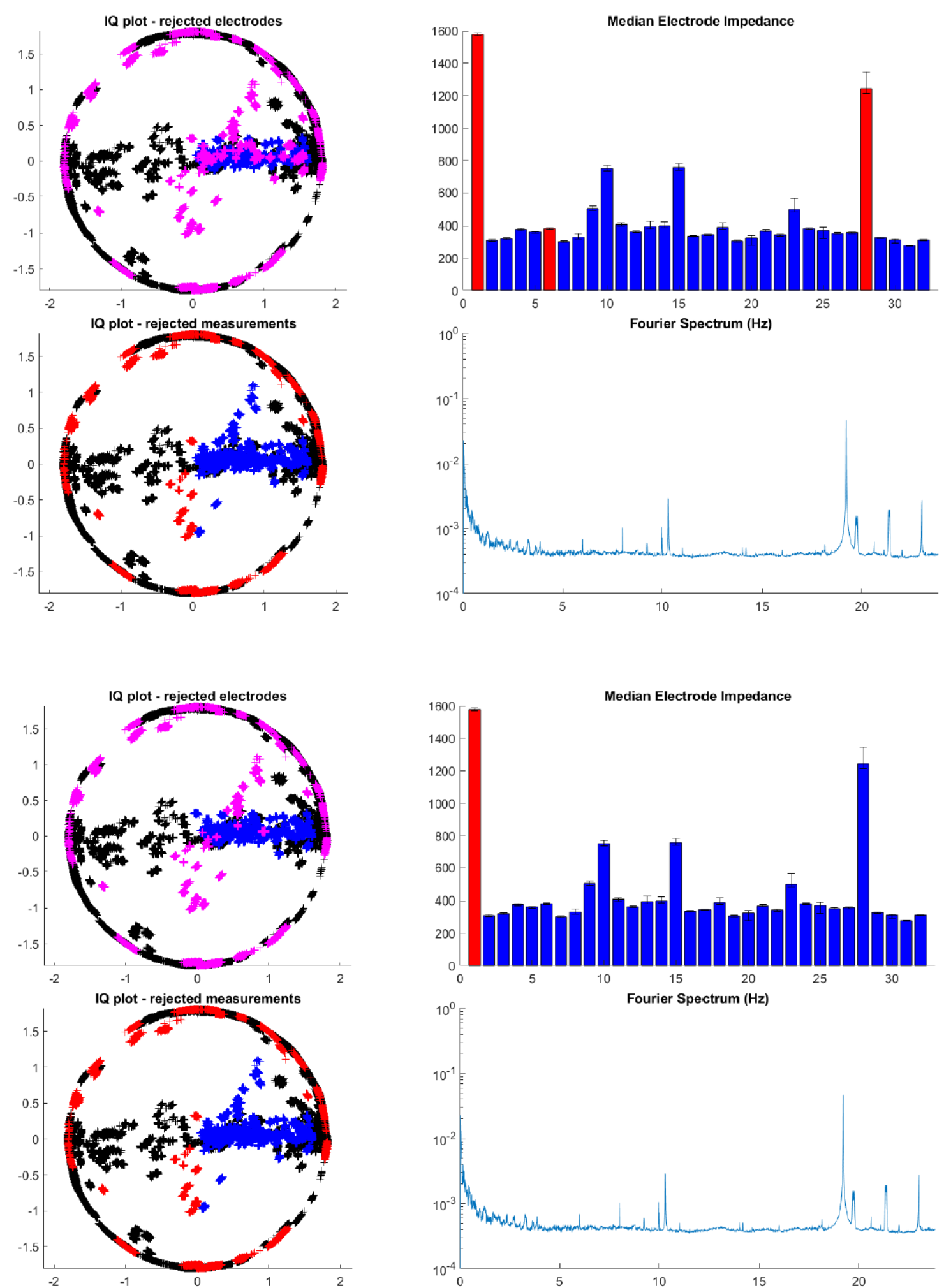

Figure 5.3: Difference in data retention when not resolving (top) and resolving (bottom) electrode reciprocity from electrode scores. All data was plotted in black. Data selected to influence the reconstruction matrix were then plotted in blue. Data that would be rejected by full exclusion of the detected faulty electrodes were then plotted in magenta, followed by the data belonging to individually rejected measurements in red. Without resolving for reciprocity, two additional electrodes were selected for rejection. The difference in the number of magenta points between cases shows that resolving scores retains quality data that would otherwise be discarded. 
The final image in figure 5.4 was formed by subtracting the unresolved image from the resolved image. The difference image showed that not rejecting electrodes 6 and 29 affected pixel values throughout the image, emphasizing the importance of data retention.

The results of this example demonstrated how EQADR improves image quality by retaining data that would otherwise have been discarded by thresholding methods or unresolved electrode scores. This fully automated tool was intended to be a component of a larger automated pipeline that processes raw EIT data into images. The goal of EQADR was to increase the accessibility of EIT to new researchers and to medical professionals, who may have minimal technical EIT experience, but desire robust and reliable EIT images for downstream use.

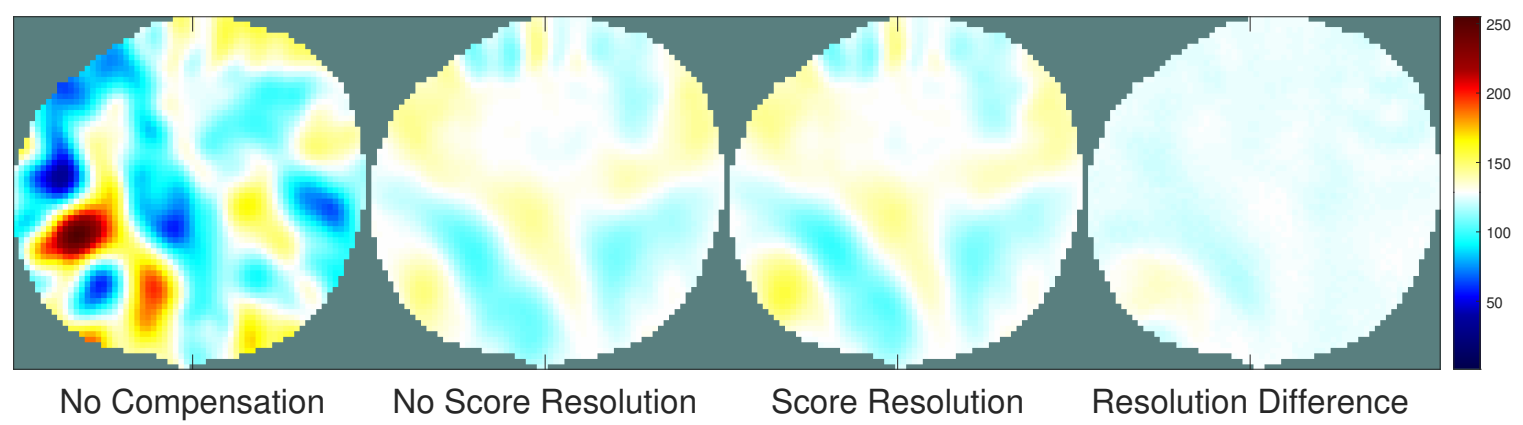

Figure 5.4: Reconstructed images from swine EIT stroke data without compensation (first image), with compensation but without score resolution (second image), with compensation and score resolution (third image) and the difference between no score resolution and score resolution (fourth image).

In the future, EQADR can be improved by locating the EIT data axis and marking as noisy data points that are outside a user-defined distance from this line. In some cases, clusters of data such as the top magenta cluster in the upper panel of figure 5.3 are missed by EQADR, because they neither have a negative real component nor are clipped. A distance threshold would therefore increase the robustness of EQADR in these edge cases. 


\section{Chapter 6}

\section{Discussion \& Future Work}

\subsection{Summary}

The goals of this work were to develop EIT methods and hardware for detecting $\mathrm{CBV}$ changes that occur in ischemic stroke, and to explore the feasibility of imaging functional brain activity with EIT. Ischemia was the target of the two perfusionbased methods presented in chapter 3 because hemorrhage, but not ischemia, had previously been imaged with EIT. The progress made towards preparing these EIT methods for human studies was presented in chapter 4, where the realistic human head reconstruction model, novel polarizable EIT electrodes, and electrode headset design were described, and the results of simulated stroke and neural activity were presented. An REB-approved experimental protocol was created for this functional imaging study and is presented in appendix B. A semi-automated software tool was built to deliver this protocol, coordinate data synchronization, and to record the timing of stimulus presentation and user interactions with the software. EQADR, which was built as a component of an automated EIT reconstruction pipeline needed for clinical adoption of brain EIT, was presented in chapter 5. Finally, the results 
from an experiment examining the effects of weighted restraint on the distribution of ventilation is presented in appendix A.

\subsection{Discussion}

\subsubsection{EIT Stroke Methods}

The results from section 3 provided the first demonstration that EIT can detect cerebral ischemia by monitoring relative CBV changes over averaged cardiac cycles. The perfusion-based saline contrast imaging was less effective. Ischemia was detected in the correct location for $4 / 5$ animals. This study provided sufficient confidence in this technique to warrant study in humans.

\subsubsection{EIT Electrode Design}

A rotatable, retractable electrode housing that incorporated a 19-pin array of P75-D spring contact probes was designed to permit easy adjustment of electrode tension against the scalp, easy clean-up, abrasion of the scalp via rotation of the probe head assembly, easy replacement of probe heads, and mounting to a headpiece to form the headset montage. This electrode design was tested alongside other experimental electrodes for their $C_{Z}$ on the scalp in the presence of thick hair, on the forehead, and on the forearm. The P75-D array performed the best overall, with a $C_{Z}$ of $750 \Omega$ on opposite sides of the head through thick hair, and performed similarly to the $\mathrm{Ag} / \mathrm{AgCl}$ electrodes on the forehead and forearm, achieving $C_{Z}$ of $475 \Omega$ and $350 \Omega$ when used with ultrasound gel, versus $400 \Omega$ and $350 \Omega$ of the $\mathrm{Ag} / \mathrm{AgCl}$ electrodes on the two sites respectively. 


\subsubsection{Realistic Human Head Reconstruction Model and Sim- ulations}

A realistic human head reconstruction model was created, having 3,688,836 elements with an average Delaunay triangulation quality of 0.72 . An algorithm for locating the electrode centre locations on the surface of the model for electrode labelling and mesh refinement was created and employed. An advantage of this algorithm is that it allowed customization of the number and locations of electrodes on the model, enabling the easy production of reconstruction models for future headset prototypes.

Simulations of $10 \%$ conductivity increases and decreases typical of hemorrhage and ischemia, using an SNR of $80 \mathrm{~dB}$, revealed that the localization error in the $\mathrm{x}-\mathrm{y}$ plane was adequate, but the $\mathrm{z}$ error was large due to artifacts below the electrode imaging field. Simulations of $1 \%$ conductivity increases and decreases showed more spurious artifacts, though mildly interpretable images.

\subsubsection{N-Back Graphical User Interface (GUI) Software}

A GUI was created in MATLAB 2019a for the automated delivery of an n-back working memory task from user-specified parameters of " $\mathrm{n}$ " value, number of stimuli, and number of correct responses. A randomized n-back deck is built from these parameters each time the task is started. This software increased the ease of protocol delivery and post hoc analysis by automatically acquiring, labeling, and storing synchronized EIT and ECG data, along with the relative timing of stimulus presentation to data acquisition, and user interaction with the software. 


\subsubsection{Weighted Restraint}

This study demonstrated that loss of lung reserve volume was a common occurrence of weighted restraint, and volume loss increased with duration of exposure to the applied static inspiratory restive load on the back of subjects.

\subsection{Future Work}

The results of the two perfusion-based EIT-BI techniques may be improved with higher quality data and a higher number of averaged frames for the ensemble method.

While circumstances did not allow for the completion of the electrode headset, several electrode prototypes were built and tested. The $C_{Z}$ of the 19-pin P75-D array can be improved by coating the probe contact surfaces with Pt-NP as in [66]. The electrodes can be further improved by manufacturing the design shown in section 4.3.1 with rotatable probe housing, which would permit scalp abrasion and a further decrease in $C_{Z}$ to improve data quality.

The overall mesh quality could be improved by eliminating the diploe segmentation, which had small-volume, distributed voxel clusters, which may have led to irregularly shaped tetrahedra that reduced overall mesh quality. The shape of the white and grey matter segmentations could be smoothed to improve the generalizability of this model, as different individuals will have arrangements of these tissue, and the shape within the model should have reflected the average shape. The mesh incorporated the conductivity values of each tissue type; however, these were represented as isotropic conductivities. Some tissues such as the skull and white matter are highly anisotropic, and modelling them as such has been shown to reduce the occurrence of spurious artifacts in reconstructions compared to an isotropic model, when data were simulated using an anisotropic forward model [73]. The stimulation and 
measurement patterns, as well as the electrode numbering were not optimized. The reconstructions can be improved by optimizing the electrode numbering and stimulation/measurement patterns to maximize the distance between stimulating electrodes to increase current density within the head.

The COVID-19 pandemic prevented data collection for the human EIT-BI study, though a plan is in place to begin data collection once it is safe to do so. The REBapproved EIT-BI protocol must be first be appended to include ECG collection and re-submitted with this change. The appearance of the n-back protocol software can be improved, though is not necessary, as the software is functional. Once the electrode headset has been assembled, the software will be tested in a dry-run experiment to assess user experience and fix any software bugs that may arise.

The weighted restraint study was submitted to Physiological Measurement but was rejected for its small study size. A larger population of 30 subjects, 10 for each posture, is required to improve the statistical power of the findings. Data from 14 additional subjects has been collected and is planned to be added to this study.

\subsection{Conclusion}

In summary, this thesis has provided evidence for an EIT-BI technique capable of imaging ischemia in a pig model of stroke. This technique is expected to yield equal or better capability for detecting of cerebral perfusion deficits in humans, in whom the distance between electrodes and skull is smaller, the skull thinner, and the brains larger, than in pigs. Great progress was made in this work towards readying these techniques for human studies. With a modest time investment, and using the path set out in this work, the electrode headset can be completed and these techniques will be available for human experiments. 


\section{Appendix A}

\section{Using Electrical Impedance}

\section{Tomography in an Experimental}

\section{Model of Weighted Restraint}

This appendix presents an article that was submitted to Physiological Measurement, but was rejected on the basis that the number of participants was too low. New data was collected, and the manuscript has been re-written. This manuscript is included in this thesis to ensure that in case of a second rejection, the results, which have important implications on restraint asphyxia and police tactics, are published, despite the topic being unrelated to brain imaging.

The protocol was designed by Symon Stowe, who also produced the methods figure

for this article. Data was collected by the author, Brianna Raven, Kira Burton, and Mali Mapani. The author performed the analysis and writing of the manuscript.

Title:

Using Electrical Impedance Tomography in an Experimental Model of Weighted Restraint 
Authors:

Mark Campbell, Symon Stowe, Brianna Raven, Kira Burton, Malitela Mapani, Jeff W. Dawson, Andy Adler

\section{A.1 Abstract}

Restraint asphyxia has been proposed as a mechanism for some arrest-related deaths that occur during or shortly after a suspect is taken into custody. While this theory is controversial, our analysis of the literature indicates the combined effects of prone positioning, weight applied to the back, recovery after simulated pursuit, and restraint position have not been tested together; however, subsets of these variables lead to restrictive, but non life-threatening, respiratory changes. We hypothesize that a more complete protocol with more sensitive instrumentation can improve our understanding of breathing physiology in this setting. We designed an electrical impedance tomography (EIT)-based protocol for monitoring respiratory adaptations during weighted restraint and measure the 3D distribution of ventilation within the thorax. Here, we present the results from a pilot study on 17 subjects. We found a pattern of fatigue that occurs during restrained recovery from exercise, in which a subject's ability to maintain their lung reserve volume was compromised despite normal recovery of minute ventilation. Moreover, this "reserve volume collapse" was abolished by the removal of the weighted load.

\section{A.2 Introduction}

The term "weighted restraint" describes the application of an external load to a subject's back while they are restrained or are being restrained. Weighted restraint 
is frequently used in law enforcement, where officers apply their body weight to a subject to control their movements during apprehension. Though rare, arrest-related deaths (ARD) do occur, and in some cases the cause of death is inexplicable or unknown [74]. Restraint asphyxia, of which weighted restraint is a component, has been investigated as a putative mechanism for some of these ARDs $[75,76,77,78$, 79, 80]. Four prominent factors - physical exertion, prone positioning, restraint, and body compression - appear in the statistics of ARDs [81] and have been tested in the literature, but have not been tested together in a single study. To address this gap in the literature, we have developed a methodology for measuring the combined impacts of these parameters on ventilation using electrical impedance tomography (EIT), which extends our previous work on the topic [82].

EIT is an imaging modality that produces images of internal body structures by reconstructing changes in they body's internal conductivity distribution non-invasively. The large difference in conductivity between body tissues and air makes EIT ideal for respiratory monitoring. Surface electrodes are placed on the body in a pre-determined geometry, then small electrical currents are iteratively passed between unique pairs of electrodes, while measuring resulting voltages from other non-injecting electrodes. With electrodes in a three-dimensional configuration, EIT provides information on both large scale parameters such as respiratory rate, tidal volume, and changes in function residual capacity, but also smaller scale changes such as 3D changes in lung filling and ventilation distribution [83].

The use of EIT monitoring in weighted restraint makes two important contributions to this application. First, using only body-surface electrodes, EIT has a minimal encumbrance to the research participants' breathing, since no mask or other device is required to be worn on the face. An EIT-instrumented breathing protocol can thus be more comfortable and potentially more realistic. Next, EIT produces an image 
or 3D volume of the distribution of air within the thorax. Using these data, functional imaging parameters can be calculated which are more sensitive than global parameters from other methods to changes in lung physiology [84].

Restraint asphyxia itself has been a controversial topic. The "restriction of thoracic respiratory movements" imparted by a restraint position, together with exertion from pursuit, was initially suggested as a mechanism for inexplicable ARDs [75]. Subsequent studies have found non life-threatening decreases in forced expiratory volume and forced vital capacity after exercise during prone restraint compared to a seated position [76]. Others have observed independent significant reductions in maximum voluntary ventilation in weighted unrestrained prone subjects and in unweighted restrained prone subjects compared to the seated position [78]. These results suggested that physical exertion, prone positioning, restraint, and body compression impair normal respiration, but the accumulation of these effects have not yet been tested and may play a role in ARDs. The EIT-based protocol was designed for this study to improve understanding of lung mechanics during weighted restraint, monitor the cumulative impairments with high sensitivity, while ensuring safety for the participants.

In this protocol we tested 2 different restraint postures against a control posture to investigate whether some restraint postures are better tolerated than others. We analyzed minute ventilation (MV), change in functional residual capacity $(\Delta \mathrm{FRC})$, and how inflation of the lungs within the chest wall was impacted by restraint position while under load via center of ventilation $(\mathrm{CoV})$. 


\section{A.3 Methods}

This study took place at Carleton University and was approved by the Carleton University Research Ethics Board, protocol \#108481. Nineteen healthy male subjects under the age of 50 (mean: 25.76, range: 19 - 44) were recruited for this study. Subjects were excluded from this study based on any of the following criteria: a history of anxiety or panic disorders, having not exercised for more than 30 minutes in the last 30 days, being older than 50 years of age, having asthma, answering "yes" to having been diagnosed with a heart or lung condition, feeling unwell, or having a history of back issues. Male subjects were selected for this study because males comprised $95.4 \%$ of ARDs [74].

The age, weight, and height of each subject was first recorded. The subject's maximum heart rate (MHR) was estimated using the 220 - age formula, minus a 10 beat per minute safety factor. A total of 32 ECG-type electrodes were placed in 2 parallel planes of 16 electrodes using anatomical landmarks to allow 3D imaging of the subjects' thorax. The bottom electrode plane was set $4 \mathrm{~cm}$ below the xiphoid process and the top electrode plane was set $6 \mathrm{~cm}$ above the bottom plane. The chest circumference along each electrode plane was then divided into 16 even spaces and marked with a grease pencil. The stratus corneum was then abraded with Nuprep gel applied with a cotton swab, until the grease pencil marks had been removed. Electrodes were then applied over the prepared skin and connected to the SenTec EIT Pioneer Set in a square pattern, ensuring vertical alignment of electrodes across planes. EIT measurements were collected using currents of $3 \mathrm{~mA}$ peak-to-peak at $195 \mathrm{kHz}$ in a skip-4 injection and measurement pattern.

The protocol, summarized in figure A.1, consisted of 5 experimental phases: standing upright for 2 minutes (U), prone with arms at the side for 2 minutes (R), prone 
with arms at the side with weight for 5 minutes $\left(\mathrm{W}_{1}-\mathrm{W}_{5}\right)$, prone in a control posture or one of two restraint postures with weight after exercise for 5 minutes $\left(\mathrm{X}_{1}-\mathrm{X}_{5}\right)$, and prone in the same posture without weight for 2 minutes $(\mathrm{P})$. Throughout each recording, subjects' state of consciousness was monitored by the research team using the Glasgow Coma Scale (GCS) [85] once every 2 minutes. Recordings with subjects in the prone position took place on a hard foam exercise mat laid over a wooden table, covered with a clean sheet. To breathe comfortably while prone, subjects were given the choice of resting their foreheads on a horseshoe-shaped pillow or turning their heads to the side. In phases $\mathrm{W}$ and $\mathrm{X}, 35 \%$ of the subjects' bodyweight \pm $1 \mathrm{~kg}$ in bagged sand was placed on the subject's back, centered over the scapulae. Between phases $\mathrm{W}$ and $\mathrm{X}$, subjects were transferred to an exercise bike and pedalled for a maximum of 10 minutes, or until they had maintained between $70 \%$ and $85 \%$ of their MHR for 3 consecutive minutes. Exercise was terminated if HR exceeded 85\% of their MHR. Verbal coaching was given to help subjects stay within the target HR zone. All subjects were able to reach and sustain the target HR zone for the required time. Total exercise time ranged from 4 - 6 minutes. EIT data was not collected during the exercise phase. Subjects were then asked to return to the prone position as quickly as possible and assume their assigned posture, being one of: 1) a control posture with arms at the side (as in R and W), 2) hands clasping each other on the small of the back, or 3) both hands on the back of the head. The weight was quickly re-applied, and data was recorded for 5 minutes $\left(\mathrm{X}_{1}-\mathrm{X}_{5}\right)$. The weight was immediately removed, then subjects were recorded for another 2 minutes while maintaining the assigned posture $(\mathrm{P})$.

The forward model used for solving the internal conductivity distribution was based on the "adult_male" thorax shape in the EIDORS shape library. The lungs were segmented by overlaying the "adult_male_16el_lungs" model over the forward 

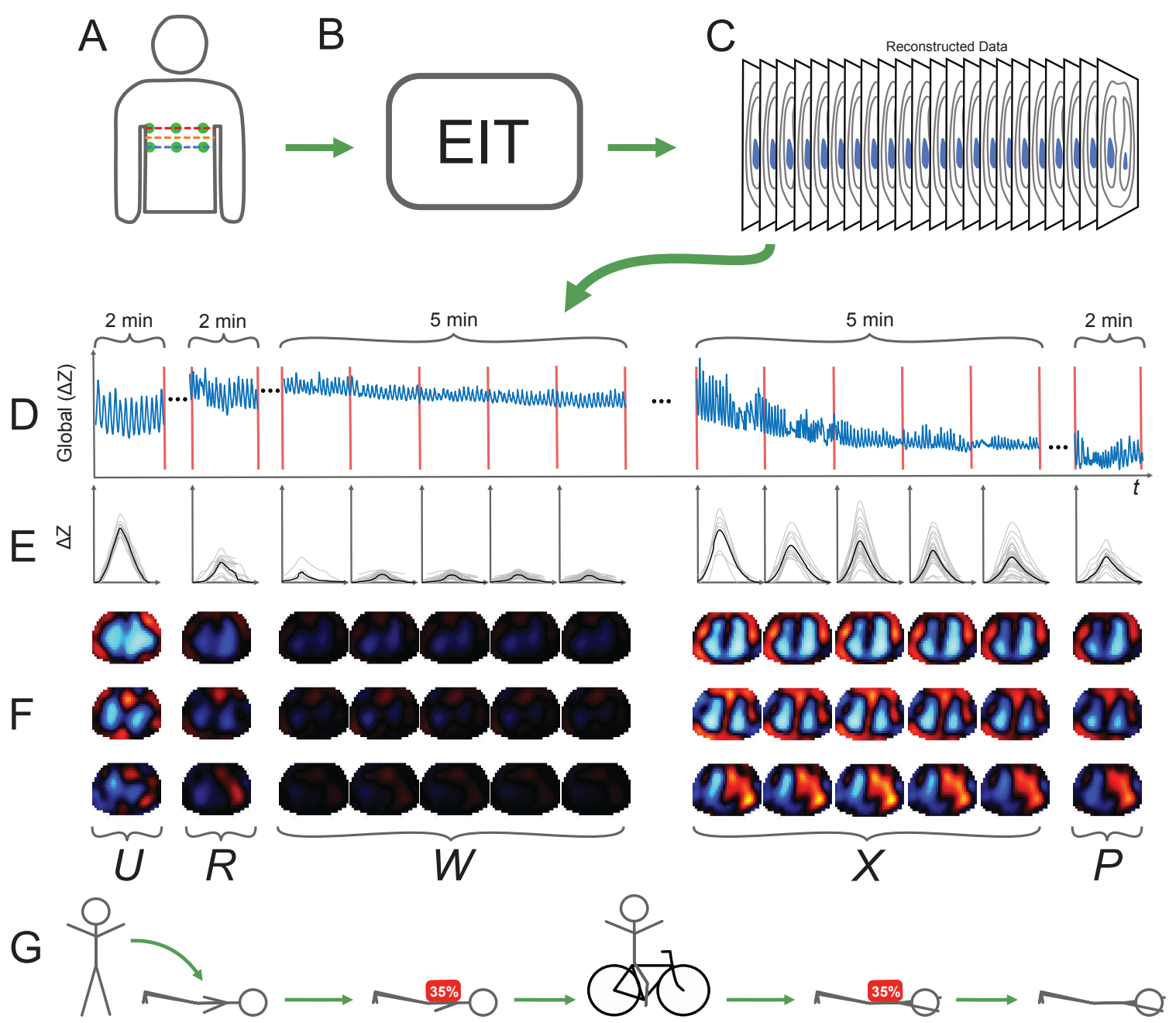

Figure A.1: Overview of experimental protocol. A) Two planes of EIT electrodes $(2 \times 16)$ were placed on the subject and connected to the SenTec EIT Pioneer Set in a square pattern. B) EIT measurements were recorded for 5 experimental phases: standing upright (U), unweighted prone $(\mathrm{R})$, weighted prone $(\mathrm{W})$, weighted prone in the restraint posture $(\mathrm{X})$, and unweighted prone in the restraint posture $(\mathrm{P})$. C) Conductivity values reconstructed from voltage data were obtained and used for calculation of parameters. Breath detection was applied on the global sum of $\Delta \mathrm{Z}$ values of each frame in each phase. D) The global pixel waveform is shown for a sample of each phase (weighted phases were divided into 1-minute periods). E) In each phase, breaths were detected (shown as thin lines) from the global $\Delta \mathrm{Z}$ and the average breath (bold line) was calculated. $\mathrm{F}$ ) $\mathrm{V}_{T}$ images (see text for details) were reconstructed for 3 planes (show in (A)) using the selected breaths shown in E. G) Schematic of experimental phases. The strenuous bicycle exercise task ( $\leq 10 \mathrm{~min})$ is illustrated between phases $\mathrm{W}$ and $\mathrm{X}$.

model.

EIT data was reconstructed in MATLAB R2019a using the EIDORS software package [63]. Data was lowpass filtered at $1 \mathrm{~Hz}$ using a $57^{\text {th }}$ order Kaiser window FIR 
filter. The first and last 50 frames of each time series were discarded to remove filter artifacts. Data from 2 participants were excluded due to noise contamination.

Breaths were defined by 3 points: the breath inspiration maximum and its two flanking expiration minima. A breath rejection algorithm was used to identify true breaths and reject noise from inadvertent talking or coughing. Parameters were then calculated from the averaged accepted breaths for each phase and sub-phase.

Three image planes, one at each electrode ring and one at the midpoint, were reconstructed using the GREIT 3D algorithm [86]. Images for each phase were reconstructed using the mean voltage of the first accepted breath as the reference. This scheme was chosen to eliminate the effect of changes in contact impedance due to perspiration, pressure exerted on the electrodes by prone positioning and/or the applied weight on voltage measurements.

Tidal volume $\left(\mathrm{V}_{T}\right)$ values were calculated from reconstructed images. For each accepted breath, a $\mathrm{V}_{T}$ image was produced from the conductivity difference $(\Delta \mathrm{Z})$ observed between its inspiration maximum and the mean of its two flanking expiration minima. Each $\mathrm{V}_{T}$ value was taken as the negative sum of the $\Delta \mathrm{Z}$ for all pixels within the lung segmentation of a single $\mathrm{V}_{T}$ image. The mean and standard deviation of the $\mathrm{V}_{T}$ values for a given period were then reported. The reconstructed images for an individual subject (as in figure A.2) show the mean value for each pixel across $V_{T}$ images for a given timeframe.

Instantaneous respiratory rate $(\mathrm{RR})$ in breaths $\cdot \mathrm{min}^{-1}$ was calculated for each breath using the inverse length of time between expiration minima. RR was expressed as the average value of breaths occurring during that phase or sub-phase, then expressed as a ratio of $R R$ in phase $R$. The reconstructed images for phase $R$ were averaged into a single image. In each of the $\mathrm{W}$ and $\mathrm{X}$ phases, 5 images were produced as the average of accepted breaths occurring in 5 non-overlapping $60 \mathrm{~s}$ win- 
dows.

A new parameter, representing the changes in functional residual capacity $(\triangle \mathrm{FRC})$, was used to quantify the relative amount of air in an individual's lungs at end expiration compared to the beginning of a phase. Comparisons between different phases for this value represent differences in reserve volume change, rather than differences in absolute lung reserve volume. $\triangle \mathrm{FRC}$ values were calculated from reconstructed images for each accepted breath. The mean of the negative sums of all pixels in the lung segmentation at the two expiration minima for each accepted breath was first calculated. The difference between a given mean end expiratory value and the first mean end expiratory value for a phase was the $\triangle \mathrm{FRC}$. This difference value was then converted to a relative volume by expressing it as a percentage of the mean $V_{T}$ of phase $\mathrm{R}$.

Minute ventilation (MV) was calculated as the product of measured $\mathrm{RR}$ and $\mathrm{V}_{T}$ values, then expressed as a ratio of MV at phase $\mathrm{R}$. Center of ventilation (CoV) values were calculated as previously described [87] and are shown as white crosshairs in figure A.2.

\section{A.4 Results}

We analyzed minute ventilation $(\mathrm{MV})$, relative functional reserve volume $(\triangle \mathrm{FRC})$, and center of ventilation $(\mathrm{CoV})$ while subjects were prone, prone with weight, and prone with weight in one of three restraint postures after an exercise task. Data from 19 subjects was collected. The full protocol as outlined above was completed by 7 subjects. The remaining 12 subjects completed all phases except for phase P. Data from phase $U$ was rejected from analysis due to noise levels. The data from 2 subjects from the pool which had not completed phase $\mathrm{P}$ were excluded due to noise 
contamination. Physiological data for the 17 analyzed subjects whose are summarized in table A.1.

\begin{tabular}{lcccc} 
Group & Age & Height $(\mathrm{cm})$ & Weight $(\mathrm{kg})$ & BMI $\left(\mathrm{kg} \cdot \mathrm{m}^{-2}\right)$ \\
\hline All & $25.8 \pm 7.86$ & $177 \pm 6.15$ & $80.4 \pm 14.4$ & $25.7 \pm 3.92$ \\
\hline Control & $22.4 \pm 2.56$ & $180 \pm 5.58$ & $79.5 \pm 10.6$ & $24.4 \pm 3.01$ \\
\hline Posture 1 & $24.4 \pm 6.07$ & $176 \pm 4.16$ & $81.7 \pm 15.3$ & $26.2 \pm 3.93$ \\
\hline Posture 2 & $30.4 \pm 10.4$ & $173 \pm 6.32$ & $80.0 \pm 16.6$ & $26.4 \pm 4.37$
\end{tabular}

Table A.1: Physiological data for the full participant pool and for each restraint posture. BMI: body mass index.

The consensus among subjects was that the protocol was uncomfortable but tolerable. A detailed report of observations for 3 subjects that completed the full protocol, one in each posture, is shown in figure A.2. The overall results are summarized in table A.2. Significance levels were determined by 1-way ANOVA using a significance level of $\mathrm{p}<0.05$. 
A
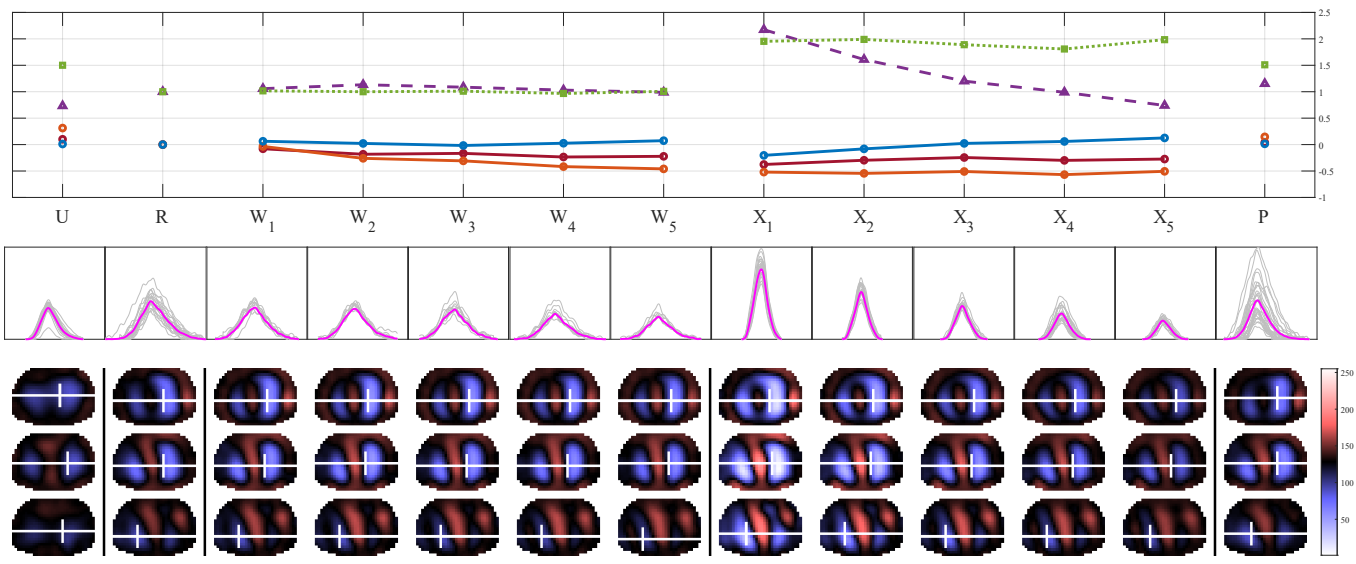

B
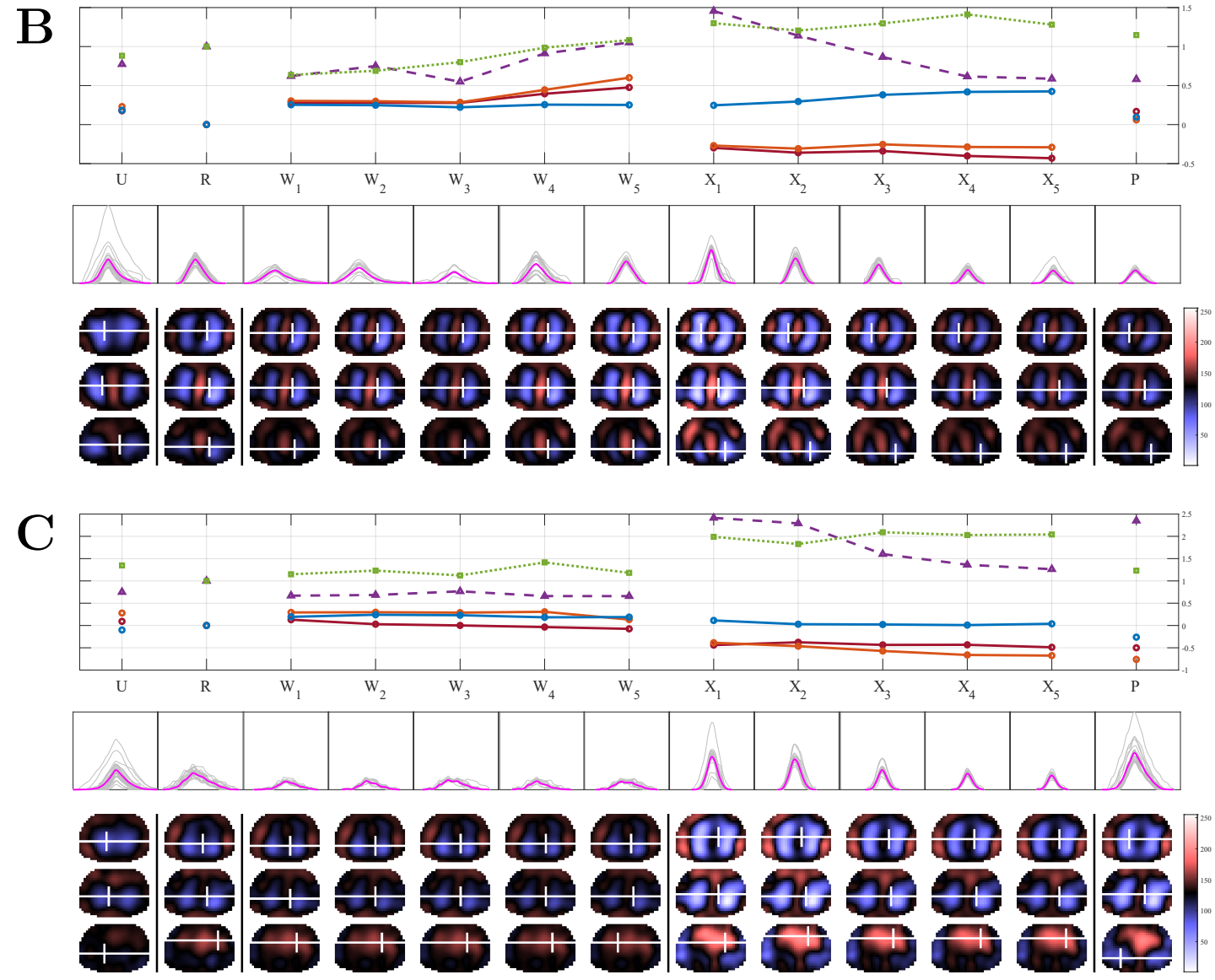

Figure A.2: Results for subject 7, 4, and 2, in the control (A), restraint 1 (B), and restraint 2 (C) postures, respectively. Experimental phases are labelled as in A.1. Each column in phases W and X were 1-minute periods, while phase $\mathrm{R}$ was a 2-minute period. Each figure panel contains: Top) plots of $\mathrm{V}_{T}$ (purple), $\triangle \mathrm{FRC}$ the top (red), middle (orange), and bottom (blue) reconstruction planes, and RR (green), for each phase. Middle) accepted breaths for each phase are plotted in gray along with the average breath in magenta. All breaths across phases within a subject were plotted on the same scale. Bottom) the internal conductivity distributions for 3 reconstruction planes for each phase, in anatomical position. Conductivity values were scaled across phases for each participant independently, with lower conductivity values correspond to larger volumes of air in the lungs. The center of ventilation for each reconstruction plane is shown with a white crosshair. 
All subjects had significantly lower $\triangle \mathrm{FRC}$ in the middle image plane in phase $\mathrm{U}$ compared to phase $\mathrm{R}$ as seen in table A.2 and the group average in figure A.4, but changes in MV were variable between these two phases.

Firstly, we hypothesized that addition of weight in phase $\mathrm{W}$ would be associated with a decrease in lung reserve volume. The results in table A.2 and figure A.4 showed an initial increase in $\triangle F R C$ in $W_{1}$ compared to $\mathrm{R}$ as subjects responded to the weight. By $\mathrm{W}_{5}$, changes $\triangle \mathrm{FRC}$ with respect to $\mathrm{R}$ were not significant. $\triangle \mathrm{FRC}$ changes in $\mathrm{W}_{5}$ with respect to $\mathrm{W}_{1}$ were also insignificant. The larger $\mathrm{SE}$ of the mean in $\mathrm{W}_{5}$ compared to $\mathrm{W}_{1}$ seen in figure A.4 suggested that $\triangle \mathrm{FRC}$ responses to the weight over time were variable. No significant $M V$ changes in $W_{1}$ or $W_{5}$ were observed with respect to $R$.

Secondly, we hypothesized that exercise combined with weight and prone restraint in phase $\mathrm{X}$ would be associated with $\triangle \mathrm{FRC}$ decreases, and that these changes would be more dramatic than those observed in phase W. We also hypothesized that restraint postures would be associated with lower MV's than the control posture. We observed that the $\triangle \mathrm{FRC}$ values in $\mathrm{X}_{1}$ were significantly greater than observed in $\mathrm{W}_{1}$ (table A.2). $\triangle \mathrm{FRC}$ was significantly lower at $\mathrm{X}_{5}$ for all postures compared to the group average at $W_{5}$ (table A.2). MV was significantly elevated for all postures in $\mathrm{X}_{1}$ compared to $\mathrm{W}_{1}$. $\mathrm{MV}$ at $\mathrm{X}_{5}$ remained elevated compared to the group average at $\mathrm{W}_{5}$ for the control posture and restraint posture 2 , but not restraint posture 1 (table A.2). MV was higher than control for restraint posture 2 but not restraint posture 1 (table A.2). MV decreased for all subjects in all postures through phase $\mathrm{X}$, though unexpectedly, MV for restraint posture 2 was significantly higher than restraint posture 1 and the control, potentially due to age-related effects.

Though the statistical power of this study was not strong enough to find $\triangle \mathrm{FRC}$ differences between $\mathrm{W}_{5}$ and $\mathrm{X}_{5}$ with respect to posture, Significant decreases between $\mathrm{W}_{5}$ and $\mathrm{X}_{5}$ were observed in the full subject pool. The decreasing $\Delta \mathrm{FRC}$ trends 


\begin{tabular}{llllll} 
Group & SS & df & MS & F & Prob $>$ F \\
\hline Relative MV & & & & & \\
\hline U vs R, all (16) & 0.05424 & 1 & 0.05424 & 0.68 & 0.4164 \\
\hline R vs W1, all & 0.03523 & 1 & 0.03523 & 0.5 & 0.4845 \\
\hline W1 vs W5, all & 0.19451 & 1 & 0.19451 & 2.03 & 0.1641 \\
\hline W5 vs X1, all & 4.1416 & 1 & 4.14163 & 9.6 & 0.004 \\
\hline W5 vs X5 & 4.0225 & 1 & 4.02251 & 10.61 & 0.0027 \\
\hline RP1 X5 vs control X5 & 0.07959 & 1 & 0.07959 & 0.84 & 0.3843 \\
\hline RP2 X5 vs control X5 & 3.29187 & 1 & 3.29187 & 5.56 & 0.0428 \\
\hline X5-W5 wrt Posture & 4.6397 & 2 & 2.31984 & 5.35 & 0.0188 \\
\hline X5-W5 wrt Age & 2.4452 & 1 & 2.44518 & 4.44 & 0.0524 \\
\hline X5-W5 wrt BMI & 1.5252 & 1 & 1.52523 & 2.49 & 0.1353 \\
\hline X5 vs P, all $(7)$ & 0.00206 & 1 & 0.00206 & 0 & 0.9566 \\
\hline Relative $\Delta$ FRC & & & & & \\
\hline U vs R, all (16) & 0.85823 & 1 & 0.85823 & 33.29 & $<0.0001$ \\
\hline R vs W1, all & 0.16221 & 1 & 0.16221 & 5.86 & 0.0213 \\
\hline W1 vs W5, all & 0.05126 & 1 & 0.05126 & 0.28 & 0.6002 \\
\hline W5-W1 wrt Age & 0.32025 & 1 & 0.32025 & 1.81 & 0.1986 \\
\hline W5-W1 wrt Age & 0.12965 & 1 & 0.12965 & 0.68 & 0.4214 \\
\hline W5 vs X5, all & 10.8108 & 1 & 10.8108 & 15 & 0.0005 \\
\hline RP1 X5 vs control X5 & 0.45579 & 1 & 0.45579 & 0.69 & 0.4277 \\
\hline RP2 X5 vs control X5 & 0.4653 & 1 & 0.46534 & 0.3 & 0.5973 \\
\hline X5-W5 wrt Posture & 2.0386 & 2 & 1.01928 & 1.13 & 0.3508 \\
\hline X5-W5 wrt Age & 4.0431 & 1 & 4.04307 & 5.71 & 0.0305 \\
\hline X5-W5 wrt BMI & 1.7753 & 1 & 1.77526 & 2.07 & 0.1712 \\
\hline X5 vs P, all 7 ) & 1.76434 & 1 & 1.76434 & 4.06 & 0.067 \\
\hline
\end{tabular}

Table A.2: Relationships between MV and $\triangle \mathrm{FRC}$ with respect to restraint posture, age, and BMI. wrt: with respect to. RP1: restraint posture 1 (hands behind the back). RP2: restraint posture 2 (hands on the back of the head). Control: control posture (arms at the side). All: the entire participant pool $(\mathrm{N}=17)$. $\mathrm{N}=16$ for phase $\mathrm{U}$ and $\mathrm{N}=7$ for phase $\mathrm{P}$.

observed in the restraint postures but not in the control postures suggested that the restraint postures contributed to these $\triangle \mathrm{FRC}$ trends. The relationship between MV of the 3 postures and their $\Delta \mathrm{FRC}$ 's indicated that $\Delta \mathrm{FRC}$ values were not correlated with those of MV. We also observed an effect of age but not BMI on the MV and $\Delta \mathrm{FRC}$ differences between $\mathrm{W}_{5}$ and $\mathrm{X}_{5}$. 


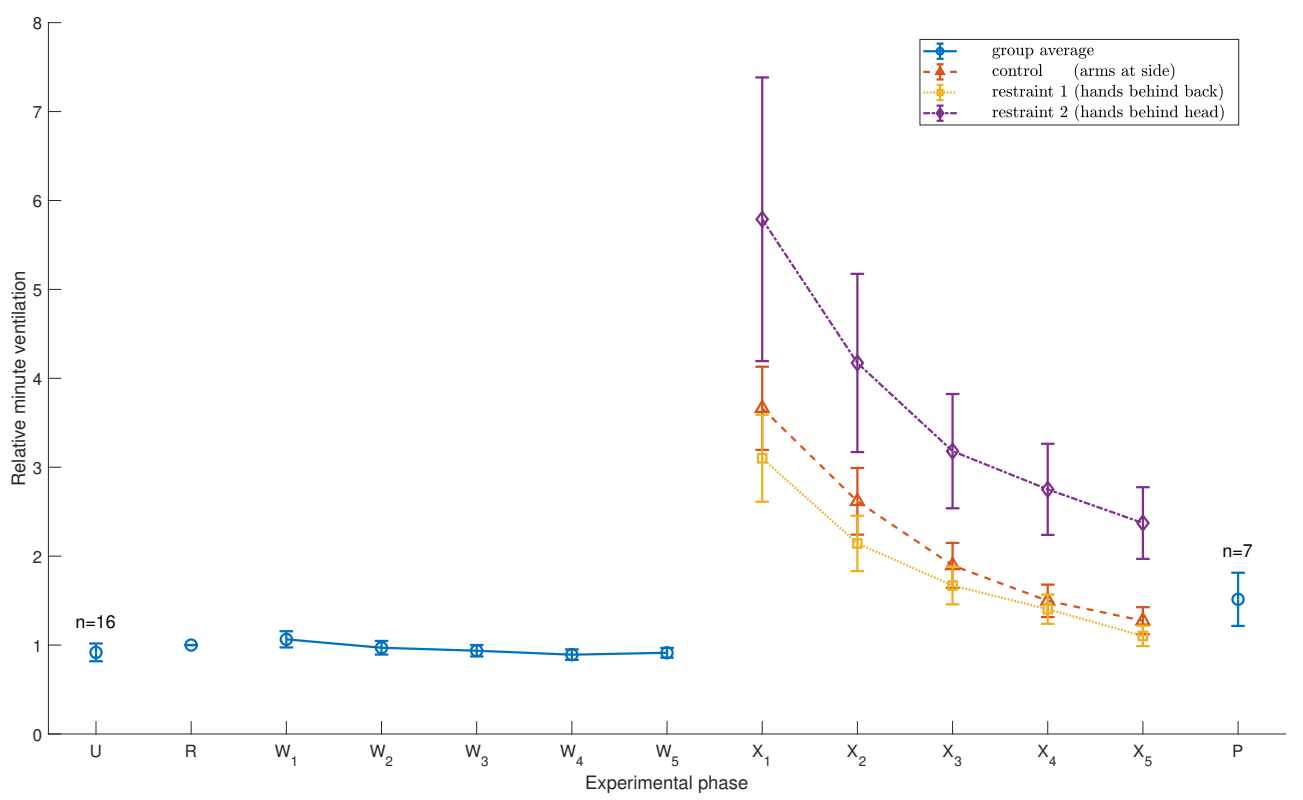

Figure A.3: Mean minute ventilation for each phase $\left(\mathrm{V}_{T} \cdot \mathrm{RR}\right)$, expressed as a ratio of the mean minute ventilation in phase $\mathrm{R}$, shown for the group average and each posture. Postures were either a control posture identical to that of phase $\mathrm{W}$, with arms at the side $(\mathrm{N}=5)$, or one of two restraint postures. Restraint posture 1: hands clasping each other on the small of the back $(\mathrm{N}=6)$. Restraint posture 2: both hands on the back of the head $(\mathrm{N}=6)$.

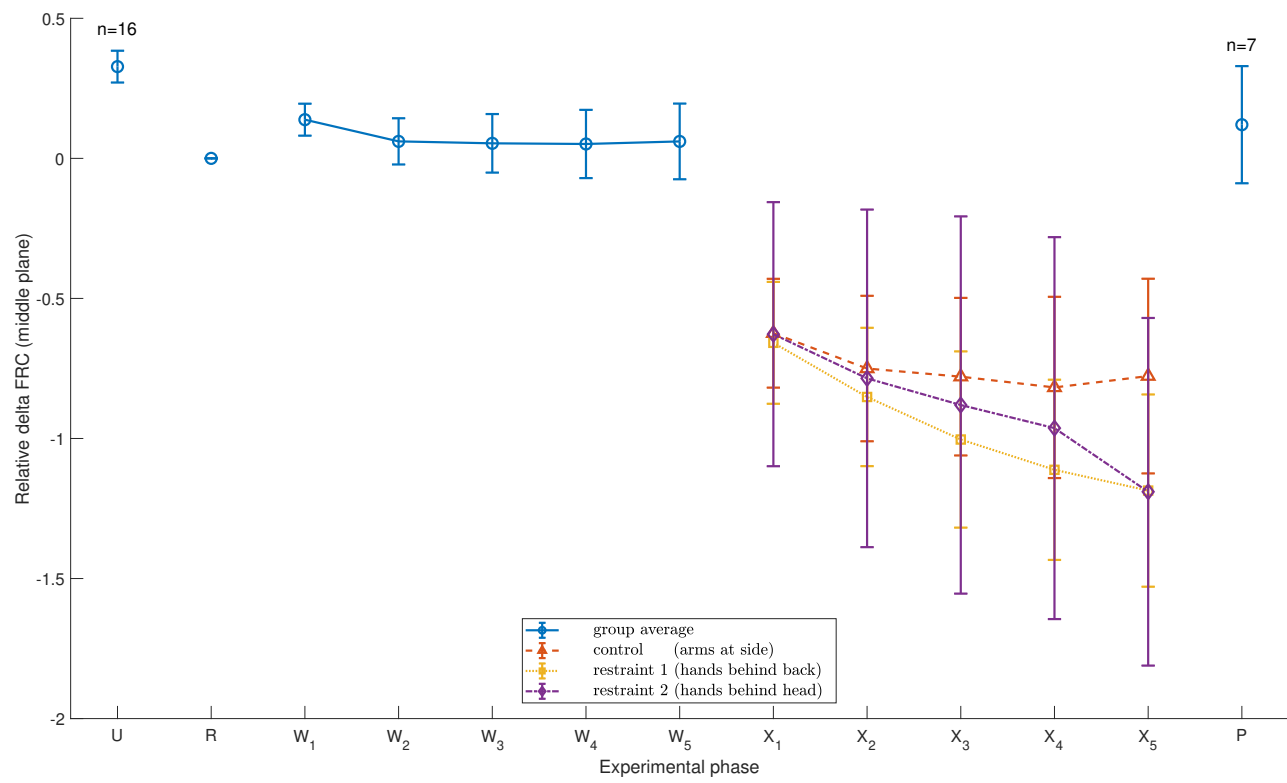

Figure A.4: Mean functional residual capacity change in the middle imaging plane from the onset of each phase, converted to a relative volume by expressing as a percentage of mean tidal volume in phase $\mathrm{R}$, and shown as a ratio of the change that occurred during phase $\mathrm{R}$, shown for the group average and each restraint posture. Postures were either a control posture identical to that of phase $\mathrm{W}$, with arms at the side $(\mathrm{N}=5)$, or one of two restraint postures. Restraint posture 1: hands clasping each other on the small of the back $(\mathrm{N}=6)$. Restraint posture 2: both hands on the back of the head $(\mathrm{N}=6)$. 


\section{A.5 Discussion}

In this study we developed a novel methodology for monitoring the cumulative effects of physical exertion, prone positioning, restraint, and body compression on respiration and ventilation distribution, for the first time, described in figure A.1. EIT electrodes were attached to subjects in a 3D imaging configuration, then reference measurements were taken while subjects were prone. The results of 17 subjects were analyzed.

When subjects transitioned from the standing to prone positions, decreases in $\Delta \mathrm{FRC}$ were observed that were consistent with previous reports using spirometry $[88,89]$. Overall, there was no significant change in MV from standing to prone positions, in agreement with changes observed by Moreno et al. in males between sitting and prone positions [88].

The addition of a $35 \%$ bodyweight external static load in phase $\mathrm{W}$ was associated with a $\triangle \mathrm{FRC}$ increase during the first minute $\left(\mathrm{W}_{1}\right)$, which was presumed to be an over-inflation of the lungs to counteract the external load. In the fifth minute $\left(\mathrm{W}_{5}\right)$, $\Delta \mathrm{FRC}$ was not significantly different than $\mathrm{R}$ or $\mathrm{W}_{1}$. The larger $\mathrm{SE}$ of the group mean at $\mathrm{W}_{5}$ compared to $\mathrm{W}_{1}$ suggested that $\triangle \mathrm{FRC}$ adaptations to the external load were initially consistent, but variable over time. $\triangle \mathrm{FRC}$ changes were not affected by subject BMI or age (table A.2), and occurred with no significant changes in MV throughout phase W. Given that the physiological response to a inspiratory resistive involves sympathetic nervous system activation and cardiac acceleration [90, 91], the $\Delta \mathrm{FRC}$ changes in $\mathrm{W}_{1}$ may have reflexive adaptations, which were later overridden on an individual basis for the remainder of phase $\mathrm{W}$. This variability suggests that the outward health of an individual cannot be taken as an indication of their response to control holds or body compression.

The effect of exercise and resisting an external inspiratory load has been previously 
shown to cause muscular fatigue and reduce an individual's ability to resist that load [92]. If there was no effect of body compression on MV and $\triangle \mathrm{FRC}$, then $\mathrm{MV}$ and $\Delta \mathrm{FRC}$ would be expected to gradually return to phase $\mathrm{R}$ levels as subjects recovered from exercise. Group MV was significantly elevated during the first minute of recovery after the exercise task $\left(\mathrm{X}_{1}\right)$ compared to $\mathrm{W}_{5}$. By $\mathrm{X}_{5}, \mathrm{MV}$ for all postures showed decreases indicative of recovery from exercise, but remained elevated with respect to $\mathrm{W}_{5}$ for all postures except restraint posture 2 . The analysis showed that there were significant postural effects on $\mathrm{MV}$ between $\mathrm{X}_{5}$ and $\mathrm{W}_{5}$ and near-significant age-related effects. The higher mean and standard deviation of age in restraint posture 2 may partially account for the higher MV and larger variability seen in this group for this phase.

As MV decreased while subjects recovered, $\triangle \mathrm{FRC}$ values for all subjects were lower than in $\mathrm{W}_{5}$ at both $\mathrm{X}_{1}$ and $\mathrm{X}_{5}$. This indicated that fatigue from the exercise task was responsible for this decrease. Though differences in $\Delta \mathrm{FRC}$ values at $\mathrm{X}_{5}$ were not significant with respect to posture, $\triangle \mathrm{FRC}$ in the control posture showed signs of stabilization and recovery, while $\triangle \mathrm{FRC}$ showed continued downward trends for both restraint postures. Furthermore, removal of the weight in phase $\mathrm{P}$ abolished this $\triangle \mathrm{FRC}$ decrease in $\mathrm{X} 5$, seen by increases in $\triangle \mathrm{FRC}$ for all but 1 of the 7 subjects recorded in phase $\mathrm{P}$ (table A.2 and figure A.4). Though dramatic, this change did not meet the level of significance due to this outlier.

The cumulative effects of prone positioning, body compression, physical exertion, and restraint on $\triangle \mathrm{FRC}$ observed in this study took place with an average applied weight of $28.1 \mathrm{~kg}$. This was less than the $56.8 \mathrm{~kg}$ and $90.9 \mathrm{~kg}$ weights used in [78] and less than the weight of an average person. In a live setting with potentially multiple officers applying their weight to a suspect, we expect that this reserve volume collapse could occur much earlier and be more dramatic than in our protocol. 
The normal recovery of MV observed in this study, taken alone, would not indicate respiratory vulnerabilities in these subjects. The detection of continued $\triangle \mathrm{FRC}$ decreases in the restraint postures by EIT which would have been overlooked by other methods demonstrates the value of EIT in studies of weighted restraint. Additionally, these parameters can be measured from surface electrodes and do not encumber a patient's breathing.

Our results suggested that the sudden loss of lung reserve volume during weighted restraint after vigorous exercise supports the role of weighted restraint in cases of restraint asphyxia or inexplicable ARDs. The fatigue of respiratory accessory muscles during weighted restraint places the diaphragm at higher risk of fatigue, making subjects more vulnerable to respiratory failure [92] and asphyxiation. Taken together, these results suggest that respiratory collapse from fatigue during weighted restraint is a plausible mechanism or trigger for cases of unexplained ARDs. 
Appendix B

Functional Neuroimaging of the N-back Working Memory Task with 3D Electrical Impedance Tomography REB Protocol Form 
This is the primary CUREB study submission form, to be used when none of the other submission forms, intended for more specialized categories of research, are suitable. If you have any doubt about which form to use, or for help in completing this form, please contact the Office of Research Ethics at ethics@carleton.ca or by phone: 6135202600 ext. 2517 (CUREB A) or ext. 4085 (CUREB B).

Please submit this form, and all accompanying documents, through cuResearch. For further instructions, go to https://carleton.ca/researchethics/submit-an-application/.

1. Title and Date

\section{A Project Title}

Title of Research Project (Detailed instructions, Example) Functional Neuroimaging of the N-back Working Memory Task with 3D Electrical Impedance Tomography

\section{B Submission Date}

Date of completion of this form. Update each time the form is revised. (Detailed instructions, Example)

$$
\text { 2019-07-11 }
$$

\section{C Attachments}

List documents included with this application (e.g. consent materials, invitations, permissions) (Detailed instructions, Example)

Consent Form, Supervisor-Sponsor Signature Form, Recruitment Email and Verbal Script

\section{Project Team}

2A Lead Researcher

\begin{tabular}{|l|l|}
\hline$\square$ & Academic or Library Staff \\
\hline$\square$ & Post-doctoral Fellow \\
\hline$\square$ & Graduate Student \\
\hline$\square$ & Undergraduate \\
\hline$\square$ & Other \\
\hline
\end{tabular}

2B Academic Supervisor $\square$ Same as lead researcher
Last name/First name

CAMPBELL, Mark

Official university (or other institution) email address

markacampbell@sce.carleton.ca

Department, faculty and institution (Detailed instructions, Example)

Department of Systems and Computer Engineering, Faculty of Engineering and Design

Academic supervisor(s) Last name/First name. (Note, the supervisor must be copied on all correspondence with CUREB.)

ADLER, Andy

THORNHILL, Rebecca

Official university (or other institution) email address:

adler@sce.carleton.ca

rthornhill@toh.ca

Department, faculty and institution (Detailed instructions, Example)

Research co-supervisor, professor in the Department of Systems and Computer Engineering, Faculty of Engineering and Design. 
Research co-supervisor, adjunct research professor in the Department of Systems and Computer Engineering, Faculty of Engineering and Design. Imaging scientist in the Department of Medical Imaging, The Ottawa Hospital.

2C Project Team Members No other team members
List the project team members: 1) Last name/First name 2) Email address 3) Role in project 4) Department and institution (Detailed instructions, Example)

\section{N/A}

3. Study Overview

3A Study Goal
What research question(s) will this study seek to answer (1-2 sentences)? (Detailed instructions, Example)

To what extent can electrical impedance tomography (EIT) be used as a functional neuroimaging tool?

\section{B Study Purpose and Benefits}

\section{C Participant Interactions Overview}

Form Version: April 2019
Study rationale: why should the research be pursued; what are the benefits, and to whom? (Benefits can be to research community, companies, or society in general.) (Detailed instructions, Example)

- The development of EIT as a functional neuroimaging tool could allow researchers to probe deep brain regions that are inaccessible to EEG, in addition to surface regions.

- EIT systems are inexpensive, portable, and do not emit ionizing radiation. This makes EIT suitable for long-term monitoring. Potential applications include but are not limited to: monitoring a patient's state of anesthesia during surgery, identifying brain regions having seizure activity in persons living with epilepsy, monitor neuroplastic remodelling of the brain after stroke or concussion, and providing a low-cost brain imaging platform for hobbyists or app developers.

Briefly describe what will happen to, or will be required of, the participants during the research. (Only a project overview is required). (Detailed instructions, Example)

- Participants will be recruited by word of mouth using the material from the recruitment email and verbal script.

- Eligible participants will be scheduled for an in-person meeting.

In this meeting, the protocol will be explained, and the participants will have an opportunity to ask questions. The researcher will ask for verbal consent from participants and record the date and time that consent was given. The data that will be collected will be anonymous, so no signature or otherwise identifying information will be collected.

- The participants will be asked to sit in a chair and wear a 32electrode head cap. Conductive gel will be applied between each electrode and the participant's scalp.

- The participants will be asked to daydream for 10 minutes in order to collect information on their brain's resting-state activity.

- Before the first exercise and in between exercises, participants will be reminded that these exercises are designed to be difficult. If at any time they are feeling fatigued, stressed, or uncomfortable during the exercise, they are encouraged to skip 
questions or take breaks as needed. They will be asked to avoid pressing the mouse button if they are taking a break.

- Task-positive data will then be collected by asking the participants to complete three n-back working memory exercises $(n=1,2,3)$. The visual stimuli will be English words with neutral valence. Participants will be asked to press a mouse button every time the displayed word is the same as the word displayed ' $n$ ' times ago.

- Participants will have the opportunity to rest for long as needed in between each working memory exercise. After 2 minutes the researcher will ask the participant if they are ready to continue. If they need more time, the researcher will re-iterate that they may rest for long as they need and to let the researcher know when they are ready.

- Participants may leave once the protocol is complete or if they choose to withdraw their consent.

3D Minimal Risk Review Request

\begin{tabular}{|l|l|}
\hline$\square$ & Yes, minimal risk review \\
\hline$\square$ & No, not minimal risk \\
\hline
\end{tabular}

Should this protocol be considered for minimal risk review? If so, please briefly justify. If not requesting a minimal risk review, leave this section blank. (CUREB will decide whether an application is reviewed at full board or via a delegated process). (Detailed instructions, Example)

- The imaging modality poses very low risk to participants (ISO 10601-1). It has been used at Carleton for 10 years, as well as by other groups, such as Dr. Holder's group at UCL, on hundreds of subjects with no reported ill effects. EIT uses ECG-type electrodes that are placed on the skin and applies small, imperceptible electrical currents between electrodes.

- Participants will be seated indoors in a secure, climatecontrolled room throughout the experiment and will not be exposed to any risk of physical, emotional, mental, social, or economic harm that exceeds that experienced in everyday life.

- The cognitive strain experienced by participants during the $\mathrm{n}$ back exercise is not expected to exceed the level experienced during strong engagement in a game or work-related task. - Visual stimuli will be presented for $1 \mathrm{~s}$ followed by $1 \mathrm{~s}$ of a fixation cross. The researcher posits that the rate of visual stimulus presentation poses minimal risk of triggering seizures in participants that may suffer from photosensitive epilepsy.

\section{E Dates of} Recruitment/Participant Interaction
Estimated date when will you will start recruiting participants? (YYYY-MM-DD)

\section{9-09-16}

Estimated date when you will end participant interactions? (YYYYMM-DD) (Detailed instructions, Example)

2019-12-09

\section{F Additional Reviews}

\begin{tabular}{|l|l|}
\hline$\square$ & No additional review \\
\hline$\square$ & Departmental review \\
\hline
\end{tabular}

Has this project been reviewed for academic merit? (not required, but for the Board's information) By whom? (e.g. a Tri-Council grant application or student's thesis committee) (Detailed instructions, Example) 

using a separate sample of control participants, describe this group. (Detailed instructions, Example)

- Participants must be physically able to complete the protocol (reading words displayed on a screen and clicking a button) to be included in this study.

- Participants must give verbal consent to be included in this study.

- Participants will be excluded based on the following pre-existing conditions: Surgically implanted metal plates or subjects (such as those used in reconstructive surgery) in the head.

- Participants will be excluded from the study if they have a history of traumatic head injury, concussion, or stroke.

- Each participant's resting-state activity will be used as the baseline control for the $n$-back exercise.

\section{B Number of Participants (Sample size)}

How many participants will be recruited? If multiple groups of participants are involved, breakdown by participant type. Provide a justification including a statistical rationale if appropriate. (Detailed instructions, Example)

It is estimated that 10 participants will be recruited. The number of participants required will be updated with statistical rationale once computer simulations showing the expected signal strength in regions of interest relative to baseline have been completed.

\section{C Vulnerable Population} Not Vulnerable Population
Describe any vulnerabilities of the participant group(s) that may compromise their ability to give free and informed consent or cause additional risks. Describe your mitigation strategy to ensure valid consent. (Detailed instructions, Example)

There will be no power relationship between the researcher and potential participants that would influence their willingness to participate in this study.

\section{D Participant Relationship to} Researcher

\begin{tabular}{|l|l|}
\hline$\square$ & No previous relationship \\
\hline$\square$ & Instructor-Student \\
\hline$\square$ & Client \\
\hline$\square$ & Employee \\
\hline$\square$ & Friends/Family \\
\hline$\square$ & Participated in previous \\
\hline & study \\
\hline$\square$ & Other \\
\hline
\end{tabular}

Describe any relationship that exists between the participants and the research team or any recruiting party or sponsor. Indicate how relationships will be managed so there is no undue pressure on participants. (Detailed instructions, Example)

- Participants will be recruited by word of mouth, drawing from interested friends, family, and colleagues.

- Individuals will be made fully aware by the researcher that their participation is completely voluntary and that they may withdraw their consent at any time without consequence. 
4E Benefits to Participants

No Direct Benefits
Describe any potential direct benefits to the research participants as opposed to society or knowledge. (Detailed instructions, Example)

- Individual-level functional neuroimaging data will not be available to participants.
4F Benefits to Participant Community

No Direct Benefits
Describe any benefits to your research participant community (e.g. indigenous community), such as capacity building, knowledge sharing, and fulfillment of community research priorities. (Detailed instructions, Example)

This research has no direct benefit to the research participant community.

Describe any conflicts of interest, and indicate how they will be managed. (Detailed instructions, Example) The researcher has no conflicts of interest.
4H Researcher Training with Participant Group

Not applicable
In addition to the TCPS2 training, describe any additional training the researcher(s) have (or will receive) to work with the proposed participants (e.g. research with Indigenous communities). (Detailed instructions, Example, TCPS2 Training)

The researcher has first aid training and specific training with the EIT system being used.

\section{Indigenous Peoples and Community Engagement}

\section{A Research involving Indigenous/Aboriginal peoples}

If none of the statements are applicable, skip this section (Detailed instructions, Example)

$\square$ Recruitment criteria includes Indigenous identity as a significant factor

$\square$ Study will seek input from participants regarding Indigenous communities, cultures, artifacts, traditional knowledge or unique characteristics

$\square$ Indigenous identity or membership in an Indigenous community is a factor in data analysis (e.g. sub-group analysis)

$\square$ Interpretation of the research findings will refer to Indigenous communities, peoples, languages, histories or cultures

\section{B Consultation}

Describe the consultation process with the indigenous community/ies. What is the community's involvement in governance of the research? With whom did you consult and what arrangements, if any, were made to implement Tri-Council (TCPS 2 Chapter 9) principles? If no consultation has taken place, please explain. (Detailed instructions, Example)

\section{N/A}


5C Approvals/Agreements
As part of the above process, describe what approvals/agreements you have made with the participating community/ies. (Detailed instructions, Example)

N/A

\section{D Benefits to Participant Community}

Describe how the research will provide fair benefits to the participating community/ies, meet community research priorities, support capacity building through enhancement of the skills of community personnel, and recognize the role of elders and other knowledge holders. (Detailed instructions, Example)

N/A

\section{E Participant involvement in research findings}

Describe how participants will be given the opportunity to participate in the interpretation of the data and review of research findings prior to the completion of any reports or publications? If such participation will not occur, explain. (Detailed instructions, Example)

$\mathrm{N} / \mathrm{A}$

\section{F Data Ownership, Control. Access and Possession}

Describe arrangements for the participating community's/ies' ownership and/or sharing of project data and findings, including the OCAP principles (ownership, control, access and possession).

\section{$6 . \quad$ Methods: Recruitment}

\section{A Recruitment Methods}

\begin{tabular}{|l|l|}
\hline$\square$ & Not applicable \\
\hline$\square$ & Posters \\
\hline$\square$ & Social Media \\
\hline$\square$ & Online Panels (e.g. \\
& Qualtrics) \\
\hline$\square$ & Student Participant Pool \\
(e.g. SONA)
\end{tabular}

Describe each step of how participants will be recruited. This includes how prospective participants will be identified, how contact information will be obtained, how participants will be made aware of the study, and how participants can express their interest. Provide a copy of all the recruitment material(s) including any oral scripts, recruitment posters, recruitment emails, social media postings etc. (Detailed instructions, Example)

- Participants will be recruited by word of mouth.

- Any person meeting the inclusion/exclusion criteria specified in 4A will be eligible for participation.

- Please see the included recruitment email and verbal script

\section{B Location of Recruitment}

\begin{tabular}{|l|l|}
\hline$\square$ & Not applicable \\
\hline$\square$ & Carleton \\
\hline$\square$ & Other Canadian \\
\hline & School/University \\
\hline
\end{tabular}

List all recruitment locations. If some locations require permission prior to recruitment, indicate if permission has been secured. (Detailed instructions, Example) Recruitment will take place in Ottawa by word of mouth. 


\begin{tabular}{|l|l|}
\hline$\square$ & Canada \\
\hline$\square$ & Online \\
\hline$\square$ & Other \\
\hline
\end{tabular}

6C Third Parties in Recruitment Not applicable
If using third parties to recruit, indicate who is doing the recruitment and how it will be accomplished. Does the third party have the prospective participant contact information? Are community leaders involved in identifying potential participants? (Detailed instructions, Example)

$$
\mathrm{N} / \mathrm{A}
$$

Describe any risks to participants during the recruitment phase, including risks to privacy. (Detailed instructions, Example)

No risks.

\section{E Recruitment risks to Researcher}

No risks

\section{F Compensation}

\begin{tabular}{|l||l|}
\hline$\square$ & No Compensation \\
\hline$\square$ & Money / Gift Card \\
\hline$\square$ & Reimbursement of Travel \\
\hline$\square$ & Expenses \\
\hline$\square$ & Refreshments \\
\hline$\square$ & Course Credit \\
\hline$\square$ & Other \\
\hline
\end{tabular}

\section{Methods: Informed Consent}

7A Obtaining informed consent

\begin{tabular}{|l|l|}
\hline$\square$ & Signed consent \\
\hline$\square$ & Online consent \\
\hline$\square$ & Oral consent \\
\hline$\square$ & Implied consent \\
\hline$\square$ & Parent/Guardian consent \\
\hline$\square$ & Assent \\
\hline$\square$ & Other \\
\hline
\end{tabular}

\section{B Deception}

Form Version: April 2019
Describe any risks to the research team during the recruitment phase. (Detailed instructions, Example)

No risks.

Describe all participant compensation and remuneration (including its monetary value) and indicate when participants will receive the compensation. What happens to the compensation if a participant withdraws? (Detailed instructions, Example) Participants will receive a $\$ 10$ gift card to Tim Horton's for their time as well as reimbursement for travel and/or parking costs.

Describe the process for obtaining informed consent from the participants (or guardians/legal representatives). If written consent is not used, explain the alternative method chosen. Include a copy of all consent forms, scripts and other materials. (Detailed instructions, Example)

Each participant will be asked to give verbal consent after they have read the consent form and had all of their questions about the protocol answered. 


\begin{tabular}{|l|l|}
\hline$\bigotimes$ & $\begin{array}{l}\text { Full Disclosure (i.e. no } \\
\text { deception) }\end{array}$ \\
\hline \hline$\square$ & Partial Disclosure \\
\hline \hline$\square$ & Mild Deception \\
\hline \hline$\square$ & More than Mild Deception \\
\hline
\end{tabular}

secondary consent and include forms or text. (Detailed instructions, Example)

There will be no deception in this protocol.

\section{C Debriefing}

Not required

Describe if, when, and how participants will be debriefed. (Include a copy of any documents that will be provided to participants). Describe any risks during debriefing and how they will be mitigated. (Detailed instructions, Example)

A debrief will not be required because there will be no deception in the protocol.

\section{D Withdrawal Procedures}

\begin{tabular}{|l|l|}
\hline$\square$ & Not applicable \\
\hline$\square$ & Participants can withdraw \\
\hline \hline$\square$ & $\begin{array}{l}\text { Participants can only } \\
\text { withdraw during the study } \\
\text { session }\end{array}$ \\
\hline$\square$ & $\begin{array}{l}\text { Special withdrawal } \\
\text { procedures }\end{array}$ \\
\hline$\square$ & $\begin{array}{l}\text { Full compensation to } \\
\text { withdrawn participants }\end{array}$ \\
\hline
\end{tabular}

8. Methods: Data Collection

8A Data Collection Methods

\begin{tabular}{|l|l|}
\hline$\square$ & Questionnaires / Surveys \\
\hline \hline$\square$ & Interviews \\
\hline$\square$ & Focus Groups \\
\hline$\square$ & Oral and/or Visual Stimuli \\
\hline$\square$ & $\begin{array}{l}\text { Equipment and/or software } \\
\text { testing }\end{array}$ \\
\hline \hline$\square$ & Other \\
\hline
\end{tabular}

Describe the procedures for a participant to withdraw. What will happen to data from participants who withdraw? Describe any deadlines and limitations on withdrawal, during the study or after research participation is complete. Explain if compensation amount is affected by withdrawal. (Detailed instructions, Example) Participants may withdraw from the study during the time when they are on campus with the researcher. Once consent is withdrawn, the experiment will end. Participants will be asked whether the data that has already been collected from them can be used in the study. If they want the data to be removed, we will delete the files.

Participants will be made aware that we will be unable to remove their data from the study after their date of participation because the data will be collected anonymously, and their files will not be identifiable.

Participants will still receive compensation if they choose to withdraw after they have given consent.
Describe in detail the method of data collection being used and provide details of any instruments used. Breakdown by phases, participant groups, or types if required. Complete the section on "online data collection" if relevant. (Fully describe or include a copy of any questionnaires, surveys, interview guides, or other data collection instruments). (Detailed instructions, Example)

1. Participants will arrive in the lab.

2. The researcher will give a brief overview of the research and introduce the participant to the equipment that will be used.

3. Participants will be given the consent form to read, which will describe the protocol in full detail. The consent form will also describe the exclusion criteria. The participants will be asked to notify the researcher if they meet any of the exclusion criteria, but they will not be asked to give any details of which criteria they have met. 
4. The researcher will explain to participants that the collected data will be completely anonymous. Participants will not be able to remove their data from the study after their date of participation because it will not be identifiable. Participants will be asked for their verbal consent to participate.

5. Participants will be asked to wear a commercially available 32-electrode EEG headcap (not yet sourced). Conductive gel will be applied between each electrode and the participant's scalp.

6. The Swisstom EIT data acquisition set will be used to collect and transfer data to a secure laptop.

7. Visual stimuli will be presented to the participants via a lab desktop monitor.

8. Participants will click a mouse button in response to visual stimuli.

9. Participants will have the opportunity to rest for long as needed in between each working memory exercise. After 2 minutes the researcher will ask the participant if they are ready to continue. If they need more time, the researcher will re-iterate that they may rest for long as they need and to let the researcher know when they are ready.

10. Once the exercises are complete, the researcher will remove the electrodes from the cap, remove the cap from the participant's head, and offer to clean off the conductive gel. A wash station with a sink and towels will be available to participants for further cleaning.

11. Participants will be thanked for their time and remunerated with a $\$ 10$ Tim Hortons gift card and told that they are free to leave.

8B Location of Participant Interactions

\begin{tabular}{|l|l|}
\hline$\square$ & Carleton \\
\hline$\square$ & Workplace \\
\hline$\square$ & Public venue \\
\hline$\square$ & Online \\
\hline$\square$ & Outside Canada \\
\hline \hline$\square$ & Other \\
\hline
\end{tabular}

Where will the research procedures involving participants take place? (Detailed instructions, Example)

\section{Carleton University in lab CB 6107.}

\section{C Frequency and Duration of Participant Interactions}

How many times will you interact with participants? How long will each interaction take? (Detailed instructions, Example) The protocol will require one interaction of 75 minutes.
8D Photography or Recordings

\begin{tabular}{|l|l|}
\hline$\square$ & Not applicable \\
\hline$\square$ & Photographs \\
\hline
\end{tabular}

If the participant will be photographed, video-recorded or audiorecorded, indicate how the data will be acquired and protected. How will consent for recordings be obtained? If other (e.g. fingerprints or eye-tracking) please describe. Can participants opt 


\begin{tabular}{|l|l|}
\hline$\square$ & Audio Recording \\
\hline$\square$ & Video Recording \\
\hline$\square$ & Other (Please describe) \\
\hline
\end{tabular}

out of recordings and still participate? (Detailed instructions, Example)

N/A

8E Translation or Transcription

\begin{tabular}{|l|l|}
\hline$\square$ & Not applicable \\
\hline \hline$\square$ & Translation \\
\hline \hline$\square$ & Transcription \\
\hline$\square$ & $\begin{array}{l}\text { Researcher will translate or } \\
\text { transcribe }\end{array}$ \\
\hline
\end{tabular}

If you require the services of a translator or transcriber, describe what services you will use and how you will interact with the translator and/or transcriber. If a confidentiality agreement will be used, include a copy. (Detailed instructions, Example) N/A
8F Online data collection

\begin{tabular}{|c|c|}
\hline 叉 & Not applicable \\
\hline$\square$ & Carleton-based server \\
\hline$\square$ & $\begin{array}{l}\text { Commercial server (based } \\
\text { in Canada) }\end{array}$ \\
\hline$\square$ & $\begin{array}{l}\text { Commercial server (outside } \\
\text { Canada) }\end{array}$ \\
\hline$\square$ & Other \\
\hline
\end{tabular}

Describe the software platform used for online data collection, and the security of data storage. Where will data be stored? Will participant IP addresses be recorded? Are there any special limitations on privacy? (Detailed instructions, Example)

No data will be collected online.

\section{$8 G$ Biological specimens or fluids}

\} \text { Not applicable }
Describe the apparatus and methods to collect biological specimens or fluids (e.g., blood, saliva, tissue samples). How will specimens be stored? If any will be retained or transferred to another institution/research group, explain the research purpose, and plans for eventual destruction, if any. (Detailed instructions, Example)

\section{$\mathrm{N} / \mathrm{A}$}

Describe any drugs, devices or diagnostic apparatus being studied or used, or any physical or physically intrusive research interventions, such as sending energy into the body (e.g. electrodes, MRI/X-ray), or physiological activities (e.g. exercise or stress). Explain any risks to the participants and compare the dose to established safety standards. (Detailed instructions, Example)

EIT involves the injection of harmless electrical currents ranging from 0.1 to $0.5 \mathrm{~mA}$ at $\sim 50 \mathrm{kHz}$ between pairs of electrodes on the surface of the skin. EIT poses minimal risk to participants.

The participant will be asked to wear the electrode headcap, through which the currents will be automatically applied. Voltage measurements from non-injecting electrodes will be taken and recorded in a data file.

EIT measurements of each participant's brain, and motor response to visual stimuli will be recorded. This data will be 
completely anonymous, and only labelled with the participant's handedness. Data will be stored on a secure laptop.

8I Risk of Psychological Harm $\unrhd$ No risks
Explain the nature, magnitude and probability of these risks and how they will be mitigated. (Detailed instructions, Example)
8J Risk of Physical Harm No risks

8K Risk of Social and/or Economic Harm

$\triangle$ No risks

8L Incidental Findings Incidental findings unlikely
Explain the nature, magnitude and probability of these risks and how they will be mitigated. (Detailed instructions, Example)

Explain the nature, magnitude and probability of these risks and how they will be mitigated. (Detailed instructions, Example)

Describe possible incidental findings and how they will be managed (e.g. becoming aware of abuse of a child, imminent harm to a participant or third party, or potentially significant clinical findings). Any resulting limitations of confidentiality should be communicated to participants. (Detailed instructions, Example)

\section{Methods: Data Storage and Analysis}

9A Identifiability of collected data

\begin{tabular}{|l|l|}
\hline$\square$ & Identifiable \\
\hline$\square$ & Coded (pseudonyms) \\
\hline \hline & Anonymous \\
\hline$\square$ & Other \\
\hline
\end{tabular}

Describe the identifiability of research data at the point of data collection. If there are different levels of anonymity for different groups, describe. (Detailed instructions, Example)

Collected data will be anonymous.
9B Identifiability of stored data

\begin{tabular}{|l|l|}
\hline$\square$ & Identifiable \\
\hline$\square$ & Coded (pseudonyms) \\
\hline$\square$ & Anonymous/anonymized \\
\hline$\square$ & Other \\
\hline
\end{tabular}

Describe the identifiability of stored research data. If a link to participant identities is retained (e.g. to permit compensation or withdrawal), also explain storage of linking data. Describe the process of anonymization if applicable. (Detailed instructions, Example)

Stored research data will be anonymous.
9C Identifiability of published data

\begin{tabular}{|l|l|}
\hline$\square$ & Anonymous \\
\hline$\square$ & Aggregate data only \\
\hline$\square$ & Pseudonyms/Coded \\
\hline
\end{tabular}

Describe the identifiability of data that will appear in publications, including how pseudonyms will be assigned, if applicable. If there are different levels of anonymity for different groups, describe each level here. (Detailed instructions, Example)

Published results and discussion will be anonymous. 
$\square$ Real participant names with data attributable

$\square$ Other

9D Data Storage (during the project)

\begin{tabular}{|l|l|}
\hline$\square$ & Encrypted \\
\hline$\square$ & Password-protected \\
\hline$\square$ & Physical documents \\
\hline$\square$ & Other \\
\hline
\end{tabular}

How will data be stored and kept safe? Provide details for each type of data (e.g. raw data, contact lists, consent documents, anonymized data, recordings and images, electronic data and paper documents). (Detailed instructions, Example)

- No identifying information will be collected. Electronic and physical correspondence between the researcher will be deleted or destroyed immediately after each participant's participation in the study.
9E Data Disposition (after the project)

$\otimes$ Stored

$\square$ De-identified data shared publicly

$\square$ Identifiable data shared publicly

$\square$ All identifiers/codes will be permanently deleted

$\square$ Returned to participants

$\square$ Destroyed
After project completion, describe whether and how the data will be stored for future use. If shared, with whom? If made public, how (e.g. online)? If archived, provide details. Describe any restrictions on access. Will personal identifiers be deleted and when? If data will be destroyed, when? Will participant contact information be kept for future recruitment? (Include data disposition plans in the consent materials) (Detailed instructions, Example)

The anonymous data will remain stored for future use.

\section{F Sharing Study Results}

$\bigotimes$ Results will be shared
Do you intend to share a report (or summary) of the research findings with participants once the study is complete? If yes, include this option in the consent form. (Detailed instructions, Example)

Participants may opt in to receiving an electronic copy of the final manuscript.

\section{G Data Breach Risks} $\unrhd$ No Risks
Describe the likelihood of a data breach and the resulting risks to participants. If risks are significant, how will they be mitigated? (Detailed instructions, Example)

The risk of a data breach is minimal. In the event of a data breach, participants will be protected by the anonymity of the data.

\section{Funding and Approvals}

\section{A Project Funding}

\begin{tabular}{|l|l|}
\hline$\square$ & Unfunded \\
\hline$\square$ & Tri-Council Funded \\
\hline$\square$ & Other Award/Grant \\
\hline$\square$ & Contract Funded \\
\hline
\end{tabular}

Who is funding this project? If applicable, include the funding source/agency/company, program, award name, and number (from CUResearch). Note if the researcher applied for a release of funds for this project funding.

NSERC Discovery Grant 
Personal Consulting or

Personal Work

Scholarship

\section{B Researcher Funding (for research contracts and personal consulting only) \\ Q Not contract funded research \\ $\square$ No funds are paid directly to the researcher as personal income \\ $\square$ The researcher will receive a portion of the funds as personal income \\ $\square$ A copy of the contract/agreement has been submitted to the Research Compliance Office}

For research that will pay personal income to any researcher: how will any resulting conflicts of interest be managed? How much funding (dollar amount and the percentage of the total) will the researcher(s) receive as income? Provide the title and date of any contracts. (The REB may review the contract.)

\section{C Additional Approvals} Required

\begin{tabular}{|l|l|}
\hline$\square$ & $\begin{array}{l}\text { No other approvals } \\
\text { required }\end{array}$ \\
\hline$\square$ & Organizational Permission \\
\hline$\square$ & Visa/Travel Permits \\
\hline$\square$ & Other REBs or Institutional \\
\hline & Approvals \\
\hline$\square$ & Biohazards \\
\hline$\square$ & Animal Care Committee \\
\hline$\square$ & Permission letters attached \\
\hline \hline$\square$ & Letters to follow \\
\hline$\square$ & Other (please specify) \\
\hline
\end{tabular}

Is organizational permission required to conduct research (e.g., schools, employers, other universities, correctional services, indigenous communities, or other data collection locations)? If conducting research in another country, is local permission, including local ethics review, required? Indicate if permission/approval has been secured and provide a copy. Research with biohazards or animals must also secure approval from the appropriate committee at Carleton University.

\section{D TCPS Tutorial}

\begin{tabular}{|l|l|}
\hline$\square$ & $\begin{array}{l}\text { lompleted the online TCPS } \\
\text { tutorial }\end{array}$ \\
\hline$\square$ & $\begin{array}{l}\text { Have not completed the } \\
\text { online TCPS tutorial }\end{array}$ \\
\hline
\end{tabular}

\section{Declarations}

\section{A Supervisor Approval}

$\square$ Not applicable

Form Version: April 2019
TCPS CORE Tutorial training is required for all researchers listed on the protocol. Justify any cases where researchers have not completed the TCPS tutorial.
For student projects, please indicate the date that the supervisor approved the application. Such approval indicates that the supervisor has read the entire submission and associated documentation, and is satisfied that the project is appropriately 
$\bigotimes$ Supervisor Approved

prepared and meets applicable disciplinary and ethical

standards. (Detailed instructions, Example)

2019-07-09

11B Declaration \# 1

\I agree
This ethics application accurately describes the research project or scholarly activity that I plan to conduct. (Detailed instructions, Example)
11C Declaration \#2 I I agree
No recruitment or data collection for this protocol will commence before ethics clearance. (Detailed instructions, Example)
11D Declaration \#3 I agree
No changes will be made to the research project as described in this protocol without receiving clearance from the Research Ethics Board. (Detailed instructions, Example)

11E Declaration \#4 II agree

12. Comments

12A Comments (optional)
The Research Ethics Board will be notified immediately of any alleged or real ethical breaches or concerns, adverse events, or participant complaints that arise during or after the course of this research project. (Detailed instructions, Example)
Do you have any comments or suggestions on the form? 


\section{References}

[1] E. Donkor, "Stroke in the 21st century: A snapshot of the burden, epidemiology, and quality of life," Stroke Res Treat, vol. 2018, no. 3238165, 2018.

[2] B. Tomandl, E. Klotz, R. Handschu, B. Stemper, F. Reinhardt, W. Huk, K. Eberhardt, and S. Fateh-Moghadam, "Comprehensive imaging of ischemic stroke with multisection ct," Radiographics, vol. 23, no. 3, pp. 565-92, 2003.

[3] L. Allen, A. Hasso, J. Handwerker, and H. Farid, "Sequence-specific mr imaging findings that are useful in dating ischemic stroke," Radiographics, vol. 32, no. 5, pp. 1285-1297, 2012.

[4] E. Marieb and K. Hoehn, Human Anatomy \&3 Physiology Eigth Edition. 1301 Sansome St., San Francisco, CA: Pearson Education Inc., 2010.

[5] D. Holder, "Electrical impedance tomography (eit) of brain function," Brain Topography, vol. 5, pp. 87-93, 1992.

[6] L. Enfield, Electrical impedance tomography of human brain function. PhD thesis, University College London, 2005.

[7] J. Latikka and H. Eskola, "The resistivity of human brain tumours in vivo," Ann Biomed Eng., vol. 47, no. 3, pp. 707-713, 2019. 
[8] Y. Li, D. Zhang, B. Liu, Z. Jin, W. Duan, X. Dong, F. Fu, S. Yu, and X. Shi, "Noninvasive cerebral imaging and monitoring using electrical impedance tomography during total aortic arch replacement," Journal of Cardiothoracic and Vascular Anesthesia, vol. 32, no. 6, pp. 2469 - 2476, 2018.

[9] B. Pollard, C. Pomfrett, A. Bryan, T. Quraishi, J. Davidson, and H. McCann, "Functional electrical impedance tomography by evoked response (feiter): Subsecond changes in brain function during induction of anaesthesia with propofol: 7ap1-6," European Journal of Anaesthesiology, vol. 28, pp. 97-98, 2011.

[10] D. Barber, B. Brown, and I. Freeston, "Imaging spatial distributions of resistivity using applied potential tomography," Electronics Letters, vol. 19, no. 22, pp. 933935, 1983.

[11] C. Putensen, B. Hentze, S. Muenster, and T. Muders1, "Electrical impedance tomography for cardio-pulmonary monitoring," J Clin Med, vol. 8, no. 8, p. 1176, 2019 .

[12] T. de Castro Martins, A. K. Sato, F. S. de Moura, E. D. L. B. de Camargo, O. L. Silva, T. B. R. Santos, Z. Zhao, K. Möeller, M. B. P. Amato, J. L. Mueller, R. G. Lima, and M. de Sales Guerra Tsuzuki, "A review of electrical impedance tomography in lung applications: Theory and algorithms for absolute images," Annual Reviews in Control, vol. 48, pp. 442 - 471, 2019.

[13] V. Cherepenin1, A. Karpov2, A. Korjenevsky1, V. Kornienko1, A. Mazaletskaya3, D. Mazourov4, and D. Meister5, "A 3d electrical impedance tomography (eit) system for breast cancer detection," Physiological Measurement, vol. 22, no. 1, pp. 9-18, 2001. 
[14] J. Borges, F. Suarez-Sipmann, S. Bohm, G. Tusman, A. Melo, E. Maripuu, M. Sandström, M. Park, E. Costa, G. Hedenstierna, and M. Amato, "Regional lung perfusion estimated by electrical impedance tomography in a piglet model of lung collapse," Journal of applied physiology (Bethesda, Md. : 1985), vol. 112, pp. 225-36, 092011.

[15] I. Frerichs, J. Hinz, P. Herrmann, G. Weisser, G. Hahn, M. Quintel, and G. Hellige, "Regional lung perfusion as determined by electrical impedance tomography in comparison with electron beam ct imaging," IEEE Transactions on Medical Imaging, vol. 21, no. 6, pp. 646-652, 2002.

[16] D. Nguyen, A. Bhaskaran, W. Chik, M. Barry, J. Pouliopoulos, R. Kosobrodov, C. Jin, T. Oh, A. Thiagalingam, and A. McEwan, "Perfusion redistribution after a pulmonaryembolism-like event with contrast enhanced eit," Physiological Measurement, vol. 36, pp. 1297-1309, 2015.

[17] A. Adler, M. Faulkner, K. Aristovich, S. Hannan, J. Avery, and D. Holder, "Cerebral perfusion imaging using eit," in Conf. EIT 2017, vol. 57, (Dartmouth, NH, USA), p. 44, Local Copy, Presentation, 2017.

[18] H. Schwan and C. Ferris, "Four-terminal null techniques for impedance measurements with high resolution," Rev. Sci. Instrum., vol. 39, pp. 481-485, 1968.

[19] C. Yang, S. Liu, Y. Feng, and H. Yang, "Influence of electrode polarization on the potential of dc electrical exploration," Journal of Applied Geophysics, vol. 149, pp. 63-76, 2018.

[20] A. Adler, J. H. Arnold, R. Bayford, A. Borsic, B. Brown, P. Dixon, T. J. C. Faes, I. Frerichs, H. Gagnon, Y. Gärber, B. Grychtol, G. Hahn, W. R. B. Lionheart, A. Malik, R. P. Patterson, J. Stocks, A. Tizzard, N. Weiler, and G. K. Wolf, 
"GREIT: a unified approach to 2d linear EIT reconstruction of lung images," Physiological Measurement, vol. 30, pp. S35-S55, jun 2009.

[21] C. Hansen, Discrete Inverse Problems: Insight and Algorithms. U.S.: Society for Industrial \& Applied Mathematics, 2010.

[22] K. Y. Aristovich, B. C. Packham, H. Koo, G. S. dos Santos, A. McEvoy, and D. S. Holder, "Imaging fast electrical activity in the brain with electrical impedance tomography," NeuroImage, vol. 124, pp. 204 - 213, 2016.

[23] S. Hannan, M. Faulkner, K. Aristovich, J. Avery, M. Walker, and D. Holder, "Imaging fast electrical activity in the brain during ictal epileptiform discharges with electrical impedance tomography," NeuroImage: Clinical, vol. 20, pp. 674 $-684,2018$.

[24] H. McCann, G. Pisano, and L. Beltrachini, "Variation in reported human head tissue electrical conductivity values," Brain Topography, vol. 32, no. 5, pp. 825858,2019

[25] D. Liang, S. Bhatta, V. Gerzanich, and J. Simard, "Cytotoxic edema: mechanisms of pathological cell swelling," Neurosurg Focus, vol. 22, no. 5, p. E2, 2007.

[26] A. Hansen and C. Olsen, "Brain extracellular space during spreading depression and ischemia," Acta Physiologica Scandinavica, vol. 108, no. 4, pp. 355-365, 1980.

[27] B. Lingwood, K. Dunster, P. Colditz, and L. Ward, "Noninvasive measurement of cerebral bioimpedance for detection of cerebral edema in the neonatal piglet," Brain Research, vol. 945, no. 1, pp. 97-105, 2002. 
[28] J. Avery, Improving Electrical Impedance Tomography of brain function with a novel servo-controlled electrode helmet. PhD thesis, University College London, 2015 .

[29] S. Nathan, S. Sinha, B. Gordon, R. Lesser, and N. Thakor, "Determination of current density distributions generated by electrical stimulation of the human cerebral cortex," Electroencephalography and Clinical Neurophysiology, vol. 86, no. 3, pp. 183-192, 1993.

[30] M. Joy, V. Lebedev, and J. Gati, "Imaging of current density and current pathways in rabbit brain during transcranial electrostimulation," IEEE Transactions on Biomedical Engineering, vol. 46, no. 9, pp. 1139-1149, 1999.

[31] H. Gamba and D. Delpy, "Measurement of electrical current density distribution within the tissues of the head by magnetic resonance imaging," Medical and Biological Engineering and Computing, vol. 36, pp. 165-170, 1998.

[32] A. Tidswell, A. Gibson, R. Bayford, and D. Holder, "Validation of a 3d reconstruction algorithm for eit of human brain function in a realistic head-shaped tank," Physiological Measurement, vol. 22, no. 1, pp. 177-185, 2001.

[33] T. Dowrick, C. Blochet, and D. Holder, "In vivo bioimpedance changes during haemorrhagic and ischaemic stroke in rats: towards $3 \mathrm{~d}$ stroke imaging using electrical impedance tomography," Physiological Measurement, vol. 37, pp. 765$784,2016$.

[34] A. S. Association, "Stopping the bleeding in a hemorrhagic stroke." https://www.stroke.org/en/about-stroke/treatment/hemorrhagic-stroketreatment, 2018. 2020-06-02. 
[35] J. Wardlaw, "Overview of cochrane thrombolysis meta-analysis," Neurology, vol. 57, no. suppl 2, pp. S69-S76, 2001.

[36] S.-L. Pai, R. D. Wang, and S. Aniskevich, "Perioperative stroke: Incidence, etiologic factors, and prevention," Minerva Anestesiol., vol. 83, no. 11, pp. 1178$1189,2017$.

[37] D. Holder, "Detection of cerebral ischaemia in the anaesthetised rat by impedance measurement with scalp electrodes: Implications for non-invasive imaging of stroke by electrical impedance tomography," Clinical Physics and Physiological Measurement, vol. 13, no. 1, pp. 63-75, 1992.

[38] A. McEwan, A. Romsauerova, R. Yerworth, L. Horesh, R. Bayford, and D. Holder, "Design and calibration of a compact multifrequency eit system for acute stroke imaging," Physiological Measurement, vol. 27, pp. S199-S210, 2006.

[39] A. Romsauerova, A. McEwan, and D. Holder, "Identification of a suitable current waveform for acute stroke imaging," Physiological Measurement, vol. 27, pp. S211-S219, 2006.

[40] T. Dowrick, C. Blochet, and D. Holder, "In vivo bioimpedance measurement of healthy and ischaemic rat brain: Implications for stroke imaging using electrical impedance tomography," Physiological Measurement, vol. 36, no. 6, pp. 1273$1282,2015$.

[41] L. Yang, W. Liu, R. Chen, G. Zhang, W. Li, F. Fu, and X. Dong, "In vivo bioimpedance spectroscopy characterization of healthy, hemorrhagic and ischemic rabbit brain within 10 hz-1 mhz," Sensors, vol. 17, no. 4, p. 791, 2017. 
[42] M. Dai, L. Wang, C. Xu, L. Li, G. Gao, and X. Dong, "Real-time imaging of subarachnoid hemorrhage in piglets with electrical impedance tomography," Physiological Measurement, vol. 31, pp. 1229-1239, 2010.

[43] M. Dai, B. Li, S. Hu, C. Xu, B. Yang, J. Li, F. Fu, Z. Fei, and X. Dong, "In vivo imaging of twist drill drainage for subdural hematoma: A clinical feasibility study on electrical impedance tomography for measuring intracranial bleeding in humans," PLoS ONE, vol. 8, no. 1, p. e55020, 2013.

[44] T. Tang, M. Weiss, P. Borum, S. Turovets, D. Tucker, and R. Sadleir, "In vivo quantification of intraventricular hemorrhage in a neonatal piglet model using an eeg-layout based electrical impedance tomography array," Physiological Measurement, vol. 37, pp. 751-764, 2016.

[45] P. Manwaring, K. Moodie, A. Hartov, K. Manwaring, and R. Halter, "Intracranial electrical impedance tomography: A method of continuous monitoring in an animal model of head trauma," Anesthesia-Analgesia, vol. 117, no. 4, pp. 866875, 2013.

[46] M. Fox and M. Raichle, "Spontaneous fluctuations in brain activity observed with functional magnetic resonance imaging," Nature Reviews Neuroscience, vol. 8, pp. 700-711, 2007.

[47] G. Glover, "Overview of functional magnetic resonance imaging," Neurosurg Clin N Am, vol. 22, no. 2, p. 133-139, 2011.

[48] R. Buxton, E. Wong, and L. Frank, "Dynamics of blood flow and oxygenation changes during brain activation: The balloon model," Magn Reson Med, vol. 39, no. 6, pp. 855-864, 1998. 
[49] S. Sarraf and J. Sun, "Functional brain imaging: A comprehensive survey," arXiv preprint, vol. arXiv:1602.02225, 2016.

[50] G. Morano and J. Seibyl, "Technical overview of brain spect imaging: Improving acquisition and processing of data," J Nucl Med Technol, vol. 31, no. 4, pp. 191$195,2003$.

[51] I. Daly, F. Pichiorri, J. Faller, V. Kaiser, A. Kreilinger, R. Scherer, and G. MüllerPutz, "What does clean eeg look like?," in 2012 Annual International Conference of the IEEE Engineering in Medicine and Biology Society, pp. 3963-3966, 2012.

[52] R. A. Holder DS and H. Y, "Imaging of physiologically evoked responses by electrical impedance tomography with cortical electrodes in the anaesthetised rabbit," Physiological Measurement, vol. 17, pp. A179-186, 1996.

[53] A. Tidswell, A. Gibson, R. Bayford, and D. Holder, "Electrical impedance tomography of human brain activity with a two-dimensional ring of scalp electrodes," Physiological Measurement, vol. 22, no. 1, pp. 167-175, 2001.

[54] T. Tidswell, A. Gibson, R. H. Bayford, and D. S. Holder, "Three-dimensional electrical impedance tomography of human brain activity," NeuroImage, vol. 13, no. 2, pp. $283-294,2001$.

[55] A. P. Bagshaw, A. D. Liston, R. H. Bayford, A. Tizzard, A. P. Gibson, A. Tidswell, M. K. Sparkes, H. Dehghani, C. D. Binnie, and D. S. Holder, "Electrical impedance tomography of human brain function using reconstruction algorithms based on the finite element method," NeuroImage, vol. 20, no. 2, pp. $752-764,2003$. 
[56] R. Gregson, M. Shaw, I. Piper, and R. Clutton, "Transcranial bioimpedance measurement in horses: a pilot study," Veterinary Anaesthesia and Analgesia, vol. 46, no. 5, pp. 620-626, 2019.

[57] A. Tidswell, A. Bagshaw, D. Holder, R. Yerworth, L. Eadie, S. Murray, L. Morgan, and R. Bayford, "A comparison of headnet electrode arrays for electrical impedance tomography of the human head," Physiological Measurement, vol. 24, pp. 527-544, 52003.

[58] S. Xu, M. Dai, and C. Xu, "Performance evaluation of five types of ag/agcl bioelectrodes for cerebral electrical impedance tomography," Annals of Biomedical Engineering, vol. 39, no. 7, pp. 2059-2067, 2011.

[59] J. Sedlacik, Å. Kjørstad, Z. Nagy, J.-H. Buhk, C. R. Behem, C. J. C. Trepte, J. Fiehler, and F. Temme, "Feasibility study of a novel high-flow cold air cooling protocol of the porcine brain using mri temperature mapping.," Therapeutic hypothermia and temperature management, vol. 8 1, pp. 45-52, 2018.

[60] J. Guillen, "Felasa guidelines and recommendations," J Am Assoc Lab Anim Sci., vol. 51, no. 3, pp. 311-321, 2012.

[61] C. Kilkenny, W. Browne, I. Cuthill, M. Emerson, and D. Altman, "Improving bioscience research reporting: The arrive guidelines for reporting animal research," Osteoarthritis Cartilage, vol. 20, no. 4, pp. 256-260, 2012.

[62] M. Jehl, K. Aristovich, M. Faulkner, and D. Holder, "Are patient specific meshes required for eit head imaging?", Physiological Measurement, vol. 37, no. 6, pp. 879-892, 2016. 
[63] A. Adler and W. Lionheart, "Uses and abuses of eidors: an extensible software base for eit," Physiol Meas, vol. 27, pp. S25-S42, 2006.

[64] A. Fieselmann, A. Ganguly, Y. Deuerling-Zheng, M. Zellerhoff, C. Rohkohl, J. Boese, J. Hornegger, and R. Fahrig, "Interventional 4-d c-arm ct perfusion imaging using interleaved scanning and partial reconstruction interpolation," IEEE Transactions on Medical Imaging, vol. 31, no. 4, pp. 892-906, 2012.

[65] R. Merletti, "The electrode-skin interface and optimal detection of bioelectric signals," Physiological Measurement, vol. 31, no. 10, p. E1, 2010.

[66] J. Liu, X. Liu, E. He, F. Gao, Z. Li, G. Xiao, S. Xu, and X. Cai, "A novel drycontact electrode for measuring electroencephalography signals," Sensors and Actuators A: Physical, vol. 294, pp. 73 - 80, 2019.

[67] Y. Huang, J. Dmochowski, Y. Su, A. Datta, C. Rorden, and L. Parra, "Automated mri segmentation for individualized modeling of current flow in the human head," Journal of Neural Engineering, vol. 10, no. 06604, 2013.

[68] A. Owen, K. McMillan, A. Laird, and E. Bullmore, "N-back working memory paradigm: A meta-analysis of normative functional neuroimaging studies," $\mathrm{Hu}$ man Brain Mapping, vol. 25, pp. 46-59, 2005.

[69] E. McAdams, J. Jossinet, A. Lackermeier, and F. Risacher, "Factors affecting electrodegelskin interface impedance in electrical impedance tomography," Med. E3 Biol. Eng. 83 Comput, vol. 34, pp. 397-408, 1996.

[70] B. McDermott, E. Dunne, and O. M, "Haemorrhage detection through svm classification of electrical impedance tomography measurements." https://www.ncbi.nlm.nih.gov/books/NBK549564/, 2019. 2020-09-16. 
[71] A. Adler, "Accounting for erroneous electrode data in electrical impedance tomography," Physiological Measurement, vol. 25, pp. 227-238, 2004.

[72] Y. Mamatjan, P. Gaggero, B. Müller, B. Grychtol, and A. Adler, "Compensating electrode errors due to electrode detachment in electrical impedance tomography," CMBES, vol. 36, no. 1, 2013.

[73] J.-F. P. Abascal, S. R. Arridge, D. Atkinson, R. Horesh, L. Fabrizi, M. D. Lucia], L. Horesh, R. H. Bayford, and D. S. Holder, "Use of anisotropic modelling in electrical impedance tomography; description of method and preliminary assessment of utility in imaging brain function in the adult human head," NeuroImage, vol. 43, no. 2, pp. $258-268,2008$.

[74] A. Burch, "Arrest-related deaths, 2003-2009 - statistical tables," Tech. Rep. NCJ 235385, Bureau of Justice Statistics, 112011.

[75] D. Reay, J. Howard, C. Flinger, and R. Ward, "Effects of positional restraint on saturation and heart rate following exercise," Am J Forensic Med Pathol, vol. 9, no. 1 , pp. $16-18,1988$.

[76] T. Chan, G. Vilke, T. Neuman, and J. Clausen, "Restraint position and positional asphyxia," Ann Emerg Med, vol. 30, no. 5, pp. 578-586, 1997.

[77] P. Schmidt and T. Snowden, "The effects of positional restraint on heart rate and oxygen saturation," J. Emerg. Med., vol. 17, no. 5, pp. 777-782, 1999.

[78] B. Michalewicz, T. Chan, G. Vilke, S. Levy, T. Neuman, and F. Kolkhorst, "Ventilatory and metabolic demands during aggressive physical restraint in healthy adults," Forensic Sci, vol. 52, no. 1, pp. 171-175, 2007. 
[79] C. Sloane, T. Chan, F. Kolkhorst, T. Neuman, E. Castillo, and G. Vilke, "Evaluation of the ventilatory effects of the prone maximum restraint (pmr) position on obese human subjects," Forensic Sci. Int., vol. 237, pp. 86-89, 2014.

[80] S. Karsch, "The problem of police-related cardiac arrest," J Forensic Leg Med, vol. 41, pp. 36-41, 2016.

[81] D. Banks, M. Planty, L. Couzens, P. Lee, C. Brooks, K. Scott, and A. Whyde, "Arrest-related deaths program: pilot study of redesigned survey methodology," Tech. Rep. NCJ 252675, Bureau of Justice Statistics, 72019.

[82] S. Stowe, M. Campbell, J. Dawson, and A. Adler, "Monitoring regional lung volumes during weighted restraint," in Conference EIT 2019 (20th International Conference on Biomedical Applications of Electrical Impedance Tomography, ed.), (London, UK), p. 52, July 1-3 2019.

[83] B. Grychtol, J. Schramel, F. Braun, T. Riedel, U. Auer, M. Mosing, C. B. F. Braun, A. Waldmann, S. Böhm, and A. Adler, "Thoracic eit in 3d - experiences and recommendations," Physiol. Meas., vol. 40, no. 7, p. 074006, 2019.

[84] I. Frerichs, B. Amato, A. van Kaam, D. Tingay, Z. Zhao, B. Grychtol, M. Bodenstein, H. Gagnon, S. Böhm, E. Teschner, O. Stenqvist, T. Mauri, V. Torsani, L. Camporota, A. Schibler, G. Wolf, D. Gommers, S. Leonhardt, and A. Adler, "Chest electrical impedance tomography examination, data analysis, terminology, clinical use and recommendations: consensus statement of the translational eit development study group," Thorax, vol. 0, pp. 1-11, 2016.

[85] G. Teasdale and B. Jennett, "Assessment of coma and impaired consciousness: A practical scale," The Lancet, vol. 304, no. 7872, pp. 81 - 84, 1974. Originally published as Volume 2, Issue 7872. 
[86] B. Grychtol, B. Müller, and A. Adler, "3d eit image reconstruction with greit," Physiol Meas, vol. 37, pp. 785-800, 2016.

[87] V. Sobota and K. Roubik, "Center of ventilation - methods of calculation using electrical impedance tomography and the influence of image segmentation," in IFMBE Proceedings (P. C. e. X. M. C. o. M. Kyriacou E., Christofides S., B. Engineering, and Computing, eds.), vol. 57, pp. 1258-1269, Springer, Cham, 2016.

[88] F. Moreno and H. Lyons, "Effect of body posture on lung volumes," J. Appl. Physiol, vol. 16, no. 1, pp. 27-29, 1961.

[89] T. Coonan and C. Hope, "Cardio-respiratory effects of change of body position," Can Anaesth Soc J, vol. 30, no. 4, pp. 424-437, 1983.

[90] P. Lang, B. Wangelin, M. Bradley, F. Versace, P. Davenport, and V. Costa, "Threat of suffocation and defensive reflex activation," Psychophysiology, vol. 48, no. 3, pp. 393-396, 2011.

[91] M. Bradley, "Natural selective attention: Orienting and emotion," Psychophysiology, vol. 46, no. 1, pp. 1-11, 2009.

[92] T. Tsukamoto, H. Maruyama, M. Kato, M. Uchida, and A. Kubo, "Characteristics of respiratory muscle fatigue upon inhalation resistance with a maximal inspiratory mouth pressure of 50\%," Journal of Physical Therapy Science, vol. 31, no. 4, pp. 318-325, 2019. 